\title{
UNIQUE CONTINUATION FROM A GENERALIZED IMPEDANCE EDGE-CORNER FOR MAXWELL'S SYSTEM AND APPLICATIONS TO INVERSE PROBLEMS
}

\author{
HUAIAN DIAO, HONGYU LIU, LONG ZHANG, AND JUN ZOU
}

\begin{abstract}
We consider the time-harmonic Maxwell system in a domain with a generalized impedance edge-corner, namely the presence of two generalized impedance planes that intersect at an edge. The impedance parameter can be $0, \infty$ or a finite nonidentically vanishing variable function. We establish an accurate relationship between the vanishing order of the solutions to the Maxwell system and the dihedral angle of the edge-corner. In particular, if the angle is irrational, the vanishing order is infinity, i.e. strong unique continuation holds from the edge-corner. The establishment of those new quantitative results involve a highly intricate and subtle algebraic argument. The unique continuation study is strongly motivated by our study of a longstanding inverse electromagnetic scattering problem. As a significant application, we derive several novel unique identifiability results in determining a polyhedral obstacle as well as it surface impedance by a single far-field measurement. We also discuss another potential and interesting application of our result in the inverse scattering theory related to the information encoding.
\end{abstract}

Keywords Maxwell's system, generalized impedance plane, edge-corner, vanishing order, inverse electromagnetic scattering, single far-field measurement

Mathematics Subject Classification (2010): 35P05, 35P25, 35R30, 35Q60

\section{INTRODUCTION}

Let $\Omega$ be an open set in $\mathbb{R}^{3}$, bounded or unbounded, and set

$$
\begin{aligned}
H_{l o c}(\operatorname{curl}, \Omega) & =\left\{\left.U\right|_{B} \in H(\operatorname{curl}, B) ; B \text { is any bounded subdomain of } \Omega\right\}, \\
H(\operatorname{curl}, B) & =\left\{U \in L^{2}(B)^{3} ; \nabla \wedge U \in L^{2}(B)^{3}\right\} .
\end{aligned}
$$

Consider the time-harmonic Maxwell equations for $(\mathbf{E}, \mathbf{H}) \in H_{l o c}(\operatorname{curl}, \Omega) \times H_{l o c}(\operatorname{curl}, \Omega)$ :

$$
\nabla \wedge \mathbf{E}-\mathbf{i} k \mathbf{H}=\mathbf{0}, \quad \nabla \wedge \mathbf{H}+\mathbf{i} k \mathbf{E}=\mathbf{0},
$$

where $\mathbf{i}:=\sqrt{-1}$ and $k \in \mathbb{R}_{+}$. In this paper, we are concerned with the unique continuation property (UCP) of the Maxwell system (1.1) in a particular scenario, which is strongly motivated by our study of a longstanding problem in the inverse electromagnetic scattering theory. In what follows, we first present the mathematical setup for our UCP study.

Let $B_{\rho}(\mathbf{x})$ denote a ball of radius $\rho \in \mathbb{R}_{+}$and centered at $\mathbf{x} \in \mathbb{R}^{3}$. In the sequel, for a set $K \subset \mathbb{R}^{3}, B_{\rho}(K):=\left\{\mathbf{x} ; \mathbf{x} \in B_{\rho}(\mathbf{y})\right.$ for any $\left.\mathbf{y} \in K\right\}$. Let $\Pi_{1}$ and $\Pi_{2}$ be two planes in $\mathbb{R}^{3}$ such that $\Pi_{1} \cap \Pi_{2}=\boldsymbol{L}$, where $\boldsymbol{L}$ is a straight line. We suppose that there exists an open line segment $\boldsymbol{l} \Subset \boldsymbol{L}$ and $\rho \in \mathbb{R}_{+}$such that $B_{\rho}(\boldsymbol{l}) \Subset \Omega$. Let $\mathcal{W}\left(\Pi_{1}, \Pi_{2}\right)$ denote one of the wedge domains formed by $\Pi_{1}$ and $\Pi_{2}$, then $\partial \mathcal{W}\left(\Pi_{1}, \Pi_{2}\right) \cap B_{\rho}(\boldsymbol{l})$ is called an edge-corner associated with $\Pi_{1}$ and $\Pi_{2}$; see Fig. 1 for a schematic illustration. In the sequel, we let $\widetilde{\Pi}_{j}, j=1,2$, denote the two flat faces of the edge-corner lying on $\Pi_{j}$, respectively, and denote it by $\mathcal{E}\left(\widetilde{\Pi}_{1}, \widetilde{\Pi}_{2}, \boldsymbol{l}\right)$. Any $\mathbf{x} \in \boldsymbol{l}$ is said to be an edge-corner point of $\mathcal{E}\left(\widetilde{\Pi}_{1}, \widetilde{\Pi}_{2}, \boldsymbol{l}\right)$. 


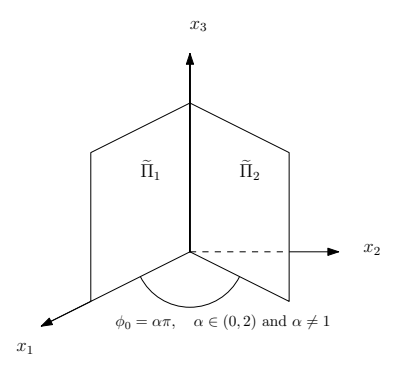

FiguRE 1. Schematic illustration of two intersecting planes with an edgecorner $\mathcal{E}\left(\widetilde{\Pi}_{1}, \widetilde{\Pi}_{2}, \boldsymbol{l}\right)$ and the dihedral angle $\phi_{0}$.

Let $\boldsymbol{\eta}_{j}$ denote a generalized impedance parameter on $\widetilde{\Pi}_{j}$, whose value must fulfil one of the following three possibilities

$$
\text { (i) } \boldsymbol{\eta}_{j} \equiv 0 ; \quad \text { (ii) } \boldsymbol{\eta}_{j} \equiv \infty ; \quad \text { (iii) } \boldsymbol{\eta}_{j} \in L^{\infty}\left(\widetilde{\Pi}_{j}\right) .
$$

Let $\nu_{j} \in \mathbb{S}^{2}$ be the unit normal vector to $\Pi_{j}$, pointing to the exterior of $\mathcal{W}\left(\Pi_{1}, \Pi_{2}\right)$. We introduce the following generalized impedance condition on $\widetilde{\Pi}_{j}$ associated with $(\mathbf{E}, \mathbf{H})$ to the Maxwell system (1.1):

$$
\nu_{j} \wedge(\nabla \wedge \mathbf{E})+\left.\boldsymbol{\eta}_{j}\left(\nu_{j} \wedge \mathbf{E}\right) \wedge \nu_{j}\right|_{\widetilde{\Pi}_{j}}=0 .
$$

In the case $\boldsymbol{\eta}_{j} \equiv \infty,(1.3)$ is understood as

$$
\left.\left(\nu_{j} \wedge \mathbf{E}\right) \wedge \nu_{j}\right|_{\widetilde{\Pi}_{j}}=0 .
$$

An edge-corner $\mathcal{E}\left(\widetilde{\Pi}_{1}, \widetilde{\Pi}_{2}, \boldsymbol{l}\right)$ with the generalized impedance condition 1.3 imposed on $\widetilde{\Pi}_{j}, j=1,2$, is called a generalized impedance edge-corner associated with the Maxwell system (1.1). In this paper, we shall consider the unique continuation property of the solution $(\mathbf{E}, \mathbf{H})$ to 1.1$)$ with the presence of a generalized impedance edge-corner.

The UCP for differential equations from a crack in the domain has been the subject of many existing studies in the literature, see e.g. [2, 8, 9] and the references cited therein. However, the corresponding study to the Maxwell system is rather rare. Moreover, there are several other features that make our current study interestingly new and distinct from many existing UCP studies from cracks. First, the Maxwell system (1.1) is defined in the whole domain $\Omega$, instead of the exterior of the crack, namely $\Omega \backslash \mathcal{E}\left(\Pi_{1}, \Pi_{2}, \boldsymbol{l}\right)$. Usually, for a typical UCP problem from a crack, the differential equation is given over the exterior of the crack, and hence the solution inherits a certain singularity from the pathological geometry of the crack. But in our case, by the standard PDE theory, we know that $(\mathbf{E}, \mathbf{H})$ are real analytic in the interior of $\Omega$, and in particular in $B_{\rho}(\boldsymbol{l})$ which is a neighbourhood of the edge-corner. This makes our UCP study seemingly rather "artificial". However, on the one hand, the UCP problem in this work is strongly motivated by our study of the inverse electromagnetic scattering problems. This shall become more evident in Section 5, and the UCP results shall generate some significant applications that are of both theoretical and practical importance. On the other hand, it turns out that the analyticity of the solutions around the edge-corner is a key factor that helps us to develop an algebraic argument in achieving the desired UCP, though highly intricate and subtle. Second, the edge-corner geometry enables us to establish an accurate relationship between the vanishing order of the solutions to the Maxwell system and the angle of the edgecorner. In particular, if the angle is irrational, then the vanishing order is infinity, i.e. strong unique continuation holds from the edge-corner. We would like to point out that it seems that the extension to the other more general geometry seems rather unpractical, though certain quantitative estimates are more plausible. Third, it is remarked that in 
our UCP study, the Robin-type generalized impedance condition $(1.3)$ is considered on the crack, namely the edge-corner, whereas in most of the existing studies of UCP from cracks, homogeneous Dirichlet-type or Neumann-type conditions are more concerned, which correspond to $\boldsymbol{\eta} \equiv 0$ or $\boldsymbol{\eta} \equiv \infty$, respectively.

As mentioned earlier, we shall consider two interesting and significant applications of the new UCP results to the study of inverse electromagnetic scattering problems. We postpone the mathematical formulation of the inverse problem to Section 6 and we are mainly concerned with the determination of an impenetrable obstacle as well as its boundary impedance by a single electromagnetic far-field measurement. This constitutes a longstanding problem in the inverse scattering theory (cf. [7]). In [11 13, 14], the case $\boldsymbol{\eta} \equiv 0$ or $\boldsymbol{\eta} \equiv \infty$ was considered, and it is shown that a single far-field measurement can uniquely determine an obstacle of the general polyhedral shape and the corresponding stability estimate was established in [12]. The proofs are mainly based on the path argument originated in [16] for the acoustic problem as well as a certain reflection principle for the Maxwell system establish in [13,14. However, the arguments developed therein cannot be extended to tackle the case that the impedance parameter $\boldsymbol{\eta}$ is finite and non-identically zero, even if in the simplest case that it is a finite and nonzero constant, and a fortiori a variable function in our study. Using the UCP results derived in this paper, we are able to establish several novel unique identifiability results for this challenging problem in the polyhedral case, especially in the case that $\boldsymbol{\eta}$ is a finite and non-identically zero variable function. Nevertheless, it is our intention to point out that we shall require certain mild but unobjectionable a-priori knowledge of the underlying polyhedral obstacle as well it surface impedance. The other interesting application of our UCP results is about the "information encoding" for the inverse electromagnetic scattering problems. Indeed, we shall regard our UCP results as generalizing the classical Holmgren's principle [6, 18] for the Maxwell equations. With this view, we can provide an alternative means of electromagnetic scattering measurements for inverse problems that might have some practical implications.

The rest of the paper is organized as follows. Section 2 is devoted to some preliminary knowledge and auxiliary results. In Sections 3 and 4 , we establish the UCP results from a generalized impedance edge-corner for the Maxwell equations (1.1) in two different scenarios. In Section 6, we consider the inverse electromagnetic scattering problems and present two applications of the newly established UCP results.

\section{Preliminaries AND AUXiliary LEMmas}

In this section, we collect some preliminary knowledge for the Maxwell system (1.1) as well as derive several auxiliary lemmas for our subsequent use.

First, we note that the Maxwell system (1.1) is invariant under rigid motions (cf. [3|15]). Hence, throughout the rest of this paper and without loss of generality, we can assume that the edge-corner $\mathcal{E}\left(\widetilde{\Pi}_{1}, \widetilde{\Pi}_{2}, \boldsymbol{l}\right) \Subset \Omega$ satisfies

$$
\boldsymbol{l}=\left\{\mathbf{x}=\left(\mathbf{x}^{\prime}, x_{3}\right) \in \mathbb{R}^{3} ; \mathbf{x}^{\prime}:=\left(x_{1}, x_{2}\right)=\mathbf{0}, x_{3} \in(-h, h)\right\} \Subset \Omega,
$$

where $2 h \in \mathbb{R}_{+}$is the length of $\boldsymbol{l}$, and furthermore $\Pi_{1}$ coincides with the $\left(x_{1}, x_{3}\right)$-plane while $\Pi_{2}$ possesses a dihedral angle $\phi_{0}=\alpha \pi$ away from $\Pi_{1}$ in the anti-clockwise direction; see Fig. 1 for a schematic illustration. Throughout, it is assumed that

$$
\alpha \in(0,2) \text { and } \quad \alpha \neq 1 \text {. }
$$

It can directly verified that the exterior unit normal vectors $\nu_{j}$ to $\Pi_{j}, j=1,2$ are given by

$$
\nu_{1}=(0,-1,0)^{\top}, \quad \nu_{2}=\left(-\sin \phi_{0}, \cos \phi_{0}, 0\right)^{\top}
$$


As specified earlier, we have the generalized impedance condition 1.3 imposed on $\widetilde{\Pi}_{j}$, where the boundary impedance parameter $\boldsymbol{\eta}_{j}$ fulfils 1.2 . In order to consider the unique continuation from the edge-corner as described above, we introduce the following definition.

Definition 2.1. Let $\mathbf{E} \in H_{l o c}(\operatorname{curl}, \Omega)$ be a solution to 1.1 and suppose there exists an edge-corner $\mathcal{E}\left(\widetilde{\Pi}_{1}, \widetilde{\Pi}_{2}, \boldsymbol{l}\right) \Subset \Omega$ as described above. For a given point $\mathbf{x}_{0} \in \boldsymbol{l}$, if there exits a number $N \in \mathbb{N} \cup\{0\}$ such that

$$
\lim _{\rho \rightarrow+0} \frac{1}{\rho^{m}} \int_{B_{\rho}\left(\mathbf{x}_{0}\right)}|\mathbf{E}(\mathbf{x})| \mathrm{d} \mathbf{x}=0 \text { for } m=0,1, \ldots, N+2,
$$

we say that $\mathbf{E}$ vanishes at $\mathbf{x}_{0}$ up to the order $N$. The largest possible $N$ such that (2.3) is fulfilled is called the vanishing order of $\mathbf{E}$ at $\mathbf{x}_{0}$, and we write

$$
\operatorname{Vani}\left(\mathbf{E} ; \mathbf{x}_{0}\right)=N \text {. }
$$

If (2.3) holds for any $N \in \mathbb{N}$, then we say that the vanishing order is infinity.

Since $\mathbf{E}$ is (real) analytic in $\Omega$, we immediately see that if the vanishing order of $\mathbf{E}$ at any point $\mathbf{x}_{0} \in \boldsymbol{l}$ is infinity, then $\mathbf{E} \equiv 0$ in $\Omega$, namely the strong unique continuation property holds. In what follows, it is sufficient to consider the UCP at the origin $\mathbf{0} \in \boldsymbol{l}$. Moreover, due to the symmetry role between $(\mathbf{E}, \mathbf{H})$ and $(-\mathbf{H}, \mathbf{E})$, namely both of them satisfy the same Maxwell system (1.1), we only consider the vanishing order of $\mathbf{E}$, and the same result equally holds for $\mathbf{H}$. It turns out that the vanishing order of $\mathbf{E}$ is related to the rationality of the edge-corner angle, i.e. $\alpha \pi$, and we shall make it more rigorous in the following.

In the subsequent analysis, we shall make frequent use of the spherical coordinate of a point $\mathbf{x}$ in $\mathbb{R}^{3}$ :

$$
\mathbf{x}=(r \sin \theta \cos \phi, r \sin \theta \sin \phi, r \cos \theta):=(r, \theta, \phi), r \geq 0, \theta \in[0, \pi), \phi \in[0,2 \pi) .
$$

It is noted that

$$
\begin{aligned}
& \hat{\boldsymbol{r}}=\sin \theta \cos \phi \cdot \hat{\mathbf{x}}+\sin \theta \sin \phi \cdot \hat{\mathbf{y}}+\cos \theta \cdot \hat{\mathbf{z}} \\
& \hat{\boldsymbol{\theta}}=\cos \theta \cos \phi \cdot \hat{\mathbf{x}}+\cos \theta \sin \phi \cdot \hat{\mathbf{y}}-\sin \theta \cdot \hat{\mathbf{z}} \\
& \hat{\boldsymbol{\phi}}=-\sin \phi \cdot \hat{\mathbf{x}}+\cos \phi \cdot \hat{\mathbf{y}}
\end{aligned}
$$

constitutes an orthonormal basis in the spherical coordinate system, where $\hat{\mathbf{x}}=(1,0,0)^{\top}, \hat{\mathbf{y}}=$ $(0,1,0)^{\top}, \hat{\mathbf{z}}=(0,0,1)^{\top}$.

Definition 2.2. Suppose that $\psi(r, \theta)$ is a complex-valued function for $(r, \theta) \in \Sigma:=\left[0, r_{0}\right] \times$ $\left[-\theta_{0}, \theta_{0}\right]$, where $r_{0}, \theta_{0} \in \mathbb{R}_{+} \cdot \psi$ is said to belong to class $\mathcal{A}$ in $\Sigma$ if it allows an absolutely convergent series representation as follows

$$
\psi(r, \theta)=a_{0}+\sum_{j=1}^{\infty} a_{j}(\theta) r^{j}
$$

where $a_{0} \in \mathbb{C} \backslash\{0\}$ and $a_{j}(\theta) \in C\left[-\theta_{0}, \theta_{0}\right]$.

Two simple scenarios for $\psi(r, \theta)$ to belong to the class $\mathcal{A}$ : first, $\psi$ is a non-zero constant; second, $\psi(r, \theta)$ is real-analytic in $\Sigma$ with $r_{0}, \theta_{0}$ sufficiently small and $\psi(0, \theta)$ independent of $\theta$. For an impedance parameter $\boldsymbol{\eta}_{j}$ in 1.3 in the third case, namely $\boldsymbol{\eta}_{j} \in L^{\infty}\left(\widetilde{\Pi}_{j}\right)$, we readily see that in the $(r, \theta, \phi)$-coordinate, $\left.\phi\right|_{\widetilde{\Pi}_{1}}=0$ and $\left.\phi\right|_{\widetilde{\Pi}_{2}}=\phi_{0}$. In what follows, if for any $\mathbf{x}_{0} \in \boldsymbol{l}$ there exists a neighbourhood $\Sigma_{\mathbf{x}_{0}}$ of $\mathbf{x}_{0}$ which is of the form in Definition 2.2 and is contained in $\widetilde{\widetilde{\Pi}}_{j}$ such that $\psi_{\mathbf{x}_{0}}(r, \theta):=\boldsymbol{\eta}_{j}\left(\mathbf{x}-\mathbf{x}_{0}\right)$ belongs to the class $\mathcal{A}$ in $\Sigma_{\mathbf{x}_{0}}$, then we say that $\eta_{j}$ belongs to the class $\mathcal{A}(\boldsymbol{l})$. It is emphasized that $\boldsymbol{\eta}_{j}$ belonging to the 
class $\mathcal{A}(\boldsymbol{l})$ is a local property, which is localized around a neighbourhood of $\boldsymbol{l}$ on $\widetilde{\Pi}_{j}$. In fact, in our subsequent analysis of the UCP from the edge-corner $\mathcal{E}\left(\widetilde{\Pi}_{1}, \widetilde{\Pi}_{2}, \boldsymbol{l}\right)$ is confined locally around a neighbourhood of $\boldsymbol{l}$, and indeed, around a neighbourhood of the origin $\mathbf{0}$ according to our earlier discussion.

Next, we consider the Fourier representations of the solutions to 1.1 in terms of the spherical waves. Throughout the rest of the paper, for a fixed $l \in \mathbb{N}$ we adopt the notation

$$
[l]_{0}:=\{0, \pm 1, \ldots, \pm l\}, \quad[l]_{1}:=\{ \pm 1, \ldots, \pm l\} .
$$

Recall that the spherical harmonics $Y_{l}^{m}(\theta, \phi)$ is given by

$$
Y_{l}^{m}(\theta, \phi)=c_{l}^{m} P_{l}^{|m|}(\cos \theta) e^{\mathrm{i} m \phi}, \quad c_{l}^{m}=\sqrt{\frac{2 l+1}{4 \pi} \frac{(l-|m|) !}{(l+|m|) !}},
$$

where $P_{l}^{m}(t)$ is the Legendre function. For simplicity, we use the notation $Y_{l}^{m}$ for $Y_{l}^{m}(\theta, \phi)$ from the clear context. For our subsequent use, the following lemma presents some important properties of the associated Legendre functions, which can be conveniently found in [1].

Lemma 2.3. In the spherical coordinate system, the Legendre functions fulfil the following orthogonality condition for any fixed $n \in \mathbb{N}$, and any two integers $m \geq 0$ and $l \leq n$ :

$$
\int_{-\pi}^{\pi} \frac{P_{n}^{m}(\cos \theta) P_{n}^{l}(\cos \theta)}{\sin \theta} d \theta= \begin{cases}0 & \text { if } \quad l \neq m, \\ \frac{(n+m) !}{m(n-m) !} & \text { if } \quad l=m \neq 0 .\end{cases}
$$

Furthermore, the following recursive relationships hold

$$
\begin{aligned}
\frac{\mathrm{d} P_{l}^{|m|}(\cos \theta)}{\mathrm{d} \theta} & =\frac{1}{2}\left[(l+|m|)(l-|m|+1) P_{l}^{|m|-1}(\cos \theta)-P_{l}^{|m|+1}(\cos \theta)\right], \\
\frac{|m|}{\sin \theta} P_{l}^{|m|}(\cos \theta) & =-\frac{1}{2}\left[P_{l-1}^{|m|+1}(\cos \theta)+(l+|m|-1)(l+|m|) P_{l-1}^{|m|-1}(\cos \theta)\right],
\end{aligned}
$$

where $l \in \mathbb{N}$ and $m \in[l]_{0}$. If $P_{l}^{m}(\cos \theta)$ is evaluated at $\theta=0$, for $l \in \mathbb{N} \cup\{0\}$ we have

$$
P_{l}^{m}(1)=0, \quad m \in[l]_{1} ; \quad P_{l}^{0}(1)=1 .
$$

For a fixed $n \in \mathbb{N} \cup\{0\}$ and $m \in \mathbb{N}$ with $m \leq n$, it holds that

$$
P_{n}^{-m}(\cos \theta)=(-1)^{m} \frac{(n-m) !}{(n+m) !} P_{n}^{m}(\cos \theta) .
$$

Recall that the spherical Bessel function $j_{\ell}(t)$ of the order $\ell$ is defined by

$$
j_{\ell}(t)=\frac{t^{\ell}}{(2 \ell+1) ! !}\left(1-\sum_{l=1}^{\infty} \frac{(-1)^{l} t^{2 l}}{2^{l} l !(2 \ell+3) \cdots(2 \ell+2 l+1)}\right)=\frac{t^{\ell}}{(2 \ell+1) ! !}+\mathcal{O}\left(t^{\ell+2}\right) .
$$

There holds the following recursive relationships [1]:

$$
\frac{j_{\ell}(t)}{t}=\frac{j_{\ell-1}(t)+j_{\ell+1}(t)}{2 \ell+1}, \quad j_{\ell}^{\prime}(t)=\frac{\ell j_{\ell-1}(t)-(\ell+1) j_{\ell+1}(t)}{2 \ell+1}, \quad \ell \in \mathbb{N} .
$$

Lemma 2.4. [5, Lemma 2.5] Suppose that for $t \in(0, h), h \in \mathbb{R}_{+}$,

$$
\sum_{n=0}^{\infty} \alpha_{n} j_{n}(t)=0
$$

where $j_{n}(t)$ is the $n$-th spherical Bessel function. Then $\alpha_{n}=0, \quad n=0,1,2, \ldots$ 
Lemma 2.5. [6] Recall that $\hat{\boldsymbol{r}}, \hat{\boldsymbol{\theta}}$ and $\hat{\boldsymbol{\phi}}$ are defined in 2.5$]$. Denote

$$
\mathbf{M}_{l}^{m}(\mathbf{x})=j_{l}(k r) \cdot \mathbf{X}_{l}^{m}, \quad \mathbf{N}_{l}^{m}(\mathbf{x})=\mathbf{i}\left(\frac{j_{l}(k r)}{k r}+j_{l}^{\prime}(k r)\right) \mathbf{Z}_{l}^{m}-\frac{\sqrt{l(l+1)}}{k r} \cdot j_{l}(k r) Y_{l}^{m} \cdot \hat{\boldsymbol{r}},
$$

where $k \in \mathbb{R}_{+}, j_{l}^{\prime}(k r)$ is the derivative of $j_{l}(k r)$ with respect to $k r$, and

$$
\mathbf{X}_{l}^{m}=\frac{\mathbf{i}}{\sqrt{l(l+1)}}\left(\frac{\mathbf{i} \cdot m}{\sin \theta} Y_{l}^{m} \hat{\boldsymbol{\theta}}-\frac{\partial Y_{l}^{m}}{\partial \theta} \cdot \hat{\boldsymbol{\phi}}\right), \quad \mathbf{Z}_{l}^{m}=\frac{\mathbf{i}}{\sqrt{l(l+1)}}\left(\frac{\partial Y_{l}^{m}}{\partial \theta} \hat{\boldsymbol{\theta}}+\frac{\mathbf{i} \cdot m}{\sin \theta} Y_{l}^{m} \hat{\boldsymbol{\phi}}\right) .
$$

The solution $\mathbf{E}(\mathbf{x})$ to (1.1) has the following Fourier expansion around $\mathbf{0}$,

$$
\mathbf{E}(\mathbf{x})=\sum_{l=1}^{\infty} \sum_{m=-l}^{l}\left(a_{l}^{m} \cdot \mathbf{M}_{l}^{m}(\mathbf{x})+b_{l}^{m} \cdot \mathbf{N}_{l}^{m}(\mathbf{x})\right), \quad a_{l}^{m}, b_{l}^{m} \in \mathbb{C},
$$

which (together with its derivatives) converges uniformly in $B_{\rho_{0}}(\mathbf{0})$ for a sufficiently small $\rho_{0} \in \mathbb{R}_{+}$.

Using (2.14), from Lemma 2.5, we can derive that

$$
\begin{aligned}
\mathbf{E}(\mathbf{x})= & -\sum_{l=1}^{\infty} \sum_{m=-l}^{l} \frac{1}{\sqrt{l(l+1)}}\left\{b_{l}^{m} \cdot l(l+1) p_{l}(k r) \cdot Y_{l}^{m} \cdot \hat{\boldsymbol{r}}\right. \\
& +\left[a_{l}^{m} \cdot j_{l}(k r) \frac{m}{\sin \theta} Y_{l}^{m}+b_{l}^{m} \cdot q_{l}(k r) \cdot \frac{\partial Y_{l}^{m}}{\partial \theta}\right] \cdot \hat{\boldsymbol{\theta}} \\
& \left.+\mathbf{i}\left[a_{l}^{m} \cdot j_{l}(k r) \frac{\partial Y_{l}^{m}}{\partial \theta}+b_{l}^{m} \cdot q_{l}(k r) \frac{m}{\sin \theta} Y_{l}^{m}\right] \cdot \hat{\boldsymbol{\phi}}\right\},
\end{aligned}
$$

where

$$
p_{l}(k r)=\frac{j_{l-1}(k r)+j_{l+1}(k r)}{2 l+1}, \quad q_{l}(k r)=\frac{(l+1) j_{l-1}(k r)-l j_{l+1}(k r)}{2 l+1} .
$$

Remark 2.6. In view of (2.13), the lowest order terms of $p_{l}(k r)$ and $q_{l}(k r)$ with respect to the power of $r$ are

$$
\frac{k^{l-1}}{(2 l+1)(2 l-1) ! !} r^{l-1} \text { and } \frac{(l+1) k^{l-1}}{(2 l+1)(2 l-1) ! !} r^{l-1}
$$

respectively.

Lemma 2.7. [10, Proposition 2.1.7] If the power series $\sum_{\mu} a_{\mu} \mathrm{x}^{\mu}$ converges at a point $\mathbf{x}_{0}$, then it converges uniformly and absolutely on compact subsets of $U\left(\mathbf{x}_{\mathbf{0}}\right)$, where

$U\left(\mathbf{x}_{0}\right)=\left\{\left(r_{1} x_{0,1}, \ldots, r_{n} x_{0, n}\right):-1<r_{j}<1, j=1, \ldots, n\right\}, \mathbf{x}_{0}=\left(x_{0,1}, \ldots, x_{0, n}\right) \in \mathbb{R}^{n}$.

Using Definition 2.1, in view of (2.17), we can obtain the following lemma.

Lemma 2.8. Let $\mathbf{E}$ be a solution to (1.1). Recall that $\mathbf{E}$ has the radial wave expansion (2.17) in $B_{\rho_{0}}(\mathbf{0})$. For a fixed $N \in \mathbb{N}$, if

$$
a_{l}^{m}=b_{l}^{m}=0, \quad m \in[l]_{0}, \quad l=1,2, \ldots, N,
$$

where $[l]_{0}$ is defined in (2.7), then

$$
\operatorname{Vani}(\mathbf{E} ; \mathbf{0}) \geq N \text {. }
$$

Conversely, if there exits $N \in \mathbb{N}$ such that 2.20) holds then we have 2.19. 
Proof. From Lemma 2.7, we know that 2.17) converges uniformly and absolutely in $B_{\rho_{1}}(\mathbf{0})$, where $0<\rho_{1}<\rho_{0}$. Substituting (2.19) into (2.17), we have

$$
\begin{aligned}
\mathbf{E}(\mathbf{x})= & -\sum_{l=N+1}^{\infty} \sum_{m=-l}^{l} \frac{1}{\sqrt{l(l+1)}}\left\{b_{l}^{m} \cdot l(l+1) p_{l}(k r) \cdot Y_{l}^{m} \cdot \hat{\boldsymbol{r}}\right. \\
& +\left[a_{l}^{m} \cdot j_{l}(k r) \frac{m}{\sin \theta} Y_{l}^{m}+b_{l}^{m} \cdot q_{l}(k r) \cdot \frac{\partial Y_{l}^{m}}{\partial \theta}\right] \cdot \hat{\boldsymbol{\theta}} \\
& \left.+\mathbf{i}\left[a_{l}^{m} \cdot j_{l}(k r) \frac{\partial Y_{l}^{m}}{\partial \theta}+b_{l}^{m} \cdot q_{l}(k r) \frac{m}{\sin \theta} Y_{l}^{m}\right] \cdot \hat{\boldsymbol{\phi}}\right\} .
\end{aligned}
$$

From Remark 2.6, the lowest order of $r$ with respect to the power of $r$ in 2.22$)$ is $N$. Therefore,

$$
\begin{aligned}
\frac{\mathbf{E}(\mathbf{x})}{r^{N}}= & -\sum_{l=N+1}^{\infty} \sum_{m=-l}^{l} \frac{1}{\sqrt{l(l+1)}}\left\{b_{l}^{m} \cdot l(l+1) \frac{p_{l}(k r)}{r^{N}} \cdot Y_{l}^{m} \cdot \hat{\boldsymbol{r}}\right. \\
& +\left[a_{l}^{m} \cdot \frac{j_{l}(k r)}{r^{N}} \frac{m}{\sin \theta} Y_{l}^{m}+b_{l}^{m} \cdot \frac{q_{l}(k r)}{r^{N}} \cdot \frac{\partial Y_{l}^{m}}{\partial \theta}\right] \cdot \hat{\boldsymbol{\theta}} \\
& \left.+\mathbf{i}\left[a_{l}^{m} \cdot \frac{j_{l}(k r)}{r^{N}} \frac{\partial Y_{l}^{m}}{\partial \theta}+b_{l}^{m} \cdot \frac{q_{l}(k r)}{r^{N}} \frac{m}{\sin \theta} Y_{l}^{m}\right] \cdot \hat{\boldsymbol{\phi}}\right\}
\end{aligned}
$$

converges uniformly and absolutely in $B_{\rho_{1}}(\mathbf{0})$, which implies

$$
\left|\frac{\mathbf{E}(\mathbf{x})}{r^{N}}\right|=\mathcal{O}(1), \quad \text { as } r \rightarrow+0 .
$$

In view of Definition 2.1, by virtue of 2.23 , we have

$$
\lim _{\rho \rightarrow+0} \frac{1}{\rho^{m}} \int_{B_{\rho}(\mathbf{0})}|\mathbf{E}(\mathbf{x})| \mathrm{d} \mathbf{x} \leq \lim _{\rho \rightarrow+0} \frac{\rho^{N+2}}{\rho^{m}} \int_{0}^{\rho} \int_{0}^{\pi} \int_{0}^{2 \pi}\left|\frac{\mathbf{E}(\mathbf{x})}{r^{N}}\right| \mathrm{d} r \mathrm{~d} \theta \mathrm{d} \phi=0,
$$

which holds for $m=0,1, \ldots, N+2$, and this proves $(2.20)$. The other direction of the conclusion can be proved by using similar arguments.

Lemma 2.9. Let $\mathbf{E}$ be a solution to (1.1). Recall that $\mathbf{E}$ has the radial wave expansion (2.17) in $B_{\rho_{0}}(\mathbf{0})$. Consider an edge-corner $\mathcal{E}\left(\widetilde{\Pi}_{1}, \widetilde{\Pi}_{2}, \boldsymbol{l}\right) \Subset \Omega$ associated with $\mathbf{E}$. Recall that $\nu_{i}$ defined in (2.2) are the outward unit normal vectors to $\Pi_{i}, i=1,2$. Then

$$
\begin{aligned}
\left.\nu_{1} \wedge \mathbf{E}\right|_{\widetilde{\Pi}_{1}}= & \sum_{l=1}^{\infty} \sum_{m=-l}^{l}-\frac{1}{\sqrt{l(l+1)}}\left\{\left.b_{l}^{m} l(l+1) p_{l}(k r) Y_{l}^{m}\right|_{\phi=0} \boldsymbol{e}_{\mathbf{1}}(\theta, 0)\right. \\
& \left.+\left(\left.a_{l}^{m} j_{l}(k r) \frac{m}{\sin \theta} Y_{l}^{m}\right|_{\phi=0}+\left.b_{l}^{m} \cdot q_{l}(k r) \frac{\partial Y_{l}^{m}}{\partial \theta}\right|_{\phi=0}\right) \boldsymbol{e}_{\mathbf{2}}(\theta, 0)\right\} \\
\left.\nu_{2} \wedge \mathbf{E}\right|_{\widetilde{\Pi}_{2}}= & \sum_{l=1}^{\infty} \sum_{m=-l}^{l}-\frac{1}{\sqrt{l(l+1)}}\left\{\left.b_{l}^{m} l(l+1) p_{l}(k r) Y_{l}^{m}\right|_{\phi=\phi_{0}} \boldsymbol{e}_{\mathbf{1}}\left(\theta, \phi_{0}\right)\right. \\
& \left.+\left(\left.a_{l}^{m} j_{l}(k r) \frac{m}{\sin \theta} Y_{l}^{m}\right|_{\phi=\phi_{0}}+\left.b_{l}^{m} \cdot q_{l}(k r) \frac{\partial Y_{l}^{m}}{\partial \theta}\right|_{\phi=\phi_{0}}\right) \boldsymbol{e}_{\mathbf{2}}\left(\theta, \phi_{0}\right)\right\}
\end{aligned}
$$

where

$$
\boldsymbol{e}_{1}(\theta, \phi)=\left[\begin{array}{c}
\cos \phi \cos \theta \\
\sin \phi \cos \theta \\
-\sin \theta
\end{array}\right] \text { and } \boldsymbol{e}_{2}(\theta, \phi)=-\left[\begin{array}{c}
\cos \phi \sin \theta \\
\sin \phi \sin \theta \\
\cos \theta
\end{array}\right]
$$


are linearly independent for any $\theta$ and $\phi$. Furthermore, we have

$$
\begin{aligned}
\nu_{1} \wedge\left(\left.\nabla \wedge \mathbf{E}\right|_{\widetilde{\Pi}_{1}}\right)= & \mathbf{i} k \sum_{l=1}^{\infty} \sum_{m=-l}^{l} \frac{1}{\sqrt{l(l+1)}}\left\{\left.a_{l}^{m} l(l+1) p_{l}(k r) Y_{l}^{m}\right|_{\phi=0} \cdot \boldsymbol{e}_{\mathbf{1}}(\theta, 0)\right. \\
& \left.+\left(-b_{l}^{m} j_{l}(k r) \cdot \frac{m}{\sin \theta} Y_{l}^{m}+\left.a_{l}^{m} \cdot q_{l}(k r) \cdot \frac{\partial Y_{l}^{m}}{\partial \theta}\right|_{\phi=0}\right) \cdot \boldsymbol{e}_{\mathbf{2}}(\theta, 0)\right\}, \\
\nu_{2} \wedge\left(\left.\nabla \wedge \mathbf{E}\right|_{\widetilde{\Pi}_{2}}\right)= & \mathbf{i} k \sum_{l=1}^{\infty} \sum_{m=-l}^{l} \frac{1}{\sqrt{l(l+1)}}\left\{\left.a_{l}^{m} l(l+1) p_{l}(k r) Y_{l}^{m}\right|_{\phi=\phi_{0}} \cdot \boldsymbol{e}_{\mathbf{1}}\left(\theta, \phi_{0}\right)\right. \\
& \left.+\left(-\left.b_{l}^{m} j_{l}(k r) \cdot \frac{m}{\sin \theta} Y_{l}^{m}\right|_{\phi=\phi_{0}}+\left.a_{l}^{m} q_{l}(k r) \cdot \frac{\partial Y_{l}^{m}}{\partial \theta}\right|_{\phi=\phi_{0}}\right) \cdot \boldsymbol{e}_{\mathbf{2}}\left(\theta, \phi_{0}\right)\right\} .
\end{aligned}
$$

Proof. Using the fact that $\phi=\phi_{0}$ for $\mathbf{x}=(r, \theta, \phi) \in \Pi_{2}$, it is easy to see that $\nu_{2} \wedge\left(\left.\hat{\boldsymbol{r}}\right|_{\phi=\phi_{0}}\right)=\left[\begin{array}{c}\cos \phi_{0} \cos \theta \\ \sin \phi_{0} \sin \theta \\ -\sin \theta\end{array}\right], \quad \nu_{2} \wedge\left(\left.\hat{\boldsymbol{\theta}}\right|_{\phi=\phi_{0}}\right)=\left[\begin{array}{c}-\cos \phi_{0} \sin \theta \\ -\sin \phi_{0} \sin \theta \\ -\cos \theta\end{array}\right], \quad \nu_{2} \wedge\left(\left.\hat{\boldsymbol{\phi}}\right|_{\phi=\phi_{0}}\right)=\mathbf{0}$,

from which we can derive the second equation of (2.24). The first equation of (2.24) can be obtained in a similar way.

Recall that $\mathbf{M}_{l}^{m}(\mathbf{x})$ and $\mathbf{N}_{l}^{m}(\mathbf{x})$ are defined in 2.16$)$. Using the identity $\nabla \wedge \mathbf{M}_{l}^{m}(\mathbf{x})=$ $-\mathbf{i} k \mathbf{N}_{l}^{m}(\mathbf{x})$ and $\nabla \wedge \mathbf{N}_{l}^{m}(\mathbf{x})=\mathbf{i} k \mathbf{M}_{l}^{m}(\mathbf{x})($ cf. 6]) we can obtain that

$$
\begin{aligned}
\left.\nabla \wedge \mathbf{E}\right|_{\widetilde{\Pi}_{1}}=\mathbf{i} k \sum_{l=1}^{\infty} \sum_{m=-l}^{l} & \frac{1}{\sqrt{l(l+1)}}\left\{\left.a_{l}^{m} \cdot l(l+1) p_{l}(k r) Y_{l}^{m} \cdot \nu_{1} \wedge \hat{\boldsymbol{r}}\right|_{\phi=0}\right. \\
& +\left.\left(-b_{l}^{m} j_{l}(k r) \frac{m}{\sin \theta} Y_{l}^{m}+a_{l}^{m} q_{l}(k r) \frac{\partial Y_{l}^{m}}{\partial \theta}\right) \cdot \nu_{1} \wedge \hat{\boldsymbol{\theta}}\right|_{\phi=0} \\
& \left.+\left.\left(-b_{l}^{m} j_{l}(k r) \mathbf{i} \frac{\partial Y_{l}^{m}}{\partial \theta}+a_{l}^{m} \cdot q_{l}(k r) \frac{\mathbf{i} m}{\sin \theta} Y_{l}^{m}\right) \cdot \nu_{1} \wedge \hat{\boldsymbol{\phi}}\right|_{\phi=0}\right\}, \\
\left.\nabla \wedge \mathbf{E}\right|_{\widetilde{\Pi}_{2}}=\mathbf{i} k \sum_{l=1}^{\infty} \sum_{m=-l}^{l} & \frac{1}{\sqrt{l(l+1)}}\left\{\left.a_{l}^{m} \cdot l(l+1) p_{l}(k r) Y_{l}^{m} \cdot \nu_{2} \wedge \hat{\boldsymbol{r}}\right|_{\phi=\phi_{0}}\right. \\
& +\left.\left(-b_{l}^{m} j_{l}(k r) \frac{m}{\sin \theta} Y_{l}^{m}+a_{l}^{m} q_{l}(k r) \frac{\partial Y_{l}^{m}}{\partial \theta}\right) \nu_{2} \wedge \hat{\boldsymbol{\theta}}\right|_{\phi=\phi_{0}} \\
& \left.+\left.\left(-b_{l}^{m} j_{l}(k r) \mathbf{i} \frac{\partial Y_{l}^{m}}{\partial \theta}+a_{l}^{m} \cdot q_{l}(k r) \frac{\mathbf{i} m}{\sin \theta} Y_{l}^{m}\right) \nu_{2} \wedge \hat{\boldsymbol{\phi}}\right|_{\phi=\phi_{0}}\right\} .
\end{aligned}
$$

Combing (2.28) with (2.27), together with straightforward though a bit tedious calculations, one can deduce the second equation of (2.26). The first equation of 2.26 ) can be shown in a similar manner.

The proof is complete.

Lemma 2.10. Let $\mathbf{E}$ be a solution to 1.1$)$. Recall that $\mathbf{E}$ has the radial wave expansion 2.17 in $B_{\rho_{0}}(\mathbf{0})$. Consider an edge-corner $\mathcal{E}\left(\widetilde{\Pi}_{1}, \widetilde{\Pi}_{2}, \boldsymbol{l}\right) \Subset \Omega$ associated with $\mathbf{E}$. Recall that $\nu_{i}$ defined in 2.2 are the outward unit normal vectors to $\Pi_{i}, i=1,2$. Assume that $\boldsymbol{\eta}_{1}, \boldsymbol{\eta}_{2}$ 
belong to the class $\mathcal{A}(\boldsymbol{l})$. Then we have

$$
\begin{aligned}
& \nu_{1} \wedge\left(\left.\nabla \wedge \mathbf{E}\right|_{\widetilde{\Pi}_{1}}\right)+\boldsymbol{\eta}_{1}\left(\left.\nu_{1} \wedge \mathbf{E}\right|_{\widetilde{\Pi}_{1}}\right) \wedge \nu_{1} \\
= & \sum_{l=1}^{\infty} \sum_{m=-l}^{l} \frac{1}{\sqrt{l(l+1)}}\left\{\left(\mathbf{i} k a_{l}^{m} l(l+1) p_{l}(k r) Y_{l}^{m}-\boldsymbol{\eta}_{1} a_{l}^{m} j_{l}(k r) \frac{m}{\sin \theta} Y_{l}^{m}\right.\right. \\
& \left.-\boldsymbol{\eta}_{1} b_{l}^{m} q_{l}(k r) \frac{\partial Y_{l}^{m}}{\partial \theta}\right) \boldsymbol{e}_{\mathbf{1}}(\theta, 0)+\left(-\mathbf{i} k b_{l}^{m} j_{l}(k r) \frac{m}{\sin \theta} Y_{l}^{m}+\mathbf{i} k a_{l}^{m} q_{l}(k r) \frac{\partial Y_{l}^{m}}{\partial \theta}\right. \\
& \left.\left.+\boldsymbol{\eta}_{1} b_{l}^{m} l(l+1) p_{l}(k r) Y_{l}^{m}\right) \cdot \boldsymbol{e}_{\mathbf{2}}(\theta, 0)\right\} \\
& \nu_{1} \wedge\left(\left.\nabla \wedge \mathbf{E}\right|_{\widetilde{\Pi}_{1}}\right)+\boldsymbol{\eta}_{1}\left(\left.\nu_{1} \wedge \mathbf{E}\right|_{\widetilde{\Pi}_{1}}\right) \wedge \nu_{1} \\
= & \sum_{l=1}^{\infty} \sum_{m=-l}^{l} \frac{1}{\sqrt{l(l+1)}}\left\{\left(\mathbf{i} k a_{l}^{m} l(l+1) p_{l}(k r) Y_{l}^{m}-\boldsymbol{\eta}_{1} a_{l}^{m} j_{l}(k r) \frac{m}{\sin \theta} Y_{l}^{m}\right.\right. \\
& \left.-\boldsymbol{\eta}_{1} b_{l}^{m} q_{l}(k r) \frac{\partial Y_{l}^{m}}{\partial \theta}\right) \boldsymbol{e}_{\mathbf{1}}(\theta, 0)+\left(-\mathbf{i} k b_{l}^{m} j_{l}(k r) \frac{m}{\sin \theta} Y_{l}^{m}+\mathbf{i} k a_{l}^{m} q_{l}(k r) \frac{\partial Y_{l}^{m}}{\partial \theta}\right. \\
& \left.\left.+\boldsymbol{\eta}_{1} b_{l}^{m} l(l+1) p_{l}(k r) Y_{l}^{m}\right) \cdot \boldsymbol{e}_{\mathbf{2}}(\theta, 0)\right\},
\end{aligned}
$$

and

$$
\begin{aligned}
& \nu_{2} \wedge\left(\left.\nabla \wedge \mathbf{E}\right|_{\widetilde{\Pi}_{2}}\right)+\boldsymbol{\eta}_{2}\left(\left.\nu_{2} \wedge \mathbf{E}\right|_{\widetilde{\Pi}_{2}}\right) \wedge \nu_{2} \\
= & \sum_{l=1}^{\infty} \sum_{m=-l}^{l} \frac{1}{\sqrt{l(l+1)}}\left\{\left(\mathbf{i} k a_{l}^{m} l(l+1) p_{l}(k r) Y_{l}^{m}-\boldsymbol{\eta}_{2} a_{l}^{m} j_{l}(k r) \frac{m}{\sin \theta} Y_{l}^{m}\right.\right. \\
& \left.-\boldsymbol{\eta}_{2} b_{l}^{m} q_{l}(k r) \frac{\partial Y_{l}^{m}}{\partial \theta}\right) \boldsymbol{e}_{\mathbf{1}}\left(\theta, \phi_{0}\right)+\left(-\mathbf{i} k b_{l}^{m} j_{l}(k r) \frac{m}{\sin \theta} Y_{l}^{m}+\mathbf{i} k a_{l}^{m} q_{l}(k r) \frac{\partial Y_{l}^{m}}{\partial \theta}\right. \\
& \left.\left.+\boldsymbol{\eta}_{2} b_{l}^{m} l(l+1) p_{l}(k r) Y_{l}^{m}\right) \cdot \boldsymbol{e}_{\mathbf{2}}\left(\theta, \phi_{0}\right)\right\},
\end{aligned}
$$

where $\boldsymbol{e}_{\mathbf{1}}(\theta, 0), \boldsymbol{e}_{\mathbf{2}}(\theta, 0) \boldsymbol{e}_{\mathbf{1}}\left(\theta, \phi_{0}\right)$ and $\boldsymbol{e}_{\mathbf{2}}\left(\theta, \phi_{0}\right)$ are defined in $(2.25)$.

Proof. Recall that $\nu_{2}$ is defined in $(2.2), \hat{\boldsymbol{r}}, \hat{\boldsymbol{\theta}}$ and $\hat{\boldsymbol{\phi}}$ are given by 2.5$)$. Then it is easy to see that

$$
\begin{aligned}
& \left(\nu_{2} \wedge \hat{\boldsymbol{r}}\right) \wedge \nu_{2}=\left(\cos \phi_{0} \sin \theta, \sin \phi_{0} \sin \theta, \cos \theta\right)^{\top}, \\
& \left(\nu_{2} \wedge \hat{\boldsymbol{\theta}}\right) \wedge \nu_{2}=\left(\cos \phi_{0} \cos \theta, \sin \phi_{0} \cos \theta,-\sin \theta\right)^{\top}, \quad\left(\nu_{2} \wedge \hat{\boldsymbol{\phi}}\right) \wedge \nu_{2}=\mathbf{0} .
\end{aligned}
$$

Using (2.17) and (2.26), we can derive that

$$
\begin{aligned}
& \nu_{2} \wedge\left(\left.\nabla \wedge \mathbf{E}\right|_{\widetilde{\Pi}_{2}}\right)+\boldsymbol{\eta}_{2}\left(\left.\nu_{2} \wedge \mathbf{E}\right|_{\widetilde{\Pi}_{2}}\right) \wedge \nu_{2} \\
= & \sum_{l=1}^{\infty} \sum_{m=-l}^{l} \frac{1}{\sqrt{l(l+1)}}\left\{\mathbf { i } k \left(a_{l}^{m} l(l+1) p_{l}(k r) Y_{l}^{m} \cdot \nu_{2} \wedge \hat{\boldsymbol{r}}\right.\right. \\
& +\left(-b_{l}^{m} j_{l}(k r) \cdot \frac{m}{\sin \theta} Y_{l}^{m}+a_{l}^{m} q_{l}(k r) \frac{\partial Y_{l}^{m}}{\partial \theta}\right) \cdot \nu_{2} \wedge \hat{\boldsymbol{\theta}} \\
& \left.+\left(-b_{l}^{m} j_{l}(k r) \mathbf{i} \frac{\partial Y_{l}^{m}}{\partial \theta}+a_{l}^{m} q_{l}(k r) \frac{\mathbf{i} m}{\sin \theta} Y_{l}^{m}\right) \nu_{2} \wedge \hat{\boldsymbol{\phi}}\right)
\end{aligned}
$$




$$
\begin{aligned}
& -\boldsymbol{\eta}_{2}\left(\left(b_{l}^{m} \cdot l(l+1) p_{l}(k r) \cdot Y_{l}^{m}\right) \cdot\left(\nu_{2} \wedge \hat{\mathbf{r}}\right) \wedge \nu_{2}\right. \\
& +\left(a_{l}^{m} j_{l}(k r) \frac{m}{\sin \theta} Y_{l}^{m}+b_{l}^{m} q_{l}(k r) \frac{\partial Y_{l}^{m}}{\partial \theta}\right)\left(\nu_{2} \wedge \hat{\boldsymbol{\theta}}\right) \wedge \nu_{2} \\
& \left.\left.+\mathbf{i}\left(a_{l}^{m} j_{l}(k r) \frac{\partial Y_{l}^{m}}{\partial \theta}+\frac{m b_{l}^{m} q_{l}(k r)}{\sin \theta} Y_{l}^{m}\right)\left(\nu_{2} \wedge \hat{\boldsymbol{\phi}}\right) \wedge \nu_{2}\right)\right\} .
\end{aligned}
$$

Substituting (2.27) and (2.32) into (2.33), together with straightforward calculations, we can obtain (2.31). 2.30 can be derived in a similar manner.

\section{VANiShing ORDERS FOR AN EDGE-CORNER $\mathcal{E}\left(\widetilde{\Pi}_{1}, \widetilde{\Pi}_{2}, \boldsymbol{l}\right)$ WITH $\boldsymbol{\eta}_{j} \in \mathcal{A}(\boldsymbol{l})$}

In this section, we consider the case that $\mathcal{E}\left(\widetilde{\Pi}_{1}, \widetilde{\Pi}_{2}, \boldsymbol{l}\right)$ and edge-corner with both $\boldsymbol{\eta}_{1}$ and $\boldsymbol{\eta}_{2}$ belong to the class $\mathcal{A}(\boldsymbol{l})$. We shall derive the vanishing order of $\mathbf{E}$ to $(1.1)$ at the origin $\mathbf{0} \in \boldsymbol{l}$. The major idea is to make use of the radial wave expansion (2.17) of $\mathbf{E}$ in $B_{\rho_{0}}(\mathbf{0})$, and to investigate the relationships between $a_{n}^{ \pm 1}, a_{n}^{0}$ and $b_{n}^{ \pm 1}, b_{n}^{0}$. Henceforth, according to Definition 2.2. we assume that $\boldsymbol{\eta}_{j}, j=1,2$, are given by the following absolutely convergent series at $\mathbf{0} \in \boldsymbol{l}$ :

$$
\begin{aligned}
& \boldsymbol{\eta}_{1}=\eta_{1}+\sum_{j=1}^{\infty} \eta_{1, j}(\theta) r^{j} \\
& \boldsymbol{\eta}_{2}=\eta_{2}+\sum_{j=1}^{\infty} \eta_{2, j}(\theta) r^{j}
\end{aligned}
$$

where $\eta_{\ell} \in \mathbb{C} \backslash\{0\}, \eta_{\ell, j}(\theta) \in C[-\pi, \pi]$ and $r \in[-h, h], \ell=1,2$. Next, based on the above setting, we derive several critical lemmas.

Lemma 3.1. Let $\mathbf{E}$ be a a solution to (1.1), whose radial wave expansion in $B_{\rho_{0}}(\mathbf{0})$ is given by 2.17). Consider a generalized impedance edge-corner $\mathcal{E}\left(\widetilde{\Pi}_{1}, \widetilde{\Pi}_{2}, \boldsymbol{l}\right) \Subset \Omega$ with $\angle\left(\Pi_{1}, \Pi_{2}\right)=\phi_{0}=\alpha \pi$, where $\alpha \in(0,2)$ and $\alpha \neq 1$. Suppose that the generalized impedance parameters $\boldsymbol{\eta}_{j}$ on $\widetilde{\Pi}_{j}, j=1,2$, are given by 3.1a) and $3.1 \mathrm{~b}$ respectively. It holds that

$$
\begin{aligned}
& 0=\frac{4 \mathbf{i} k c_{1}^{1} \sin ^{2} \phi_{0}}{6 \sqrt{2}}\left(a_{1}^{1}+a_{1}^{-1}\right)-\frac{4 k c_{1}^{1} \sin \phi_{0} \cos \phi_{0}}{6 \sqrt{2}}\left(a_{1}^{1}-a_{1}^{-1}\right)-\frac{\left(\eta_{2} \cos \phi_{0}+\eta_{1}\right) \sqrt{2} c_{1}^{0}}{3} b_{1}^{0}, \\
& 0=-\frac{4 \mathbf{i} k c_{1}^{1} \sin \phi_{0} \cos \phi_{0}}{6 \sqrt{2}}\left(a_{1}^{1}+a_{1}^{-1}\right)-\frac{4 k c_{1}^{1} \sin ^{2} \phi_{0}}{6 \sqrt{2}}\left(a_{1}^{1}-a_{1}^{-1}\right)-\frac{\eta_{2} \sqrt{2} c_{1}^{0} \sin \phi_{0}}{3} b_{1}^{0}, \\
& 0=-\frac{4 c_{1}^{1}\left(-\eta_{1}+\eta_{2} \cos \phi_{0}\right)}{6 \sqrt{2}}\left(b_{1}^{1}+b_{1}^{-1}\right)+\frac{4 \eta_{2} c_{1}^{1} \sin \phi_{0} \mathbf{i}}{6 \sqrt{2}}\left(b_{1}^{1}-b_{1}^{-1}\right) .
\end{aligned}
$$

Assume that there exists $n \in \mathbb{N} \backslash\{1\}$ such that

$$
a_{l}^{0}=b_{l}^{0}=a_{l}^{ \pm 1}=b_{l}^{ \pm 1}=0, \quad l=1, \ldots, n-1 .
$$

Then we have

$$
\begin{aligned}
& \frac{\eta_{1} \sqrt{n(n+1)} c_{n}^{0}}{2 n+1} b_{n}^{0}=\frac{\mathbf{i} k n(n+1)^{2} c_{n}^{1} \sin ^{2} \phi_{0}}{2(2 n+1) \sqrt{n(n+1)}}\left(a_{n}^{1}+a_{n}^{-1}\right) \\
& -\frac{k n(n+1)^{2} c_{n}^{1} \sin \phi_{0} \cos \phi_{0}}{2(2 n+1) \sqrt{n(n+1)}}\left(a_{n}^{1}-a_{n}^{-1}\right)-\frac{\eta_{2} \sqrt{n(n+1)} c_{n}^{0} \cos \phi_{0}}{2 n+1} b_{n}^{0}
\end{aligned}
$$




$$
\begin{aligned}
& \frac{k n(n+1)^{2} c_{n}^{1}}{2(2 n+1) \sqrt{n(n+1)}}\left(a_{n}^{1}-a_{n}^{-1}\right)=-\frac{\mathbf{i} k n(n+1)^{2} c_{n}^{1} \sin \phi_{0} \cos \phi_{0}}{2(2 n+1) \sqrt{n(n+1)}}\left(a_{n}^{1}+a_{n}^{-1}\right) \\
& \quad+\frac{k n(n+1)^{2} c_{n}^{1} \cos ^{2} \phi_{0}}{2(2 n+1) \sqrt{n(n+1)}}\left(a_{n}^{1}-a_{n}^{-1}\right)-\frac{\eta_{2} \sqrt{n(n+1)} c_{n}^{0} \sin \phi_{0}}{2 n+1} b_{n}^{0} \\
& -\frac{\eta_{1} n(n+1)^{2} c_{n}^{1}}{2(2 n+1) \sqrt{n(n+1)}}\left(b_{n}^{1}+b_{n}^{-1}\right)=\frac{\eta_{2} n(n+1)^{2} c_{n}^{1} \cos \phi_{0}}{2(2 n+1) \sqrt{n(n+1)}}\left(b_{n}^{1}+b_{n}^{-1}\right) \\
& \quad-\frac{n(n+1)^{2} \eta_{2} \sin \phi_{0} \mathbf{i}}{2(2 n+1) \sqrt{n(n+1)}}\left(b_{n}^{1}-b_{n}^{-1}\right) .
\end{aligned}
$$

Proof. We shall first derive (3.4a), (3.4b) and (3.4c). (3.2a), (3.2b) and (3.2c) can be obtained in a similar way and we shall sketch the corresponding derivations at the end of the proof.

We first note that

$$
\left(\nu_{2} \wedge \mathbf{E}\right) \wedge \nu_{2}=-\nu_{2} \wedge\left(\nu_{2} \wedge \mathbf{E}\right)=-\left(\nu_{2} \cdot\left(\nu_{2} \cdot \mathbf{E}\right)-\mathbf{E}\left(\nu_{2} \cdot \nu_{2}\right)\right)=\mathbf{E}-\left(\nu_{2} \cdot \mathbf{E}\right) \cdot \nu_{2}
$$

Hence, we have from

$$
\nu_{2} \wedge\left(\left.\nabla \wedge \mathbf{E}\right|_{\widetilde{\Pi}_{2}}\right)+\boldsymbol{\eta}_{2}\left(\left.\nu_{2} \wedge \mathbf{E}\right|_{\widetilde{\Pi}_{2}}\right) \wedge \nu_{2}=\mathbf{0}
$$

that

$$
\left.\nu_{2} \wedge(\nabla \wedge \mathbf{E})\right|_{\widetilde{\Pi}_{2}}+\boldsymbol{\eta}_{2}\left(\left.\mathbf{E}\right|_{\widetilde{\Pi}_{2}}-\left(\left.\nu_{2} \cdot \mathbf{E}\right|_{\widetilde{\Pi}_{2}}\right) \cdot \nu_{2}\right)=\mathbf{0} .
$$

Multiplying the cross product with $\nu_{2}$ from left on both sides (3.7), by using the fact that

$$
\nu_{2} \wedge\left(\left.\nu_{2} \wedge(\nabla \wedge \mathbf{E})\right|_{\widetilde{\Pi}_{2}}\right)=\nu_{2} \cdot\left(\left.\nu_{2} \cdot(\nabla \wedge \mathbf{E})\right|_{\widetilde{\Pi}_{2}}\right)-\left.\left(\nu_{2} \cdot \nu_{2}\right)(\nabla \wedge \mathbf{E})\right|_{\widetilde{\Pi}_{2}},
$$

we can obtain that

$$
\left(\left.\nu_{2} \cdot(\nabla \wedge \mathbf{E})\right|_{\widetilde{\Pi}_{2}}\right) \nu_{2}+\boldsymbol{\eta}_{2}\left(\left.\nu_{2} \wedge \mathbf{E}\right|_{\widetilde{\Pi}_{2}}\right)=\left.\nabla \wedge \mathbf{E}\right|_{\widetilde{\Pi}_{2}}
$$

Similarly, since the generalized impedance condition 1.3 associated with $\boldsymbol{\eta}_{1}$ is imposed on $\widetilde{\Pi}_{1}$, using the above argument, we can deduce that

$$
\left(\left.\nu_{1} \cdot(\nabla \wedge \mathbf{E})\right|_{\widetilde{\Pi}_{1}}\right) \nu_{1}+\boldsymbol{\eta}_{1}\left(\left.\nu_{1} \wedge \mathbf{E}\right|_{\widetilde{\Pi}_{1}}\right)=\left.\nabla \wedge \mathbf{E}\right|_{\widetilde{\Pi}_{1}} .
$$

Since $\boldsymbol{l} \in \widetilde{\Pi}_{1} \cap \widetilde{\Pi}_{2}$, combing 3.8$)$ with 3.9$)$, it yields that

$$
\left(\nu_{1} \cdot\left(\left.\nabla \wedge \mathbf{E}\right|_{l}\right)\right) \nu_{1}+\boldsymbol{\eta}_{1}\left(\left.\nu_{1} \wedge \mathbf{E}\right|_{l}\right)=\left(\nu_{2} \cdot\left(\left.\nabla \wedge \mathbf{E}\right|_{l}\right)\right) \nu_{2}+\boldsymbol{\eta}_{2}\left(\left.\nu_{2} \wedge \mathbf{E}\right|_{l}\right) .
$$

Due to (3.3), using (2.24) and (2.28), by virtue of (2.10), it yields that

$$
\begin{aligned}
\left.\nabla \wedge \mathbf{E}\right|_{\widetilde{\Pi}_{2}}= & \sum_{l=n}^{\infty} \sum_{m=-l}^{l} \frac{\mathbf{i} k}{\sqrt{l(l+1)}}\left\{\left.a_{l}^{m} \cdot l(l+1) p_{l}(k r) c_{l}^{m} P_{l}^{|m|} \cdot \hat{\boldsymbol{r}}\right|_{\widetilde{\Pi}_{2}}\right. \\
& +\left(b_{l}^{m} j_{l}(k r) c_{l}^{m} \frac{\operatorname{sgn}(m)}{2}\left[P_{l-1}^{|m|+1}(\cos \theta)+(l+|m|-1)(l+|m|) P_{l-1}^{|m|-1}(\cos \theta)\right]\right. \\
& \left.+a_{l}^{m} q_{l}(k r) c_{l}^{m} \frac{1}{2}\left[(l+|m|)(l-|m|+1) P_{l}^{|m|-1}(\cos \theta)-P_{l}^{|m|+1}(\cos \theta)\right]\right)\left.\hat{\boldsymbol{\theta}}\right|_{\widetilde{\Pi}_{2}} \\
& +\left(-b_{l}^{m} j_{l}(k r) \mathbf{i} c_{l}^{m} \frac{1}{2}\left[(l+|m|)(l-|m|+1) P_{l}^{|m|-1}(\cos \theta)-P_{l}^{|m|+1}(\cos \theta)\right]\right. \\
& -a_{l}^{m} \cdot q_{l}(k r) \mathbf{i} c_{l}^{m} \frac{\operatorname{sgn}(m)}{2}\left[P_{l-1}^{|m|+1}(\cos \theta)\right. \\
& \left.\left.\left.+(l+|m|-1)(l+|m|) P_{l-1}^{|m|-1}(\cos \theta)\right]\right)\left.\hat{\boldsymbol{\phi}}\right|_{\Pi_{2}}\right\}
\end{aligned}
$$


and

$$
\begin{aligned}
\left.\nu_{2} \wedge \mathbf{E}\right|_{\widetilde{\Pi}_{2}} & =\sum_{l=n}^{\infty} \sum_{m=-l}^{l}\left\{\left\{-b_{l}^{m} \cdot \sqrt{l(l+1)} p_{l}(k r) \cdot c_{l}^{m} P_{l}^{|m|}\right\} \cdot \nu_{2} \wedge \hat{\boldsymbol{r}}_{\widetilde{\Pi}_{2}}\right. \\
& +\left\{a _ { l } ^ { m } \cdot j _ { l } ( k r ) \frac { 1 } { \sqrt { l ( l + 1 ) } } c _ { l } ^ { m } \frac { \operatorname { s g n } ( m ) } { 2 } \left[P_{l-1}^{|m|+1}(\cos \theta)+(l+|m|-1)(l+|m|)\right.\right. \\
& \left.\times P_{l-1}^{|m|-1}(\cos \theta)\right]-b_{l}^{m} \cdot q_{l}(k r) \cdot \frac{1}{\sqrt{l(l+1)}} c_{l}^{m} \frac{1}{2}[(l+|m|)(l-|m|+1) \\
& \left.\left.\times P_{l}^{|m|-1}(\cos \theta)-P_{l}^{|m|+1}(\cos \theta)\right]\right\}\left.\cdot \nu_{2} \wedge \hat{\boldsymbol{\theta}}\right|_{\widetilde{\Pi}_{2}}+\left\{-a_{l}^{m} \cdot j_{l}(k r) \frac{\mathbf{i}}{\sqrt{l(l+1)}} \frac{c_{l}^{m}}{2}\right. \\
& \times\left[(l+|m|)(l-|m|+1) P_{l}^{|m|-1}(\cos \theta)-P_{l}^{|m|+1}(\cos \theta)\right]+b_{l}^{m} q_{l}(k r) \frac{\mathbf{i}}{\sqrt{l(l+1)}} \\
& \left.\left.\times c_{l}^{m} \frac{\operatorname{sgn}(m)}{2}\left[P_{l-1}^{|m|+1}(\cos \theta)+(l+|m|-1)(l+|m|) P_{l-1}^{|m|-1}(\cos \theta)\right]\right\} \times\left.\nu_{2} \wedge \hat{\boldsymbol{\phi}}\right|_{\widetilde{\Pi}_{2}}\right\},
\end{aligned}
$$

where

$$
\operatorname{sgn}(m)=1 \text { when } m>0 ; 0 \text { when } m=0 ;-1 \text { when } m<0 .
$$

Recall that if $\mathrm{x} \in \boldsymbol{l}$ one has

$$
\theta=\phi=0, \quad 0 \leq r \leq h,
$$

where $r, \theta$ and $\phi$ are the spherical coordinates of $\mathbf{x} \in \boldsymbol{l}$ defined in 2.4. It is straightforward to calculate that

$$
\begin{aligned}
& \left.\nu_{2} \wedge \hat{\boldsymbol{r}}\right|_{\theta=\phi=0}=\left[\begin{array}{c}
\cos \phi_{0} \\
\sin \phi_{0} \\
0
\end{array}\right],\left.\nu_{2} \wedge \hat{\boldsymbol{\theta}}\right|_{\theta=\phi=0}=-\left[\begin{array}{c}
0 \\
0 \\
\cos \phi_{0}
\end{array}\right],\left.\nu_{2} \wedge \hat{\boldsymbol{\phi}}\right|_{\theta=\phi=0}=-\left[\begin{array}{c}
0 \\
0 \\
\sin \phi_{0}
\end{array}\right], \\
& \left.\nu_{2} \cdot \hat{\boldsymbol{r}}\right|_{\theta=\phi=0}=0,\left.\quad \nu_{2} \cdot \hat{\boldsymbol{\theta}}\right|_{\theta=\phi=0}=-\sin \phi_{0},\left.\quad \nu_{2} \cdot \hat{\boldsymbol{\phi}}\right|_{\theta=\phi=0}=\cos \phi_{0},
\end{aligned}
$$

where $\hat{\boldsymbol{r}}, \hat{\boldsymbol{\theta}}$ and $\hat{\boldsymbol{\phi}}$ are defined in $(2.4)$.

Evaluating (3.11) and (3.12) at $\boldsymbol{l}$, by virtue of (2.11) and (3.14), we can derive that

$$
\begin{aligned}
\left.\nabla \wedge \mathbf{E}\right|_{l} & =\sum_{l=n}^{+\infty} \frac{\mathbf{i} k}{\sqrt{l(l+1)}}\left\{\left.a_{l}^{0} l(l+1) p_{l}(k r) c_{l}^{0} \cdot \hat{\boldsymbol{r}}\right|_{\theta=\phi=0}\right. \\
& +\left.\frac{(l+1) l}{2}\left(\left(b_{l}^{1}-b_{l}^{-1}\right) \cdot j_{l}(k r) \cdot c_{l}^{1}+\left(a_{l}^{1}+a_{l}^{-1}\right) q_{l}(k r) c_{l}^{1}\right) \cdot \hat{\boldsymbol{\theta}}\right|_{\theta=\phi=0} \\
& \left.+\left.\mathbf{i} \frac{(l+1) l}{2}\left(-\left(b_{l}^{1}+b_{l}^{-1}\right) \cdot j_{l}(k r) c_{l}^{1}-\left(a_{l}^{1}-a_{l}^{-1}\right) q_{l}(k r) c_{l}^{1}\right) \cdot \hat{\boldsymbol{\phi}}\right|_{\theta=\phi=0}\right\} \\
\left.\nu_{2} \wedge \mathbf{E}\right|_{l} & =\sum_{l=n}^{+\infty} \frac{1}{\sqrt{l(l+1)}}\left\{\left(-\left.b_{l}^{0} l(l+1) p_{l}(k r) c_{l}^{0} \cdot \nu_{2} \wedge \hat{\boldsymbol{r}}\right|_{\theta=\phi=0}\right.\right. \\
& -\left.\frac{(l+1) l}{2}\left(-\left(a_{l}^{1}-a_{l}^{-1}\right) j_{l}(k r) c_{l}^{1}+\left(b_{l}^{1}+b_{l}^{-1}\right) q_{l}(k r) c_{l}^{1}\right) \cdot \nu_{2} \wedge \hat{\boldsymbol{\theta}}\right|_{\theta=\phi=0} \\
& \left.\left.-\left.\mathbf{i} \frac{(l+1) l}{2}\left(\left(a_{l}^{1}+a_{l}^{-1}\right) j_{l}(k r) c_{l}^{1}-\left(b_{l}^{1}-b_{l}^{-1}\right) q_{l}(k r) c_{l}^{1}\right) \cdot \nu_{2} \cdot \hat{\boldsymbol{\phi}}\right|_{\theta=\phi=0}\right)\right\} .
\end{aligned}
$$


Therefore, from (3.15) we obtain that

$$
\begin{aligned}
& \nu_{2}^{\top}\left(\left.\nabla \wedge \mathbf{E}\right|_{\boldsymbol{l}}\right) \nu_{2}+\boldsymbol{\eta}_{2}\left(\left.\nu_{2} \wedge \mathbf{E}\right|_{\boldsymbol{l}}\right) \\
& =\sum_{l=n}^{+\infty} \frac{1}{\sqrt{l(l+1)}}\left\{\mathbf { i } k \left[-\sin \phi_{0} \frac{c_{l}^{1}(l+1) l}{2}\left(\left(b_{l}^{1}-b_{l}^{-1}\right) j_{l}(k r)+\left(a_{l}^{1}+a_{l}^{-1}\right) q_{l}(k r) c_{l}^{1}\right)\right.\right. \\
& \left.-\cos \phi_{0} \frac{\mathbf{i}(l+1) l}{2} \times\left(\left(b_{l}^{1}+b_{l}^{-1}\right) j_{l}(k r) c_{l}^{1}+\left(a_{l}^{1}-a_{l}^{-1}\right) q_{l}(k r) c_{l}^{1}\right)\right] \\
& \times\left[\begin{array}{c}
-\sin \phi_{0} \\
\cos \phi_{0} \\
0
\end{array}\right]+\boldsymbol{\eta}_{2}\left[-b_{l}^{0} l(l+1) p_{l}(k r) c_{l}^{0}\left[\begin{array}{c}
\cos \phi_{0} \\
\sin \phi_{0} \\
0
\end{array}\right]\right. \\
& -\frac{(l+1) l}{2}\left(-\left(a_{l}^{1}-a_{l}^{-1}\right) j_{l}(k r) c_{l}^{1}+\left(b_{l}^{1}+b_{l}^{-1}\right) q_{l}(k r) c_{l}^{1}\right)\left[\begin{array}{c}
0 \\
0 \\
-\cos \phi_{0}
\end{array}\right] \\
& \left.\left.-\frac{\mathbf{i}(l+1) l}{2}\left(\left(a_{l}^{1}+a_{l}^{-1}\right) j_{l}(k r) c_{l}^{1}-\left(b_{l}^{1}-b_{l}^{-1}\right) q_{l}(k r) c_{l}^{1}\right) \cdot\left[\begin{array}{c}
0 \\
0 \\
-\sin \phi_{0}
\end{array}\right]\right]\right\} \text {. } \\
& \nu_{2}^{\top}\left(\left.\nabla \wedge \mathbf{E}\right|_{\boldsymbol{l}}\right) \nu_{2}+\boldsymbol{\eta}_{2}\left(\left.\nu_{2} \wedge \mathbf{E}\right|_{\boldsymbol{l}}\right) \\
& =\sum_{l=n}^{+\infty} \frac{1}{\sqrt{l(l+1)}}\left\{\mathbf { i } k \left[-\sin \phi_{0} \frac{c_{l}^{1}(l+1) l}{2}\left(\left(b_{l}^{1}-b_{l}^{-1}\right) j_{l}(k r)+\left(a_{l}^{1}+a_{l}^{-1}\right) q_{l}(k r) c_{l}^{1}\right)\right.\right. \\
& \left.-\cos \phi_{0} \frac{\mathbf{i}(l+1) l}{2} \times\left(\left(b_{l}^{1}+b_{l}^{-1}\right) j_{l}(k r) c_{l}^{1}+\left(a_{l}^{1}-a_{l}^{-1}\right) q_{l}(k r) c_{l}^{1}\right)\right] \\
& \times\left[\begin{array}{c}
-\sin \phi_{0} \\
\cos \phi_{0} \\
0
\end{array}\right]+\boldsymbol{\eta}_{2}\left[-b_{l}^{0} l(l+1) p_{l}(k r) c_{l}^{0}\left[\begin{array}{c}
\cos \phi_{0} \\
\sin \phi_{0} \\
0
\end{array}\right]\right. \\
& -\frac{(l+1) l}{2}\left(-\left(a_{l}^{1}-a_{l}^{-1}\right) j_{l}(k r) c_{l}^{1}+\left(b_{l}^{1}+b_{l}^{-1}\right) q_{l}(k r) c_{l}^{1}\right)\left[\begin{array}{c}
0 \\
0 \\
-\cos \phi_{0}
\end{array}\right] \\
& \left.\left.-\frac{\mathbf{i}(l+1) l}{2}\left(\left(a_{l}^{1}+a_{l}^{-1}\right) j_{l}(k r) c_{l}^{1}-\left(b_{l}^{1}-b_{l}^{-1}\right) q_{l}(k r) c_{l}^{1}\right) \cdot\left[\begin{array}{c}
0 \\
0 \\
-\sin \phi_{0}
\end{array}\right]\right]\right\} .
\end{aligned}
$$

Using a similar argument for deriving (3.17), we have

$$
\begin{aligned}
& \nu_{1}^{\top}\left(\left.\nabla \wedge \mathbf{E}\right|_{l}\right) \nu_{1}+\eta_{1}\left(\left.\nu \wedge \mathbf{E}\right|_{l}\right) \\
= & -\sum_{l=n}^{+\infty}\left\{\frac{k l(l+1)}{2 \sqrt{l(l+1)}}\left[\left(b_{l}^{1}+b_{l}^{-1}\right) \cdot j_{l}(k r) \cdot c_{l}^{1}+\left(a_{l}^{1}-a_{l}^{-1}\right) q_{l}(k r) c_{l}^{1}\right]\left[\begin{array}{c}
0 \\
-1 \\
0
\end{array}\right]\right. \\
& +\boldsymbol{\eta}_{1}\left(-b_{l}^{0} \sqrt{l(l+1)} p_{l}(k r) c_{l}^{0}\left[\begin{array}{c}
-1 \\
0 \\
0
\end{array}\right]+\frac{l(l+1)}{2 \sqrt{l(l+1)}}\left(\left(a_{l}^{1}-a_{l}^{-1}\right) j_{l}(k r) c_{l}^{1}\right.\right. \\
& \left.\left.\left.-\left(b_{l}^{1}+b_{l}^{-1}\right) q_{l}(k r) c_{l}^{1}\right)\left[\begin{array}{l}
0 \\
0 \\
1
\end{array}\right]\right)\right\} .
\end{aligned}
$$

Note that $\boldsymbol{\eta}_{\ell}, \ell=1,2$, have the expansions (3.1a) and (3.1b respectively, where the coefficients of $r^{0}$ in (3.1a) and (3.1b) are the non-zero numbers $\eta_{1}$ and $\eta_{2}$. From Remark 
2.6, it is easy to see that the lowest order of (3.17) and (3.18) with respect to the power of $r$ is $n-1$, which is contributed by $p_{n}(k r)$ and $q_{n}(k r)$ in (3.17) and (3.18). Substituting (3.17) and 3.18 into 3.10), and comparing the coefficients of $r^{n-1}$ on both sides of the first, second and third component of 3.10 respectively, we can derive $3.4 \mathrm{a}),(3.4 \mathrm{~b})$ and (3.4c).

We can derive $(3.2 \mathrm{a}),(3.2 \mathrm{~b})$ and $(3.2 \mathrm{c})$ by similar arguments for $3.4 \mathrm{a}),(3.4 \mathrm{~b})$ and (3.4c). Indeed, the Fourier expansions of (3.17) and (3.18) can be rewritten with the starting summation index $n=1$. Hence we can obtain (3.2a), (3.2b) and (3.2c) by comparing the coefficients of $r^{0}$ on both sides of (3.10) by virtue of (3.17) and (3.18) .

The proof is complete.

Lemma 3.2. Under the same setup in Lemma 3.1, it holds that

$$
\begin{aligned}
& 0=-\frac{4 \eta_{2} c_{1}^{1} \cos ^{2} \phi_{0}}{6 \sqrt{2}}\left(b_{1}^{1}+b_{1}^{-1}\right)+\frac{4 \mathbf{i} \eta_{2} c_{1}^{1} \sin \phi_{0} \cos \phi_{0}}{6 \sqrt{2}}\left(b_{1}^{1}-b_{1}^{-1}\right)+\frac{\mathbf{i} k \sqrt{2} c_{1}^{0} \cos \phi_{0}}{3} a_{1}^{0}, \\
& 0=\frac{4 \eta_{2} c_{1}^{1} \sin \phi_{0} \cos \phi_{0}}{6 \sqrt{2}}\left(b_{1}^{1}+b_{1}^{-1}\right)+\frac{4 \mathbf{i} \eta_{2} c_{1}^{1} \sin ^{2} \phi_{0}}{6 \sqrt{2}}\left(b_{1}^{1}-b_{1}^{-1}\right)+\frac{\mathbf{i} k \sqrt{2} c_{1}^{0} \sin \phi_{0}}{3} a_{1}^{0}, \\
& 0=\frac{4 \mathbf{i} k c_{1}^{1} \cos \phi_{0}}{6 \sqrt{2}}\left(a_{1}^{1}+a_{1}^{-1}\right)+\frac{4 k c_{1}^{1} \sin \phi_{0}}{6 \sqrt{2}}\left(a_{1}^{1}-a_{1}^{-1}\right)+\frac{\eta_{2} \sqrt{2} c_{1}^{0}}{3} b_{1}^{0} .
\end{aligned}
$$

Furthermore, if we assume that there exists $n \in \mathbb{N} \backslash\{1\}$ such that (3.3) is fulfilled, then it holds that

$$
\begin{aligned}
0= & \frac{\mathbf{i} k \sqrt{n(n+1)} c_{n}^{0} \cos \phi_{0}}{2 n+1} a_{n}^{0}-\frac{\eta_{2} n(n+1)^{2} c_{n}^{1} \cos ^{2} \phi_{0}}{2(2 n+1) \sqrt{n(n+1)}}\left(b_{n}^{1}+b_{n}^{-1}\right) \\
& +\frac{\mathbf{i} \eta_{2} n(n+1)^{2} c_{n}^{1} \sin \phi_{0} \cos \phi_{0}}{2(2 n+1) \sqrt{n(n+1)}}\left(b_{n}^{1}-b_{n}^{-1}\right), \\
0= & \frac{\mathbf{i} k c_{n}^{0} \sqrt{n(n+1)} \sin \phi_{0}}{2 n+1} a_{n}^{0}+\frac{\eta_{2} n(n+1)^{2} c_{n}^{1} \sin \phi_{0} \cos \phi_{0}}{2(2 n+1) \sqrt{n(n+1)}}\left(b_{n}^{1}+b_{n}^{-1}\right) \\
& +\frac{\mathbf{i} \eta_{2} n(n+1)^{2} c_{n}^{1} \sin ^{2} \phi_{0}}{2(2 n+1) \sqrt{n(n+1)}}\left(b_{n}^{1}-b_{n}^{-1}\right), \\
0= & \frac{\mathbf{i} k n(n+1)^{2} c_{n}^{1} \cos \phi_{0}}{2(2 n+1) \sqrt{n(n+1)}}\left(a_{n}^{1}+a_{n}^{-1}\right)+\frac{k n(n+1)^{2} c_{n}^{1} \sin \phi_{0}}{2(2 n+1) \sqrt{n(n+1)}}\left(a_{n}^{1}-a_{n}^{-1}\right) \\
& +\frac{\eta_{2} \sqrt{n(n+1)} c_{n}^{0}}{2 n+1} b_{n}^{0} .
\end{aligned}
$$

Proof. We first prove $3.21 \mathrm{a}, 3.21 \mathrm{~b}$ ) and $3.21 \mathrm{c}$. Since the generalized impedance condition 1.3 associated with $\boldsymbol{\eta}_{2}$ is imposed on $\widetilde{\Pi}_{2}$, we have

$$
\nu_{2} \wedge\left(\left.\nabla \wedge \mathbf{E}\right|_{l}\right)+\boldsymbol{\eta}_{2}\left(\left.\nu_{2} \wedge \mathbf{E}\right|_{l}\right) \wedge \nu_{2}=\mathbf{0} .
$$

Here, we recall (3.13). Under the assumption (3.3), using (2.10), 2.11) and (2.31), we can obtain that

$$
\begin{aligned}
& \nu_{2} \wedge\left(\left.\nabla \wedge \mathbf{E}\right|_{l}\right)+\eta_{2}\left(\left.\nu_{2} \wedge \mathbf{E}\right|_{l}\right) \wedge \nu_{2}=\sum_{l=n}^{+\infty}\left\{\mathbf { i } k \left\{a_{l}^{0} \sqrt{l(l+1)} p_{l}(k r) c_{l}^{0} \cdot\left(\cos \phi_{0}, \sin \phi_{0}, 0\right)^{\top}\right.\right. \\
& +\frac{l(l+1)}{2 \sqrt{l(l+1)}}\left(\left(b_{l}^{1}-b_{l}^{-1}\right) j_{l}(k r) c_{l}^{1}+\left(a_{l}^{1}+a_{l}^{-1}\right) q_{l}(k r) c_{l}^{1}\right)\left[\begin{array}{c}
0 \\
0 \\
-\cos \phi_{0}
\end{array}\right]-\frac{l(l+1) \mathbf{i}}{2 \sqrt{l(l+1)}}\left(\left(b_{l}^{1}+b_{l}^{-1}\right)\right.
\end{aligned}
$$




$$
\begin{aligned}
& \left.\left.\times j_{l}(k r) c_{l}^{1}+\left(a_{l}^{1}-a_{l}^{-1}\right) q_{l}(k r) c_{l}^{1}\right)\left[\begin{array}{c}
0 \\
0 \\
-\sin \phi_{0}
\end{array}\right]\right\}+\boldsymbol{\eta}_{2}\left\{-b_{l}^{0} \sqrt{l(l+1)} p_{l}(k r) c_{l}^{0}\left[\begin{array}{l}
0 \\
0 \\
1
\end{array}\right]+\frac{l(l+1)}{2 \sqrt{l(l+1)}}\right. \\
& \times\left(\left(a_{l}^{1}-a_{l}^{-1}\right) j_{l}(k r) c_{l}^{1}+\left(b_{l}^{1}+b_{l}^{-1}\right) q_{l}(k r) c_{l}^{1}\right) \times\left[\begin{array}{c}
\cos ^{2} \phi_{0} \\
-\sin \phi_{0} \cos \phi_{0} \\
0
\end{array}\right]+\frac{l(l+1) \mathbf{i}}{2 \sqrt{l(l+1)}}\left(\left(a_{l}^{1}+a_{l}^{-1}\right)\right. \\
& \left.\left.\times j_{l}(k r) c_{l}^{1}-\left(b_{l}^{1}-b_{l}^{-1}\right) q_{l}(k r) c_{l}^{1}\right) \times\left[\begin{array}{c}
\sin \phi_{0} \cos \phi_{0} \\
\sin ^{2} \phi_{0} \\
0
\end{array}\right]\right\} .
\end{aligned}
$$

Note that $\boldsymbol{\eta}_{\ell}, \ell=1,2$, have the expansions (3.1a) and (3.1b) respectively, where the coefficients of $r^{0}$ in (3.1a) and (3.1b) are non zero number $\eta_{1}$ and $\eta_{2}$. In view of Remark 2.6 . we know that the lowest order of (3.23) with respect to the power of $r$ is $n-1$, which is contributed by $p_{n}(k r)$ and $q_{n}(k r)$ in 3.23$)$. Substituting (3.17) and (3.23) into (3.22), comparing the coefficients of $r^{n-1}$ on both sides of the first, the second, the third, component of (3.22) respectively, we derive that (3.21a), (3.21b) and (3.21c).

We can derive (3.18), (3.19) and (3.20) by following similar arguments in deriving (3.21a), (3.21b) and (3.21c). Indeed, the Fourier expansions of $(3.23$ can be rewritten with the starting summation index $n=1$. Hence we can obtain (3.18), (3.19) and (3.20) by comparing the coefficients of $r^{0}$ on both sides of 3.22 by virtue of (3.23).

Lemma 3.3. Under the same setup in Lemma 3.1, one has the following linear relations:

$$
\left\{\begin{array}{l}
\beta_{11}^{1}\left(b_{1}^{1}+b_{1}^{-1}\right)+\beta_{12}^{1}\left(b_{1}^{1}-b_{1}^{-1}\right)+\beta_{13}^{1} a_{1}^{0}=0 \\
\beta_{21}^{1}\left(b_{1}^{1}+b_{1}^{-1}\right)+\beta_{22}^{1}\left(b_{1}^{1}-b_{1}^{-1}\right)+\beta_{23}^{1} a_{1}^{0}=0 \\
\beta_{31}^{1}\left(b_{1}^{1}+b_{1}^{-1}\right)+\beta_{32}^{1}\left(b_{1}^{1}-b_{1}^{-1}\right)+\beta_{33}^{1} a_{1}^{0}=0
\end{array}\right.
$$

where

$$
\begin{aligned}
& \beta_{11}^{1}=-\frac{4 \eta_{2} c_{1}^{1} \cos ^{2} \phi_{0}}{6 \sqrt{2}}, \quad \beta_{12}^{1}=\frac{4 \mathbf{i} \eta_{2} c_{1}^{1} \sin \phi_{0} \cos \phi_{0}}{6 \sqrt{2}}, \quad \beta_{13}^{1}=\frac{\mathbf{i} k \sqrt{2} c_{1}^{0} \cos \phi_{0}}{3}, \\
& \beta_{21}^{1}=\frac{4 \eta_{2} c_{1}^{1} \sin \phi_{0} \cos \phi_{0}}{6 \sqrt{2}}, \quad \beta_{22}^{1}=\frac{4 \mathbf{i} \eta_{2} c_{1}^{1} \sin ^{2} \phi_{0}}{6 \sqrt{2}}, \quad \beta_{23}^{1}=\frac{\mathbf{i} k \sqrt{2} c_{1}^{0} \sin \phi_{0}}{3}, \\
& \beta_{31}^{1}=-\frac{4 c_{1}^{1}\left(-\eta_{1}+\eta_{2} \cos \phi_{0}\right)}{6 \sqrt{2}}, \quad \beta_{32}^{1}=\frac{4 \eta_{2} c_{1}^{1} \sin \phi_{0} \mathbf{i}}{6 \sqrt{2}}, \quad \beta_{33}^{1}=0 .
\end{aligned}
$$

If we assume that there exists $n \in \mathbb{N} \backslash\{1\}$ such that (3.3) is fulfilled, then one has that

$$
\left\{\begin{array}{l}
\beta_{11}^{n}\left(b_{n}^{1}+b_{n}^{-1}\right)+\beta_{12}^{n}\left(b_{n}^{1}-b_{n}^{-1}\right)+\beta_{13}^{n} a_{n}^{0}=0, \\
\beta_{21}^{n}\left(b_{n}^{1}+b_{n}^{-1}\right)+\beta_{22}^{n}\left(b_{n}^{1}-b_{n}^{-1}\right)+\beta_{23}^{n} a_{n}^{0}=0, \\
\beta_{31}^{n}\left(b_{n}^{1}+b_{n}^{-1}\right)+\beta_{32}^{n}\left(b_{n}^{1}-b_{n}^{-1}\right)+\beta_{33}^{n} a_{n}^{0}=0,
\end{array}\right.
$$

where

$$
\begin{aligned}
& \beta_{11}^{n}=-\frac{\eta_{2} n(n+1)^{2} c_{n}^{1} \cos ^{2} \phi_{0}}{2(2 n+1) \sqrt{n(n+1)}}, \\
& \beta_{12}^{n}=\frac{\mathbf{i} \eta_{2} n(n+1)^{2} c_{n}^{1} \sin \phi_{0} \cos \phi_{0}}{2(2 n+1) \sqrt{n(n+1)}}, \quad \beta_{13}^{n}=\frac{\mathbf{i} k \sqrt{n(n+1)} c_{n}^{0} \cos \phi_{0}}{2 n+1}, \\
& \beta_{21}^{n}=\frac{\eta_{2} n(n+1)^{2} c_{n}^{1} \sin \phi_{0} \cos \phi_{0}}{2(2 n+1) \sqrt{n(n+1)}}, \quad \beta_{22}^{n}=\frac{\mathbf{i} \eta_{2} n(n+1)^{2} c_{n}^{1} \sin ^{2} \phi_{0}}{2(2 n+1) \sqrt{n(n+1)}}, \\
& \beta_{23}^{n}=\frac{\mathbf{i} k \sqrt{n(n+1)} c_{n}^{0} \sin \phi_{0}}{2 n+1}, \quad \beta_{31}^{n}=-\frac{n(n+1)^{2} c_{n}^{1}\left(-\eta_{1}+\eta_{2} \cos \phi_{0}\right)}{2(2 n+1) \sqrt{n(n+1)}},
\end{aligned}
$$




$$
\beta_{32}^{n}=\frac{\mathbf{i} \eta_{2} n(n+1)^{2} c_{n}^{1} \sin \phi_{0}}{2(2 n+1) \sqrt{n(n+1)}}, \quad \beta_{33}^{n}=0 .
$$

Furthermore, if $\alpha \neq \frac{1}{2}$ and $\alpha \neq \frac{3}{2}$, then it holds that

$$
a_{n}^{0}=b_{n}^{ \pm 1}=0 .
$$

Proof. Combining (3.18), (3.19) with (3.2c), we can obtain (3.24). Similarly, by virtue of (3.4c), (3.21a) and (3.21b), we can derive (3.26). After straightforward calculations, it can be verified that the determinant of coefficients matrices $(3.26)$ is given by

$$
\left|\mathcal{B}_{n}\right|=-k \eta_{2}^{2}\left(\frac{n+1}{2 n+1}\right)^{3} \frac{n \sqrt{n(n+1)}}{2}\left(c_{n}^{1}\right)^{2} c_{n}^{0} \sin ^{2} \phi_{0} \cos ^{2} \phi_{0}
$$

where $c_{n}^{0}, c_{n}^{1}$ are nonzero constants defined in (2.8). Recall that $\boldsymbol{\eta}_{\ell}, \ell=1,2$, have the expansions (3.1a) and (3.1b) respectively, where the coefficients of $r^{0}$ in $3.1 \mathrm{a}$ ) and $(3.1 \mathrm{~b})$ are non zero number $\eta_{1}$ and $\eta_{2}$. Since $\phi_{0}=\alpha \pi \neq \pi / 2, \phi_{0}=\alpha \pi \neq 3 \pi / 2, \eta_{2} \neq 0$ and $k \in \mathbb{R}_{+}$, we conclude that $\mathcal{B}_{n}$ are nonsingular, which readily implies (3.27).

The following two important lemmas reveal the recursive relationships for $a_{n}^{ \pm m}$ and $b_{n}^{ \pm m}$, where $m=0,1, \ldots, n$, which will be used to characterize the vanishing order of $\mathbf{E}$ with respect to the the corresponding dihedral angle of the edge-corner $\mathcal{E}\left(\widetilde{\Pi}_{1}, \widetilde{\Pi}_{2}, \boldsymbol{l}\right) \Subset \Omega$ in Theorem 3.6 .

Lemma 3.4. Let $\mathbf{E}$ be a a solution to (1.1), whose radial wave expansion in $B_{\rho_{0}}(\mathbf{0})$ is given by 2.17). Consider a generalized impedance edge-corner $\mathcal{E}\left(\widetilde{\Pi}_{1}, \widetilde{\Pi}_{2}, \boldsymbol{l}\right) \Subset \Omega$ with $\angle\left(\Pi_{1}, \Pi_{2}\right)=\phi_{0}=\alpha \pi$, where $\alpha \in(0,2)$ and $\alpha \neq 1$. Suppose that the generalized impedance parameters $\boldsymbol{\eta}_{j}$ on $\widetilde{\Pi}_{j}, j=1,2$, are given by 3.1a) and $3.1 \mathrm{~b}$ respectively. Assume that there exists $n \in \mathbb{N} \backslash\{1\}$ such that

$$
a_{l}^{m}=b_{l}^{m}=0, \quad l=1, \ldots, n-1, \text { and } m \in[l]_{0} .
$$

Then we have the following recursive linear equations:

$$
\left\{\begin{aligned}
0= & \mathbf{i} k \frac{\sqrt{n(n+1)}}{2 n+1} c_{n}^{0} a_{n}^{0}-\frac{\eta_{1}(n+1)}{2(2 n+1)} \frac{c_{n}^{1}(n+1) n}{\sqrt{n(n+1)}}\left(b_{n}^{1}+b_{n}^{-1}\right), \\
0= & \mathbf{i} k \frac{\sqrt{n(n+1)}}{2 n+1} c_{n}^{1}\left(a_{n}^{1}+a_{n}^{-1}\right)-\frac{\eta_{1}(n+1)}{2(2 n+1)} \frac{c_{n}^{2}}{\sqrt{n(n+1)}}(n+2)(n-1)\left(b_{n}^{2}+b_{n}^{-2}\right) \\
& +\frac{\eta_{1}(n+1)}{2 n+1} \frac{c_{n}^{0}}{\sqrt{n(n+1)}} b_{n}^{0}, \\
0= & \mathbf{i} k \frac{\sqrt{n(n+1)}}{2 n+1} c_{n}^{m}\left(a_{n}^{m}+a_{n}^{-m}\right)-\frac{\eta_{1}(n+1)}{2(2 n+1)} \frac{c_{n}^{m+1}}{\sqrt{n(n+1)}}(n+m+1)(n-m) \\
& \times\left(b_{n}^{m+1}+b_{n}^{-}(m+1)\right)+\frac{\eta_{1}(n+1)}{2(2 n+1)} \frac{c_{n}^{m-1}}{\sqrt{n(n+1)}}\left(b_{n}^{m-1}+b_{n}^{-(m-1)}\right), m=2,3, \ldots, n-1, \\
0= & \mathbf{i} k \frac{\sqrt{n(n+1)}}{2 n+1} c_{n}^{n}\left(a_{n}^{n}+a_{n}^{-n}\right)+\frac{\eta_{1}(n+1)}{2(2 n+1)} \frac{c_{n}^{n-1}}{\sqrt{n(n+1)}}\left(b_{n}^{n-1}+b_{n}^{-(n-1)}\right),
\end{aligned}\right.
$$


and

$$
\left\{\begin{aligned}
0= & \mathbf{i} k \frac{n+1}{2(2 n+1)} \frac{c_{n}^{1}}{\sqrt{n(n+1)}}(n+1) n\left(a_{n}^{1}+a_{n}^{-1}\right)+\eta_{1} \frac{c_{n}^{0} \sqrt{n(n+1)}}{2 n+1} b_{n}^{0}, \\
0= & \mathbf{i} k \frac{n+1}{2(2 n+1)} \frac{c_{n}^{2}}{\sqrt{n(n+1)}}(n+2)(n-1)\left(a_{n}^{2}+a_{n}^{-2}\right)-\mathbf{i} k \frac{n+1}{2 n+1} \frac{c_{n}^{0}}{\sqrt{n(n+1)}} a_{n}^{0} \\
& +\eta_{1} \frac{c_{n}^{1} \sqrt{n(n+1)}}{2 n+1}\left(b_{n}^{1}+b_{n}^{-1}\right), \\
0= & \mathbf{i} k \frac{n+1}{2(2 n+1)} \frac{c_{n}^{m}}{\sqrt{n(n+1)}}(n+m)(n-m+1)\left(a_{n}^{m}+a_{n}^{-m}\right)-\mathbf{i} k \cdot \frac{n+1}{2(2 n+1)} \frac{c_{n}^{m-2}}{\sqrt{n(n+1)}} \\
& \times\left(a_{n}^{m-2}+a_{n}^{-(m-2)}\right)+\eta_{1} \frac{c_{n}^{m-1} \sqrt{n(n+1)}}{2 n+1}\left(b_{n}^{m-1}+b_{n}^{-(m-1)}\right), \quad m=3,4, \ldots, n, \\
0= & -\mathbf{i} k \frac{n+1}{2(2 n+1)} \frac{c_{n}^{n-1}}{\sqrt{n(n+1)}}\left(a_{n}^{n-1}+a_{n}^{-(n-1)}\right)+\eta_{1} \frac{c_{n}^{n} \sqrt{n(n+1)}}{2 n+1}\left(b_{n}^{n}+b_{n}^{-n}\right)=0 .
\end{aligned}\right.
$$

Proof. Since the generalized impedance condition (1.3) associated with $\boldsymbol{\eta}_{1}$ is imposed on $\widetilde{\Pi}_{1}$, substituting 3.29 into 2.30 , by virtue of 2.10 , we derive that

$$
\begin{aligned}
\mathbf{0}= & \sum_{l=n}^{\infty} \sum_{m=-l}^{l} \frac{1}{\sqrt{l(l+1)}}\left\{\left(\mathbf{i} k a_{l}^{m} l(l+1) p_{l}(k r) c_{l}^{m} P_{l}^{m}+\boldsymbol{\eta}_{1} a_{l}^{m} j_{l}(k r) c_{l}^{m} \frac{\operatorname{sgn}(m)}{2}\left[P_{l-1}^{|m|+1}(\cos \theta)\right.\right.\right. \\
& \left.+(l+|m|-1)(l+|m|) P_{l-1}^{|m|-1}(\cos \theta)\right]-\boldsymbol{\eta}_{1} b_{l}^{m} q_{l}(k r) \frac{c_{l}^{m}}{2}[(l+|m|)(l-|m|+1) \\
& \left.\left.\times P_{l}^{|m|-1}(\cos \theta)-P_{l}^{|m|+1}(\cos \theta)\right]\right) \boldsymbol{e}_{\mathbf{1}}(\theta, 0)+\left(\mathbf { i } k b _ { l } ^ { m } j _ { l } ( k r ) c _ { l } ^ { m } \frac { \operatorname { s g n } ( m ) } { 2 } \left[P_{l-1}^{|m|+1}(\cos \theta)\right.\right. \\
& \left.+(l+|m|-1)(l+|m|) P_{l-1}^{|m|-1}(\cos \theta)\right]+\mathbf{i} k a_{l}^{m} q_{l}(k r) \frac{c_{l}^{m}}{2}[(l+|m|)(l-|m|+1) \\
& \left.\left.\left.\times P_{l}^{|m|-1}(\cos \theta)-P_{l}^{|m|+1}(\cos \theta)\right]+\boldsymbol{\eta}_{1} b_{l}^{m} l(l+1) p_{l}(k r) c_{l}^{m} P_{l}^{m}\right) \boldsymbol{e}_{\mathbf{2}}(\theta, 0)\right\}
\end{aligned}
$$

where $\boldsymbol{e}_{\mathbf{1}}(\theta, 0), \boldsymbol{e}_{\mathbf{2}}(\theta, 0) \boldsymbol{e}_{\mathbf{1}}\left(\theta, \phi_{0}\right)$ and $\boldsymbol{e}_{\mathbf{2}}\left(\theta, \phi_{0}\right)$ are defined in $(2.25)$.

Recall that $\boldsymbol{\eta}_{\ell}, \ell=1,2$, have the expansion $(3.1 \mathrm{a})$ and $\left.3.1 \mathrm{~b}\right)$ respectively, where the coefficients of $r^{0}$ in $(3.1 \mathrm{a})$ and $\sqrt{3.1 \mathrm{~b}}$ ) are non zero number $\eta_{1}$ and $\eta_{2}$. The lowest order term in 3.32 with respect to the power of $r$ is $r^{n-1}$, which is contributed by $p_{n}(k r)$ and $q_{n}(k r)$ from Remark 2.6. Furthermore, it is noted that coefficients of $r^{n-1}$ in $p_{n}(k r)$ and $q_{n}(k r)$ are $\frac{k^{n-1}}{(2 n+1)(2 n-1) ! !}$ and $\frac{(n+1) k^{n-1}}{(2 n+1)(2 n-1) ! !}$ respectively. Due to the fact that $\boldsymbol{e}_{1}(\theta, \phi)$ and $\boldsymbol{e}_{2}(\theta, \phi)$ are linearly independent for any $\theta$ and $\phi$, from Lemma 2.4, comparing the coefficient of $r^{n-1}$ both sides of 3.32 associated with $\boldsymbol{e}_{1}(\theta, \phi)$ for $\phi=0$, we have

$$
\begin{aligned}
0=\mathbf{i} k & \sum_{\substack{m=-n \\
m \neq 0}}^{n} a_{n}^{m} \frac{\sqrt{n(n+1)}}{2 n+1} c_{n}^{m} P_{n}^{m}(\cos \theta)-\eta_{1} \sum_{m=-n}^{n} b_{n}^{m} \cdot \frac{n+1}{2 n+1} \frac{1}{\sqrt{n(n+1)}} \frac{c_{n}^{m}}{2} \cdot((n+m) \\
& \left.\times(n-m+1) P_{n}^{m-1}(\cos \theta)-P_{n}^{m+1}(\cos \theta)\right)+\eta_{1} b_{n}^{0} \frac{n+1}{2 n+1} \frac{c_{n}^{0}}{\sqrt{n(n+1)}} P_{n}^{1}(\cos \theta),
\end{aligned}
$$


where for the index $m=0$ in $(3.32)$ we use the property 2.12$)$, and $c_{n}^{m}, m=0,1, \ldots, n$, are nonzero constants defined in (2.8). Utilizing the orthogonality condition (2.9), from (3.33) we can deduce (3.30).

Similarly, comparing the coefficient of $r^{n-1}$ both sides of $(3.32)$ associated with $\boldsymbol{e}_{2}(\theta, \phi)$ for $\phi=0$ in (3.32), from Lemma 2.4, we obtain the following $n+1$ equations:

$$
\begin{aligned}
0= & \mathbf{i} k \sum_{\substack{m=-n \\
m \neq 0}}^{n} a_{n}^{m} \cdot \frac{n+1}{2 n+1} \frac{1}{\sqrt{n(n+1)}} c_{n}^{m}\left((n+m)(n-m+1) P_{n}^{m-1}(\cos \theta)-P_{n}^{m+1}(\cos \theta)\right) \\
& -\mathbf{i} k a_{n}^{0} \cdot \frac{n+1}{2 n+1} \frac{1}{\sqrt{n(n+1)}} c_{n}^{0} P_{n}^{1}(\cos \theta)+\eta_{1} \sum_{m=-n}^{n} b_{n}^{m} \frac{\sqrt{n(n+1)}}{2 n+1} c_{n}^{m} P_{n}^{m}(\cos \theta),
\end{aligned}
$$

where for the index $m=0$ in $(3.32)$ we use the property $(2.12)$. By virtue of (3.34), utilizing the orthogonality condition (2.9), we can obtain (3.31).

Lemma 3.5. Under the same setup to Lemma 3.4 and assuming that there exists $n \in$ $\mathbb{N} \backslash\{1\}$ such that (3.29) is fulfilled, we have the following recursive linear equations:

$$
\begin{aligned}
0= & \mathbf{i} k \frac{\sqrt{n(n+1)}}{2 n+1} c_{n}^{0} a_{n}^{0}-\frac{\eta_{2}(n+1)}{2(2 n+1)} \frac{c_{n}^{1}(n+1) n}{\sqrt{n(n+1)}}\left(b_{n}^{1} e^{\mathbf{i} \alpha \cdot \pi}+b_{n}^{-1} e^{-\mathbf{i} \alpha \cdot \pi}\right), \\
0= & \mathbf{i} k \frac{\sqrt{n(n+1)}}{2 n+1} c_{n}^{1}\left(a_{n}^{1} e^{\mathbf{i} \alpha \cdot \pi}+a_{n}^{-1} e^{-\mathbf{i} \alpha \cdot \pi}\right)-\frac{\eta_{2}(n+1)}{2(2 n+1)} \frac{c_{n}^{2}(n+2)(n-1)}{\sqrt{n(n+1)}} \\
& \times\left(b_{n}^{2} e^{\mathbf{i} 2 \alpha \cdot \pi}+b_{n}^{-2} e^{-\mathbf{i} 2 \alpha \cdot \pi}\right)+\frac{\eta_{2}(n+1)}{2 n+1} \frac{c_{n}^{0}}{\sqrt{n(n+1)}} b_{n}^{0}, \\
0= & \mathbf{i} k \frac{\sqrt{n(n+1)}}{2 n+1} c_{n}^{m}\left(a_{n}^{m} e^{\mathbf{i} m \alpha \cdot \pi}+a_{n}^{-m} e^{-\mathbf{i} m \alpha \cdot \pi}\right)-\frac{\eta_{2}(n+1)}{2(2 n+1)} \frac{c_{n}^{m+1}}{\sqrt{n(n+1)}}(n+m+1) \\
& \times(n-m)\left(b_{n}^{m+1} e^{\mathbf{i}(m+1) \alpha \cdot \pi}+b_{n}^{-(m+1)} e^{-\mathbf{i}(m+1) \alpha \cdot \pi}\right)+\frac{\eta_{2}(n+1)}{2(2 n+1)} \frac{c_{n}^{m-1}}{\sqrt{n(n+1)}} \\
& \times\left(b_{n}^{m-1} e^{\mathbf{i}(m-1) \alpha \cdot \pi}+b_{n}^{-(m-1)} e^{-\mathbf{i}(m-1) \alpha \cdot \pi}\right), \quad m=2,3, \ldots, n-1, \\
0= & \mathbf{i} k \frac{\sqrt{n(n+1)}}{2 n+1} c_{n}^{n}\left(a_{n}^{n} e^{\mathbf{i} n \alpha \cdot \pi}+a_{n}^{-n} e^{-\mathbf{i} n \alpha \cdot \pi}\right)+\frac{\eta_{2}(n+1)}{2(2 n+1)} \frac{c_{n}^{n-1}}{\sqrt{n(n+1)}} \\
& \times\left(b_{n}^{n-1} e^{\mathbf{i}(n-1) \alpha \pi}+b_{n}^{-(n-1)} e^{-\mathbf{i}(n-1) \alpha \pi}\right),
\end{aligned}
$$

and

$$
\begin{aligned}
0= & \mathbf{i} k \frac{n+1}{2(2 n+1)} \frac{c_{n}^{1}}{\sqrt{n(n+1)}}(n+1) n\left(a_{n}^{1} e^{\mathbf{i} \alpha \cdot \pi}+a_{n}^{-1} e^{-\mathbf{i} \alpha \cdot \pi}\right)+\eta_{2} \frac{c_{n}^{0} \sqrt{n(n+1)}}{2 n+1} b_{n}^{0}, \\
0= & \mathbf{i} k \frac{n+1}{2(2 n+1)} \frac{c_{n}^{2}}{\sqrt{n(n+1)}}(n+2)(n-1)\left(a_{n}^{2} e^{2 \mathbf{i} \alpha \cdot \pi}+a_{n}^{-2} e^{-2 \mathbf{i} \alpha \cdot \pi}\right)-\mathbf{i} k \frac{n+1}{2 n+1} \\
& \times \frac{c_{n}^{0}}{\sqrt{n(n+1)}} a_{n}^{0}+\eta_{2} \frac{c_{n}^{1} \sqrt{n(n+1)}}{2 n+1}\left(b_{n}^{1} e^{\mathbf{i} \alpha \cdot \pi}+b_{n}^{-1} e^{-\mathbf{i} \alpha \cdot \pi}\right), \\
0= & \mathbf{i} k \frac{n+1}{2(2 n+1)} \frac{c_{n}^{m}(n+m)(n-m+1)}{\sqrt{n(n+1)}}\left(a_{n}^{m} e^{\mathbf{i} m \alpha \cdot \pi}+a_{n}^{-m} e^{-\mathbf{i} m \alpha \cdot \pi}\right)-\mathbf{i} k \cdot \frac{n+1}{2(2 n+1)} \\
& \times \frac{c_{n}^{m-2}}{\sqrt{n(n+1)}}\left(a_{n}^{m-2} e^{\mathbf{i}(m-2) \alpha \cdot \pi}+a_{n}^{-(m-2)} e^{-\mathbf{i}(m-2) \alpha \cdot \pi}\right)+\eta_{2} \frac{c_{n}^{m-1} \sqrt{n(n+1)}}{2 n+1} \\
& \times\left(b_{n}^{m-1} e^{\mathbf{i}(m-1) \alpha \cdot \pi}+b_{n}^{-(m-1)} e^{-\mathbf{i}(m-1) \alpha \cdot \pi}\right), \quad m=3,4, \ldots, n,
\end{aligned}
$$




$$
\begin{aligned}
0= & -\mathbf{i} k \frac{n+1}{2(2 n+1)} \frac{c_{n}^{n-1}}{\sqrt{n(n+1)}}\left(a_{n}^{n-1} e^{\mathbf{i}(n-1) \alpha \cdot \pi}+a_{n}^{-(n-1)} e^{-\mathbf{i}(n-1) \alpha \cdot \pi}\right)+\eta_{2} \frac{c_{n}^{n} \sqrt{n(n+1)}}{2 n+1} \\
& \times\left(b_{n}^{n} e^{\mathbf{i} n \alpha \cdot \pi}+b_{n}^{-n} e^{-\mathbf{i} n \alpha \cdot \pi}\right)=0 .
\end{aligned}
$$

Proof. Since the generalized impedance condition (1.3) associated with $\boldsymbol{\eta}_{2}$ is imposed on $\widetilde{\Pi}_{2}$, substituting 3.29 into 2.31 , by virtue of 2.10 , we derive that

$$
\begin{aligned}
\mathbf{0}= & \sum_{l=n}^{\infty} \sum_{m=-l}^{l} \frac{e^{\mathbf{i} m \alpha \cdot \pi}}{\sqrt{l(l+1)}}\left\{\left(\mathbf{i} k a_{l}^{m} l(l+1) p_{l}(k r) c_{l}^{m} P_{l}^{m}+\boldsymbol{\eta}_{2} a_{l}^{m} j_{l}(k r) c_{l}^{m} \frac{\operatorname{sgn}(m)}{2}\left[P_{l-1}^{|m|+1}(\cos \theta)\right.\right.\right. \\
& \left.+(l+|m|-1)(l+|m|) P_{l-1}^{|m|-1}(\cos \theta)\right]-\boldsymbol{\eta}_{2} b_{l}^{m} q_{l}(k r) \frac{c_{l}^{m}}{2}[(l+|m|)(l-|m|+1) \\
& \left.\left.\times P_{l}^{|m|-1}(\cos \theta)-P_{l}^{|m|+1}(\cos \theta)\right]\right) \boldsymbol{e}_{\mathbf{1}}\left(\theta, \phi_{0}\right)+\left(\mathbf { i } k b _ { l } ^ { m } j _ { l } ( k r ) c _ { l } ^ { m } \frac { \operatorname { s g n } ( m ) } { 2 } \left[P_{l-1}^{|m|+1}(\cos \theta)\right.\right. \\
& \left.+(l+|m|-1)(l+|m|) P_{l-1}^{|m|-1}(\cos \theta)\right]+\mathbf{i} k a_{l}^{m} q_{l}(k r) \frac{c_{l}^{m}}{2}[(l+|m|)(l-|m|+1) \\
& \left.\left.\left.\times P_{l}^{|m|-1}(\cos \theta)-P_{l}^{|m|+1}(\cos \theta)\right]+\boldsymbol{\eta}_{2} b_{l}^{m} l(l+1) p_{l}(k r) c_{l}^{m} P_{l}^{m}\right) \boldsymbol{e}_{\mathbf{2}}\left(\theta, \phi_{0}\right)\right\},
\end{aligned}
$$

where $\boldsymbol{e}_{\mathbf{1}}(\theta, 0), \boldsymbol{e}_{\mathbf{2}}(\theta, 0) \boldsymbol{e}_{\mathbf{1}}\left(\theta, \phi_{0}\right)$ and $\boldsymbol{e}_{\mathbf{2}}\left(\theta, \phi_{0}\right)$ are defined in $(2.25)$.

Recall that $\boldsymbol{\eta}_{\ell}, \ell=1,2$, have the expansion (3.1a) and (3.1b) respectively, where the coefficients of $r^{0}$ in $\left.3.1 \mathrm{a}\right)$ and $\left(3.1 \mathrm{~b}\right.$ are non zero number $\eta_{1}$ and $\eta_{2}$. Comparing the coefficient of $r^{n-1}$ both sides of (3.37) associated with $\boldsymbol{e}_{1}(\theta, \phi)$ and $\boldsymbol{e}_{2}(\theta, \phi)$ for $\phi=\phi_{0}$ respectively, utilizing the orthogonality condition $(2.9)$, we can derive (3.35) and (3.36).

The next theorem characterises the vanishing order of $\mathbf{E}$ to 1.1$)$ at $\mathbf{0} \in \mathcal{E}\left(\widetilde{\Pi}_{1}, \widetilde{\Pi}_{2}, \boldsymbol{l}\right)$ with $\boldsymbol{\eta}_{j} \in \mathcal{A}(\boldsymbol{l})$.

Theorem 3.6. Let $\mathbf{E}$ be a a solution to 1.1$)$, whose radial wave expansion in $B_{\rho_{0}}(\mathbf{0})$ is given by 2.17). Consider a generalized impedance edge-corner $\mathcal{E}\left(\widetilde{\Pi}_{1}, \widetilde{\Pi}_{2}, \boldsymbol{l}\right) \Subset \Omega$ with $\angle\left(\Pi_{1}, \Pi_{2}\right)=\phi_{0}=\alpha \pi$, where $\alpha \in(0,2)$ and $\alpha \neq 1$. Suppose that the generalized impedance parameters $\boldsymbol{\eta}_{j}$ on $\widetilde{\Pi}_{j}, j=1,2$, are given by 3.1a and 3.1b respectively. Then it holds that $\mathbf{E}$ vanishes up to the order $N$ at $\mathbf{0}$ :

$\operatorname{Vani}(\mathbf{E} ; \mathbf{0}) \geq\left\{\begin{array}{l}1, \quad \text { if } \alpha \neq \frac{1}{2}, \\ N \in \mathbb{N} \backslash\{1\}, \text { if } \alpha \neq \frac{q}{p}, p=1, \ldots, N, \text { and for a fixed } p, q=1, \ldots, 2 p-1 .\end{array}\right.$

Proof. We prove this theorem by induction. Assume that

$$
\alpha \neq \frac{1}{2} \text { and } \alpha \neq \frac{3}{2} \text {. }
$$

Since the generalized impedance condition $(1.3)$ associated with $\boldsymbol{\eta}_{1}$ is imposed on $\widetilde{\Pi}_{1}$, it yields 3.32 when the summation index $n=1$. Comparing the coefficient of $r^{0}$ associated with $\boldsymbol{e}_{\mathbf{2}}(\theta, 0)$ on both sides of 3.32 for the case that the summation index $n=1$, from Lemma 2.3 , we can obtain that

$$
0=\mathbf{i} k \frac{4 c_{1}^{1}}{6 \sqrt{2}}\left(a_{1}^{1}+a_{1}^{-1}\right)+\eta_{1} \frac{\sqrt{2} c_{1}^{0}}{3} b_{1}^{0} .
$$


Combine $(3.39)$ with $(3.2 \mathrm{a})$ and $(3.2 \mathrm{~b})$ from Lemma 3.3 , we derive that

$$
\mathcal{A}_{1}\left[\begin{array}{c}
a_{1}^{1}+a_{1}^{-1} \\
a_{1}^{1}-a_{1}^{-1} \\
b_{1}^{0}
\end{array}\right]=\mathbf{0}, \quad \mathcal{A}_{1}=\left(\alpha_{i j}^{1}\right)_{i, j=1}^{3}
$$

where

$$
\begin{aligned}
& \alpha_{11}=\frac{4 \mathbf{i} k c_{1}^{1} \sin ^{2} \phi_{0}}{6 \sqrt{2}}, \quad \alpha_{12}=-\frac{4 k c_{1}^{1} \sin \phi_{0} \cos \phi_{0}}{6 \sqrt{2}}, \quad \alpha_{13}=\frac{\left(-\eta_{2} \cos \phi_{0}-\eta_{1}\right) \sqrt{2} c_{1}^{0}}{3} \\
& \alpha_{21}=-\frac{4 \mathbf{i} k c_{1}^{1} \sin \phi_{0} \cos \phi_{0}}{6 \sqrt{2}}, \quad \alpha_{22}=-\frac{4 k c_{1}^{1} \sin ^{2} \phi_{0}}{6 \sqrt{2}}, \quad \alpha_{23}=-\frac{\eta_{2} \sqrt{2} c_{1}^{0} \sin \phi_{0}}{3} \\
& \alpha_{31}=\frac{4 \mathbf{i} k c_{1}^{1}}{6 \sqrt{2}}, \quad \alpha_{32}=0, \quad \alpha_{33}=\frac{\sqrt{2} c_{1}^{0} \eta_{1}}{3} .
\end{aligned}
$$

By direct calculations, it yields that

$$
\left|\mathcal{A}_{1}\right|=-\mathbf{i} k^{2} \eta_{1}\left(\frac{2}{3}\right)^{3} \frac{\sqrt{2}}{2}\left(c_{1}^{1}\right)^{2} c_{1}^{0} \sin ^{2}(\alpha \pi)
$$

Hence under the assumption $(3.38)$ and $\eta_{1} \neq 0$, by virtue of 3.40$)$ and $k \in \mathbb{R}_{+}$, it can be derived that $a_{1}^{ \pm 1}=b_{1}^{0}=0$. Recall that (3.24) is given by Lemma 3.3. In view of 3.38), $\alpha \in(0,1), k \in \mathbb{R}_{+}$and $\eta_{2} \neq 0$, using the fact that

$$
\left|\mathcal{B}_{1}\right|=-k \eta_{2}^{2}\left(\frac{2}{3}\right)^{3} \frac{\sqrt{2}}{2}\left(c_{1}^{1}\right)^{2} c_{1}^{0} \sin ^{2}(\alpha \pi) \cos ^{2}(\alpha \pi) \neq 0,
$$

where $\mathcal{B}_{1}$ is defined in 3.24 , we can obtain that $b_{1}^{ \pm 1}=a_{1}^{0}=0$. Therefore, from Lemma 2.8 . we prove that $\operatorname{Vani}(\mathbf{E} ; \mathbf{0}) \geq 1$ under conditions $(3.38)$ and $\eta_{\ell} \neq 0, \ell=1,2$.

Suppose that $N=2$, from the assumption in this theorem we know that $(3.38)$ still holds. Since $a_{1}^{ \pm 1}=b_{1}^{ \pm 1}=a_{1}^{0}=b_{1}^{0}=0$, from Lemmas 3.4 and 3.5 we know that (3.30), (3.31), 3.35) and (3.36) for $n=2$ hold. Therefore we have

$$
\left\{\begin{array}{l}
0=\frac{\sqrt{6} c_{2}^{0} \mathbf{i} k}{5} a_{2}^{0}-\frac{18 c_{2}^{1} \eta_{1}}{10 \sqrt{6}}\left(b_{2}^{1}+b_{2}^{-1}\right), \\
0=\frac{\sqrt{6} c_{2}^{1} \mathbf{i} k}{5}\left(a_{2}^{1}+a_{2}^{-1}\right)-\frac{12 c_{2}^{2} \eta_{1}}{10 \sqrt{6}}\left(b_{2}^{2}+b_{2}^{-2}\right)+\frac{3 c_{2}^{0} \eta_{1}}{5 \sqrt{6}} b_{2}^{0} \\
0=\mathbf{i} k \frac{\sqrt{6}}{5} c_{2}^{2}\left(a_{2}^{2}+a_{2}^{-2}\right)+\frac{3 c_{2}^{1} \eta_{1}}{10 \sqrt{6}}\left(b_{2}^{1}+b_{2}^{-1}\right),
\end{array}\right.
$$

and

$$
\left\{\begin{array}{l}
0=\frac{18 c_{2}^{1} \mathbf{i} k}{10 \sqrt{6}}\left(a_{2}^{1}+a_{2}^{-1}\right)+\frac{\sqrt{6} c_{2}^{0} \eta_{1}}{5} b_{2}^{0}, \\
0=\frac{12 c_{2}^{2} \mathbf{i} k}{10 \sqrt{6}}\left(a_{2}^{2}+a_{2}^{-2}\right)-\frac{3 c_{2}^{0} \mathbf{i} k}{5 \sqrt{6}} a_{2}^{0}+\frac{\sqrt{6} c_{2}^{1} \eta_{1}}{5}\left(b_{2}^{1}+b_{2}^{-1}\right), \\
0=-\frac{3 c_{2}^{1} \mathbf{i} k}{10 \sqrt{6}}\left(a_{2}^{1}+a_{2}^{-1}\right)+\frac{\sqrt{6} c_{2}^{2} \eta_{1}}{5}\left(b_{2}^{2}+b_{2}^{-2}\right)=0 .
\end{array}\right.
$$


Furthermore, it holds that

$$
\begin{aligned}
& \left\{\begin{array}{l}
0=\frac{\sqrt{6} c_{2}^{0} \mathbf{i} k}{5} a_{2}^{0}-\frac{18 c_{2}^{1} \eta_{2}}{10 \sqrt{6}}\left(b_{2}^{1} e^{\mathbf{i} \alpha \cdot \pi}+b_{2}^{-1} e^{-\mathbf{i} \alpha \cdot \pi}\right), \\
0=\frac{\sqrt{6} c_{2}^{1} \mathbf{i} k}{5}\left(a_{2}^{1} e^{\mathbf{i} \alpha \cdot \pi}+a_{2}^{-1} e^{-\mathbf{i} \alpha \cdot \pi}\right)-\frac{12 c_{2}^{2} \eta_{2}}{10 \sqrt{6}}\left(b_{2}^{2} e^{\mathbf{i} 2 \alpha \cdot \pi}+b_{2}^{-2} e^{-\mathbf{i} 2 \alpha \cdot \pi}\right)+\frac{3 c_{2}^{0} \eta_{2}}{5 \sqrt{6}} b_{2}^{0}, \\
0=\frac{\sqrt{6} c_{2}^{2} \mathbf{i} k}{5}\left(a_{2}^{2} e^{\mathbf{i} 2 \alpha \cdot \pi}+a_{2}^{-2} e^{-\mathbf{i} 2 \alpha \cdot \pi}\right)+\frac{3 c_{2}^{1} \eta_{2}}{10 \sqrt{6}}\left(b_{2}^{1} e^{\mathbf{i} \alpha \pi}+b_{2}^{-1} e^{-\mathbf{i} \alpha \pi}\right),
\end{array}\right. \\
& \left\{\begin{array}{l}
0=\frac{18 c_{2}^{1} \mathbf{i} k}{10 \sqrt{6}}\left(a_{2}^{1} e^{\mathbf{i} \alpha \cdot \pi}+a_{2}^{-1} e^{-\mathbf{i} \alpha \cdot \pi}\right)+\frac{\sqrt{6} c_{2}^{0} \eta_{2}}{5} b_{2}^{0}, \\
0=\frac{12 c_{2}^{2} \mathbf{i} k}{10 \sqrt{6}}\left(a_{2}^{2} e^{2 \mathbf{i} \alpha \cdot \pi}+a_{2}^{-2} e^{-2 \mathbf{i} \alpha \cdot \pi}\right)-\frac{3 c_{2}^{0} \mathbf{i} k}{5 \sqrt{6}} a_{2}^{0}+\frac{\sqrt{6} c_{2}^{1} \eta_{2}}{5}\left(b_{2}^{1} e^{\mathbf{i} \alpha \cdot \pi}+b_{2}^{-1} e^{-\mathbf{i} \alpha \cdot \pi}\right), \\
0=-\frac{3 c_{2}^{1} \mathbf{i} k}{10 \sqrt{6}}\left(a_{2}^{1} e^{\mathbf{i} \alpha \cdot \pi}+a_{2}^{-1} e^{-\mathbf{i} \alpha \cdot \pi}\right)+\frac{\sqrt{6} c_{2}^{2} \eta_{2}}{5}\left(b_{2}^{2} e^{\mathbf{i} 2 \alpha \cdot \pi}+b_{2}^{-2} e^{-\mathbf{i} 2 \alpha \cdot \pi}\right)=0 .
\end{array}\right.
\end{aligned}
$$

From Lemma 3.1, (3.4a) and (3.4b) for $n=2$ can be written as

$$
\begin{aligned}
& 0=\frac{18 c_{2}^{1} \sin ^{2} \phi_{0} \mathbf{i} k}{10 \sqrt{6}}\left(a_{2}^{1}+a_{2}^{-1}\right)-\frac{18 c_{2}^{1} \sin \phi_{0} \cos \phi_{0} k}{10 \sqrt{6}}\left(a_{2}^{1}-a_{2}^{-1}\right)+\frac{\sqrt{6} c_{2}^{0}\left(-\eta_{2} \cos \phi_{0}-\eta_{1}\right)}{5} b_{2}^{0}, \\
& 0=-\frac{18 c_{2}^{1} \sin \phi_{0} \cos \phi_{0} \mathbf{i} k}{10 \sqrt{6}}\left(a_{2}^{1}+a_{2}^{-1}\right)-\frac{18 c_{2}^{1} \sin ^{2} \phi_{0} k}{10 \sqrt{6}}\left(a_{2}^{1}-a_{2}^{-1}\right)-\frac{\eta_{2} \sqrt{6} c_{2}^{0} \sin \phi_{0}}{5} b_{2}^{0} .
\end{aligned}
$$

Combing the first equation of 3.42 with 3.45 , we have

$$
\mathcal{A}_{2}\left[\begin{array}{c}
a_{2}^{1}+a_{2}^{-1} \\
a_{2}^{1}-a_{2}^{-1} \\
b_{2}^{0}
\end{array}\right]=\mathbf{0}, \quad \mathcal{A}_{2}=\left(\alpha_{i j}^{2}\right)_{i, j=1}^{3},
$$

where

$$
\begin{aligned}
& \alpha_{11}^{2}=\frac{18 c_{2}^{1} \sin ^{2} \phi_{0} \mathbf{i} k}{10 \sqrt{6}}, \quad \alpha_{12}^{2}=-\frac{18 c_{2}^{1} \sin \phi_{0} \cos \phi_{0} k}{10 \sqrt{6}}, \quad \alpha_{13}^{2}=\frac{\sqrt{6} c_{2}^{0}\left(-\eta_{2} \cos \phi_{0}-\eta_{1}\right)}{5} \\
& \alpha_{21}^{2}=-\frac{18 c_{2}^{1} \sin \phi_{0} \cos \phi_{0} \mathbf{i} k}{10 \sqrt{6}}, \quad \alpha_{22}^{2}=-\frac{18 c_{2}^{1} \sin ^{2} \phi_{0} k}{10 \sqrt{6}}, \quad \alpha_{23}^{2}=-\frac{\eta_{2} \sqrt{6} c_{2}^{0} \sin \phi_{0}}{5} \\
& \alpha_{31}^{2}=\frac{18 c_{2}^{1} \mathbf{i} k}{10 \sqrt{6}}, \quad \alpha_{32}^{2}=0, \quad \alpha_{33}^{2}=\frac{\sqrt{6} c_{2}^{0} \eta_{1}}{5} .
\end{aligned}
$$

It can be computed directly that

$$
\left|\mathcal{A}_{2}\right|=-\mathbf{i} k^{2} \eta_{1}\left(\frac{3}{5}\right)^{3} \frac{2 \sqrt{6}}{2}\left(c_{2}^{1}\right)^{2} c_{2}^{0} \sin ^{2}(\alpha \pi) .
$$

Since $\alpha \in(0,2), \alpha \neq 1, \eta_{1} \neq 0$ and $k \in \mathbb{R}_{+}$, in view of $(3.46)$, we prove that $a_{2}^{ \pm 1}=b_{2}^{0}=0$.

Recall that $\mathbf{E}$ has the radial wave expansion (2.17) at $\mathbf{0}$. Due to that $\eta_{1} \neq 0$, under the assumption (3.38), by virtue of (3.27) in Lemma 3.3, we have

$$
a_{1}^{0}=b_{1}^{ \pm 1}=0 .
$$

By mathematical induction, if $\alpha \neq \frac{q}{p}$, where $p=1, \ldots, n-1$ and for a fixed $p$, $q=1,2, \ldots, 2 p-1$, then

$$
\operatorname{Vani}(\mathbf{E} ; \mathbf{0}) \geq n-1 \text {. }
$$


From Lemma 2.8, we know that

$$
a_{l}^{m}=b_{l}^{m}=0, \quad m \in[l]_{0}, \quad l=1,2, \ldots, n .
$$

Therefore we know that (3.30), (3.31), (3.35) and (3.36) hold from Lemmas 3.4 and 3.5 . In the following under the assumption

$$
\eta_{\ell} \neq 0 \text { for } \ell=1,2 \text { and } \alpha \neq \frac{q}{p}, p=1, \ldots, n,
$$

where for a fixed $p, q=1,2, \ldots, 2 p-1$, we shall show that

$$
a_{n}^{m}=b_{n}^{m}=0, \quad \forall m \in[n]_{0}
$$

by utilizing the recursive equations of (3.30), (3.31), (3.35) and (3.36). Indeed, combing the first equation of (3.31) with (3.2a) and $(3.2 \mathrm{~b})$, we have

$$
\mathcal{A}_{n}\left[\begin{array}{c}
a_{n}^{1}+a_{n}^{-1} \\
a_{n}^{1}-a_{n}^{-1} \\
b_{n}^{0}
\end{array}\right]=\mathbf{0}, \quad \mathcal{A}_{n}=\left(\alpha_{i j}^{n}\right)_{i, j=1}^{3}
$$

where

$$
\begin{aligned}
& \alpha_{11}^{n}=\frac{\mathbf{i} k n(n+1)^{2} c_{n}^{1} \sin ^{2} \phi_{0}}{2(2 n+1) \sqrt{n(n+1)}}, \quad \alpha_{12}^{n}=-\frac{k n(n+1)^{2} c_{n}^{1} \sin \phi_{0} \cos \phi_{0}}{2(2 n+1) \sqrt{n(n+1)}} \\
& \alpha_{13}^{n}=\frac{\left(-\eta_{2} \cos \phi_{0}-\eta_{1}\right) \sqrt{n(n+1)} c_{n}^{0}}{2 n+1}, \quad \alpha_{21}^{n}=-\frac{\mathbf{i} k n(n+1)^{2} c_{n}^{1} \sin \phi_{0} \cos \phi_{0}}{2(2 n+1) \sqrt{n(n+1)}} \\
& \alpha_{22}^{n}=-\frac{k n(n+1)^{2} c_{n}^{1} \sin ^{2} \phi_{0}}{2(2 n+1) \sqrt{n(n+1)}}, \quad \alpha_{23}^{n}=-\frac{\eta_{2} \sqrt{n(n+1)} c_{n}^{0} \sin \phi_{0}}{2 n+1} \\
& \alpha_{31}^{n}=\mathbf{i} k \frac{n(n+1)^{2} c_{n}^{1}}{2(2 n+1) \sqrt{n(n+1)}}, \quad \alpha_{32}^{n}=0, \quad \alpha_{33}^{n}=\eta_{1} \frac{\sqrt{n(n+1)} c_{n}^{0}}{2 n+1}
\end{aligned}
$$

It can be derived that

$$
\left|\mathcal{A}_{n}\right|=-\mathbf{i} k^{2} \eta_{1}\left(\frac{n+1}{2 n+1}\right)^{3} \frac{n \sqrt{n(n+1)}}{2}\left(c_{n}^{1}\right)^{2} c_{n}^{0} \sin ^{2}(\alpha \pi) .
$$

Since $\alpha \in(0,2), \alpha \neq 1, \alpha \neq \frac{1}{2}, \alpha \neq \frac{3}{2}$ and $\eta_{\ell} \neq 0, \ell=1,2$, by virtue of $(3.52),(3.53)$ and Lemma 3.3 , we have

$$
a_{n}^{ \pm 1}=a_{n}^{0}=b_{n}^{ \pm 1}=b_{n}^{0}=0 .
$$

Substituting (3.54) into the second equation of (3.30), (3.31), 3.35) and (3.36), since $k \in \mathbb{R}_{+}, \eta_{\ell} \neq 0$ for $\ell=1,2$ and $c_{n}^{2} \neq 0$, we obtain that

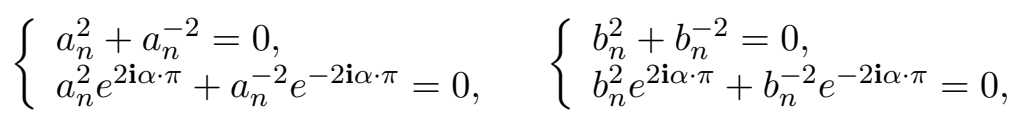

which can be shown to prove that $a_{n}^{ \pm 2}=b_{n}^{ \pm 2}=0$, since

$$
\left|\begin{array}{cc}
1 & 1 \\
e^{\mathbf{i} 2 \alpha \cdot \pi} & e^{-\mathbf{i} 2 \alpha \cdot \pi}
\end{array}\right|=-2 \mathbf{i} \sin (2 \alpha \pi) \neq 0,
$$

under (3.50). Substituting

$$
a_{n}^{ \pm 1}=b_{n}^{ \pm 1}=a_{n}^{ \pm 2}=b_{n}^{ \pm 2}=0
$$

into the third equation of (3.30), (3.31), (3.35) and (3.36), since $k \in \mathbb{R}_{+}, \eta_{\ell} \neq 0$ for $\ell=1,2$ and $c_{n}^{3} \neq 0$, we get that

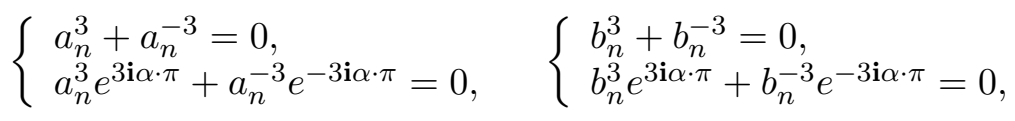


which can be shown to prove that $a_{n}^{ \pm 3}=b_{n}^{ \pm 3}=0$, since

$$
\left|\begin{array}{cc}
1 & 1 \\
e^{\mathbf{i} 3 \alpha \cdot \pi} & e^{-\mathbf{i} 3 \alpha \cdot \pi}
\end{array}\right|=-2 \mathbf{i} \sin (3 \alpha \pi) \neq 0,
$$

under (3.50). Repeating the above procedures step by step, utilizing the recursive property of (3.30), (3.31), 3.35) and (3.36), we can prove (3.51). Generally, assume that we have proved that

$$
a_{n}^{ \pm m}=b_{n}^{ \pm m}=0 \text { for } m=0,1, \ldots, \ell .
$$

Substituting $a_{n}^{ \pm(\ell-1)}=b_{n}^{ \pm(\ell-2)}=0$ into the $\ell$-th equation of 3.30 and 3.35, we can obtain that

$$
\left\{\begin{array}{l}
b_{n}^{\ell}+b_{n}^{-\ell}=0, \\
b_{n}^{\ell} e^{\mathbf{i} \ell \cdot \cdot \pi}+b_{n}^{-\ell} e^{-\mathbf{i} \ell \alpha \cdot \pi}=0,
\end{array}\right.
$$

under the assumption $\eta_{1} \neq 0$ and $\eta_{2} \neq 0$. Substituting $a_{n}^{ \pm(\ell-2)}=b_{n}^{ \pm(\ell-1)}=0$ into the $\ell$-th equation of (3.31) and (3.36), we can get that

$$
\left\{\begin{array}{l}
a_{n}^{\ell}+a_{n}^{-\ell}=0 \\
a_{n}^{\ell} e^{\mathrm{i} \ell \cdot \pi}+a_{n}^{-2} e^{-\mathbf{i} \ell \alpha \cdot \pi}=0,
\end{array}\right.
$$

Hence from 3.55 and $(3.56)$, under 3.50$)$ it yields that $a_{n}^{ \pm \ell}=b_{n}^{ \pm \ell}=0$.

Therefore, due to (3.51), by virtue of Lemma 2.8 , we prove that

$$
\operatorname{Vani}(\mathbf{E} ; \mathbf{0}) \geq n,
$$

which completes the proof of this theorem.

4. VANishing ORDERs FOR AN EDGE-CORNER $\mathcal{E}\left(\widetilde{\Pi}_{1}, \widetilde{\Pi}_{2}, \boldsymbol{l}\right)$ With $\boldsymbol{\eta}_{j} \in \mathcal{A}(\boldsymbol{l})$ OR

$$
\boldsymbol{\eta}_{j}=0, \infty
$$

In this section, we investigate the vanishing order of the solution $\mathbf{E}$ to $(1.1)$ at an edgecorner point $\mathbf{0} \in \mathcal{E}\left(\widetilde{\Pi}_{1}, \widetilde{\Pi}_{2}, \boldsymbol{l}\right)$, where the generalized impedance edge-corner $\mathcal{E}\left(\widetilde{\Pi}_{1}, \widetilde{\Pi}_{2}, \boldsymbol{l}\right) \Subset$ $\Omega$ with $\angle\left(\Pi_{1}, \Pi_{2}\right)=\phi_{0}=\alpha \pi, \alpha \in(0,2)$ and $\alpha \neq 1$. The generalized impedance condition 1.3 on $\widetilde{\Pi}_{j}, j=1,2$, are different. Namely, the associated generalized impedance parameter of the generalized impedance edge-corner $\mathcal{E}\left(\Pi_{1}, \Pi_{2}, \boldsymbol{l}\right)$ in Theorem 4.2 are $\boldsymbol{\eta}_{1} \equiv \infty$ and $\boldsymbol{\eta}_{2} \equiv 0$, where we utilize Lemma 4.1 to reveal the vanishing order of $\mathbf{E}$ at $\mathbf{0}$. On the other hand, in Theorems 4.4 and 4.5 , we consider the case that $\boldsymbol{\eta}_{2} \in \mathcal{A}(\boldsymbol{l})$ has the expansion $(3.1 \mathrm{~b})$ whereas the associated generalized impedance parameter $\boldsymbol{\eta}_{1}$ could be either $\infty$ or 0 . The reflection principle [13,14] are adopted to transform the corresponding generalized impedance edge-corner to be generalized impedance edge-corner intersected by two plane cells with the generalized impedance condition 1.3 and two associated generalized impedance parameters belonging to $\mathcal{A}(\boldsymbol{l})$.

Lemma 4.1. Let $\mathbf{E}$ be a a solution to (1.1), whose radial wave expansion in $B_{\rho_{0}}(\mathbf{0})$ is given by 2.17). Consider a generalized impedance edge-corner $\mathcal{E}\left(\widetilde{\Pi}_{1}, \widetilde{\Pi}_{2}, \boldsymbol{l}\right) \Subset \Omega$ with $\angle\left(\Pi_{1}, \Pi_{2}\right)=\phi_{0}=\alpha \pi$, where $\alpha \in(0,2)$ and $\alpha \neq 1$. Suppose that the generalize impedance parameters $\boldsymbol{\eta}_{1}$ on $\widetilde{\Pi}_{1}$ satisfies (ii) in (1.2) and $\boldsymbol{\eta}_{2}$ on $\widetilde{\Pi}_{2}$ satisfies (i) in (1.2) respectively. It holds that

$$
\begin{aligned}
& b_{1}^{1}+b_{1}^{-1}=0, \quad b_{1}^{0}=0, \\
& a_{1}^{1}-a_{1}^{-1}=0, \\
& b_{2}^{m}+b_{2}^{-m}=0, \quad m=1,2, \quad \text { and } b_{2}^{0}=0,
\end{aligned}
$$

and

$$
a_{1}^{1} e^{\mathbf{i} \alpha \cdot \pi}+a_{1}^{-1} e^{-\mathbf{i} \alpha \cdot \pi}=0, \quad a_{1}^{0}=0
$$




$$
\begin{aligned}
& b_{1}^{1} e^{\mathbf{i} \alpha \cdot \pi}-b_{1}^{-1} e^{-\mathbf{i} \alpha \cdot \pi}=0, \\
& a_{2}^{m} e^{\mathbf{i} m \alpha \cdot \pi}+a_{2}^{-m} e^{-\mathbf{i} m \alpha \cdot \pi}=0, \quad m=1,2, \text { and } a_{2}^{0}=0 .
\end{aligned}
$$

Assume that there exits a $n \in \mathbb{N}$ such that

$$
a_{l}^{m}=b_{l}^{m}=0, \quad l=1,2, \ldots, n-1, \quad m \in[l]_{0},
$$

then we have

$$
\begin{aligned}
& b_{n}^{m}+b_{n}^{-m}=0, \quad m=1, \ldots, n, \text { and } b_{n}^{0}=0, \\
& a_{n}^{m} e^{\mathbf{i} m \alpha \cdot \pi}+a_{n}^{-m} e^{-\mathbf{i} m \alpha \cdot \pi}=0, \quad m=1, \ldots, n, \text { and } a_{n}^{0}=0,
\end{aligned}
$$

and

$$
\begin{aligned}
& \sum_{m=1}^{n} m c_{n}^{m}\left(a_{n}^{m}-a_{n}^{-m}\right) \frac{P_{n}^{m}(\cos \theta)}{\sin \theta}+\left.\sum_{m=-(n+1)}^{n+1} \frac{c_{n+1}^{m}(n+2)}{2 n+3} b_{n+1}^{m} \frac{\partial Y_{n+1}^{m}}{\partial \theta}\right|_{\phi=0}=0 \\
& \sum_{m=1}^{n} m c_{n}^{m}\left(b_{n}^{m} e^{\mathrm{i} m \alpha \cdot \pi}-b_{n}^{-m} e^{-\mathrm{i} m \alpha \cdot \pi}\right) \frac{P_{n}^{m}(\cos \theta)}{\sin \theta}+\left.\sum_{m=-(n+1)}^{n+1} \frac{c_{n+1}^{m}(n+2)}{2 n+3} a_{n+1}^{m} \frac{\partial Y_{n+1}^{m}}{\partial \theta}\right|_{\phi=\phi_{0}}=0
\end{aligned}
$$

where $c_{n}^{m}$ are nonzero constants defined in (2.8) for $m=0,1, \ldots, n$. Furthermore, we have

$$
\begin{aligned}
& b_{n+1}^{m}+b_{n+1}^{-m}=0, \quad m=1, \ldots, n+1, \text { and } b_{n+1}^{0}=0, \\
& a_{n+1}^{m} e^{\mathrm{i} m \alpha \cdot \pi}+a_{n+1}^{-m} e^{-\mathbf{i} m \alpha \cdot \pi}=0, \quad m=1, \ldots, n+1, \text { and } a_{n+1}^{0}=0,
\end{aligned}
$$

where $c_{n+1}^{m}$ are nonzero constants defined in (2.8) for $m=0,1, \ldots, n+1$.

Proof. We first derive 4.1a), 4.1b and 4.1c). Since the generalized impedance condition (1.3) associated with $\boldsymbol{\eta}_{1}$ is imposed on $\Pi_{1}$ where $\boldsymbol{\eta}_{1} \equiv \infty$, using 2.24, we have

$$
\begin{aligned}
\mathbf{0}=\sum_{l=1}^{\infty} \sum_{m=-l}^{l} & -\frac{1}{\sqrt{l(l+1)}}\left\{\left.b_{l}^{m} l(l+1) p_{l}(k r) Y_{l}^{m}\right|_{\phi=0} \boldsymbol{e}_{\mathbf{1}}(\theta, 0)\right. \\
& \left.+\left(\left.a_{l}^{m} j_{l}(k r) \frac{m}{\sin \theta} Y_{l}^{m}\right|_{\phi=0}+\left.b_{l}^{m} \cdot q_{l}(k r) \frac{\partial Y_{l}^{m}}{\partial \theta}\right|_{\phi=0}\right) \boldsymbol{e}_{\mathbf{2}}(\theta, 0)\right\}
\end{aligned}
$$

where $\boldsymbol{e}_{1}(\theta, 0)$ and $\boldsymbol{e}_{2}(\theta, 0)$ are defined in 2.25$)$. From Remark 2.6, the lowest order of (4.7) with respect to the power of $r$ is $r^{0}$, which is contributed by $p_{1}(k r)$ and $q_{1}(k r)$. Similarly, the second lowest order of (4.7) with respect to the power of $r$ is $r^{1}$, which is contributed by $j_{1}(k r), p_{2}(k r)$ and $q_{2}(k r)$. Comparing the coefficient of $r^{0}$ and $r^{1}$ associated with $\boldsymbol{e}_{1}(\theta, 0)$ on both sides of $(4.7)$, utilizing the orthogonality property $(2.9)$, we can obtain (4.1a) and (4.1c). Substituting (4.1c) into 4.7), comparing the coefficient of $r^{1}$ in the resulting equation (4.7) associate with $\boldsymbol{e}_{2}(\theta, 0)$, using Lemma 2.4, we deduce that

$$
\left(a_{1}^{1} c_{1}^{1}-a_{1}^{-1} c_{1}^{-1}\right) P_{1}^{1}(\cos \theta)=0,
$$

where $c_{1}^{ \pm 1}$ are nonzero constants defined in $(2.8)$. In view of 4.8 , from $\sqrt{2.9}$ and $c_{1}^{1}=$ $c_{1}^{-1} \neq 0$, it yields that $4.1 \mathrm{~b}$. 
Since the generalized impedance condition $\sqrt{1.3}$ associated with $\boldsymbol{\eta}_{2}$ is imposed on $\widetilde{\Pi}_{2}$ where $\boldsymbol{\eta}_{2} \equiv 0$, by virtue of $(2.26)$ it yields that

$$
\begin{aligned}
\mathbf{0}=\mathbf{i} k & \sum_{l=1}^{\infty} \sum_{m=-l}^{l} \frac{1}{\sqrt{l(l+1)}}\left\{\left.a_{l}^{m} l(l+1) p_{l}(k r) Y_{l}^{m}\right|_{\phi=\phi_{0}} \cdot \boldsymbol{e}_{\mathbf{1}}\left(\theta, \phi_{0}\right)\right. \\
& \left.+\left(-\left.b_{l}^{m} j_{l}(k r) \cdot \frac{m}{\sin \theta} Y_{l}^{m}\right|_{\phi=\phi_{0}}+\left.a_{l}^{m} q_{l}(k r) \cdot \frac{\partial Y_{l}^{m}}{\partial \theta}\right|_{\phi=\phi_{0}}\right) \cdot \boldsymbol{e}_{\mathbf{2}}\left(\theta, \phi_{0}\right)\right\},
\end{aligned}
$$

where $\boldsymbol{e}_{1}\left(\theta, \phi_{0}\right)$ and $\boldsymbol{e}_{2}\left(\theta, \phi_{0}\right)$ are defined in 2.25$)$. From Remark 2.6, the lowest order of 4.9 with respect to the power of $r$ is $r^{0}$, which is contributed by $p_{1}(k r)$ and $q_{1}(k r)$. Similarly, the second lowest order of $(4.9)$ with respect to the power of $r$ is $r^{1}$, which is contributed by $j_{1}(k r), p_{2}(k r)$ and $q_{2}(k r)$. Comparing the coefficient of $r^{0}$ and $r^{1}$ associated with $\boldsymbol{e}_{1}\left(\theta, \phi_{0}\right)$ on both sides of $(4.9)$, utilizing the orthogonality property $(2.9)$, we can obtain (4.2a) and (4.2c).

Comparing the coefficient of $r^{1}$ in 4.9$)$ associate with $\boldsymbol{e}_{2}\left(\theta, \phi_{0}\right)$, using Lemma 2.4, we deduce that

$$
\left(b_{1}^{1} c_{1}^{1} e^{\mathbf{i} \alpha \cdot \pi}-b_{1}^{-1} c_{1}^{-1} e^{-\mathbf{i} \alpha \cdot \pi}\right) P_{1}^{1}(\cos \theta)=0,
$$

where $c_{1}^{ \pm 1}$ are nonzero constants defined in 2.8. In view of 4.10, from 2.9) and $c_{1}^{1}=$ $c_{1}^{-1} \neq 0$, it yields that $4.2 \mathrm{~b}$.

Now we are in the position to prove 4.4a, 4.5a and 4.6a under the assumption (4.3). Since the generalized impedance condition (1.3) associated with $\boldsymbol{\eta}_{1}$ is imposed on $\Pi_{1}$ where $\boldsymbol{\eta}_{1} \equiv \infty$, substituting 4.3 into 2.24 it yields that

$$
\begin{aligned}
\mathbf{0}=\sum_{l=n}^{\infty} \sum_{m=-l}^{l} & -\frac{1}{\sqrt{l(l+1)}}\left\{\left.b_{l}^{m} l(l+1) p_{l}(k r) Y_{l}^{m}\right|_{\phi=0} \boldsymbol{e}_{\mathbf{1}}(\theta, 0)\right. \\
& \left.+\left(\left.a_{l}^{m} j_{l}(k r) \frac{m}{\sin \theta} Y_{l}^{m}\right|_{\phi=0}+\left.b_{l}^{m} \cdot q_{l}(k r) \frac{\partial Y_{l}^{m}}{\partial \theta}\right|_{\phi=0}\right) \boldsymbol{e}_{\mathbf{2}}(\theta, 0)\right\},
\end{aligned}
$$

where $\boldsymbol{e}_{1}(\theta, 0)$ and $\boldsymbol{e}_{2}(\theta, 0)$ are defined in $(2.25)$.

The lowest order term in 4.11) with respect to the power of $r$ is $r^{n-1}$, which is contributed by $p_{n}(k r)$ and $q_{n}(k r)$ from Remark 2.6. Since $\boldsymbol{e}_{1}(\theta, \phi)$ and $\boldsymbol{e}_{2}(\theta, \phi)$ are linearly independent for any $\theta$ and $\phi$, where $\boldsymbol{e}_{i}(\theta, \phi)$ are defined in (2.9), from Lemma 2.4 comparing the coefficient of $r^{n-1}$ both sides of 4.11) associated with $\boldsymbol{e}_{1}(\theta, 0)$, we can obtain

$$
\sum_{m=0}^{n} c_{n}^{m}\left(b_{n}^{m}+b_{n}^{-m}\right) P_{n}^{m}(\cos \theta)=0 .
$$

Utilizing the orthogonality property $(2.9)$, since $c_{n}^{m} \neq 0$ for $\left.m \in[n]_{0}, 4.4 \mathrm{a}\right)$ holds.

From Remark 2.6 we know that the second lowest order term in in 4.11) with respect to the power of $r$ is $r^{n}$, which is related to $j_{n}(k r), p_{n+1}(k r)$ and $q_{n+1}(k r)$. Since $\boldsymbol{e}_{1}(\theta, \phi)$ and $\boldsymbol{e}_{2}(\theta, \phi)$ are linear independently for any $\theta$ and $\phi$, comparing the coefficient of $r^{n}$ both sides of (4.11) associated with $\boldsymbol{e}_{1}(\theta, 0)$, we can obtain

$$
\sum_{m=0}^{n+1} c_{n+1}^{m}\left(b_{n+1}^{m}+b_{n+1}^{-m}\right) P_{n+1}^{m}(\cos \theta)=0 .
$$

Using the orthogonality property $(2.9)$, together with the fact that $c_{n+1}^{m} \neq 0$ for $m \in$ $[n+1]_{0}$, we see that $4.6 \mathrm{a}$ holds.

Similarly, in view of Remark 2.6, comparing the coefficient of $r^{n}$ both sides of (4.11) associated with $\boldsymbol{e}_{2}(\theta, 0)$, we know that $4.5 \mathrm{a}$ hold. 
We proceed to derive 4.4b), 4.5b) and 4.6b) under the assumption (4.3). Since the generalized impedance condition (1.3) associated with $\boldsymbol{\eta}_{2}$ is imposed on $\widetilde{\Pi}_{2}$ where $\boldsymbol{\eta}_{2} \equiv 0$, substituting (4.3) into (2.26) it yields that

$$
\begin{aligned}
\mathbf{0}=\mathbf{i} k & \sum_{l=n}^{\infty} \sum_{m=-l}^{l} \frac{1}{\sqrt{l(l+1)}}\left\{\left.a_{l}^{m} l(l+1) p_{l}(k r) Y_{l}^{m}\right|_{\phi=\phi_{0}} \cdot \boldsymbol{e}_{\mathbf{1}}\left(\theta, \phi_{0}\right)\right. \\
& \left.+\left(-\left.b_{l}^{m} j_{l}(k r) \cdot \frac{m}{\sin \theta} Y_{l}^{m}\right|_{\phi=\phi_{0}}+\left.a_{l}^{m} q_{l}(k r) \cdot \frac{\partial Y_{l}^{m}}{\partial \theta}\right|_{\phi=\phi_{0}}\right) \cdot \boldsymbol{e}_{\mathbf{2}}\left(\theta, \phi_{0}\right)\right\} .
\end{aligned}
$$

where $\boldsymbol{e}_{1}\left(\theta, \phi_{0}\right)$ and $\boldsymbol{e}_{2}\left(\theta, \phi_{0}\right)$ are defined in (2.25).

The lowest order term in (4.12) with respect to the power of $r$ is $r^{n-1}$, which is contributed by $p_{n}(k r)$ and $q_{n}(k r)$ from Remark 2.6. Since $\boldsymbol{e}_{1}(\theta, \phi)$ and $\boldsymbol{e}_{2}(\theta, \phi)$ are linear independently for any $\theta$ and $\phi$, where $\boldsymbol{e}_{i}(\theta, \phi)$ are defined in 2.25), from Lemma 2.4. comparing the coefficient of $r^{n-1}$ both sides of 4.12 associated with $\boldsymbol{e}_{1}\left(\theta, \phi_{0}\right)$, we can obtain

$$
\sum_{m=0}^{n} c_{n}^{m}\left(a_{n}^{m} e^{\mathbf{i} m \alpha \cdot \pi}+a_{n}^{-m} e^{-\mathbf{i} m \alpha \cdot \pi}\right) P_{n}^{m}(\cos \theta)=0 .
$$

Using the orthogonality property (2.9), together with the fact that $c_{n}^{m} \neq 0$ for $m \in[n]_{0}$, we can obtain $4.4 \mathrm{~b}$.

From Remark 2.6 we know that the second lowest order term in 4.12 with respect to the power of $r$ is $r^{n}$, which is related to $j_{n}(k r), p_{n+1}(k r)$ and $q_{n+1}(k r)$. Since $\boldsymbol{e}_{1}(\theta, \phi)$ and $\boldsymbol{e}_{2}(\theta, \phi)$ are linearly independent for any $\theta$ and $\phi$, comparing the coefficient of $r^{n}$ both sides of 4.12) associated with $\boldsymbol{e}_{1}\left(\theta, \phi_{0}\right)$, we can get

$$
\sum_{m=0}^{n+1} c_{n+1}^{m}\left(a_{n+1}^{m} e^{\mathbf{i} m \alpha \cdot \pi}+a_{n+1}^{-m} e^{-\mathbf{i} m \alpha \cdot \pi}\right) P_{n+1}^{m}(\cos \theta)=0 .
$$

Utilizing the orthogonality property $(2.9)$, since $c_{n+1}^{m} \neq 0$ for $m \in[n+1]_{0}$, we derive (4.6b).

Similarly, in view of Remark 2.6, comparing the coefficient of $r^{n}$ both sides of (4.12) associated with $\boldsymbol{e}_{2}\left(\theta, \phi_{0}\right)$, we know that $4.5 \mathrm{~b}$ holds.

The proof is complete.

Theorem 4.2. Under the same setup in Lemma 4.1, we have that

$$
\operatorname{Vani}(\mathbf{E} ; \mathbf{0}) \geq N, \quad \text { if } \alpha \neq \frac{q}{2 p}, p=1, \ldots, N,
$$

where $N \in \mathbb{N}$ and for a fixed $p, q=1,2, \ldots, 4 p-1$.

Proof. We prove this theorem by induction. Assume that

$$
\alpha \neq \frac{1}{2} \text { and } \alpha \neq \frac{3}{2}
$$

we shall prove that $\operatorname{Vani}(\mathbf{E} ; \mathbf{0}) \geq 1$. Since the generalized impedance condition 1.3 associated with $\boldsymbol{\eta}_{1}$ is imposed on $\widetilde{\Pi}_{1}$ where $\boldsymbol{\eta}_{1} \equiv \infty$, from Lemma 4.1 we know that 4.1a) and (4.1b) hold. Similarly, since the generalized impedance condition $(1.3)$ associated with $\boldsymbol{\eta}_{2}$ is imposed on $\widetilde{\Pi}_{2}$ where $\boldsymbol{\eta}_{2} \equiv 0$, from Lemma 4.1 it yields that $4.2 \mathrm{a}$ and $4.2 \mathrm{~b}$.

Combing 4.1a), 4.1b), 4.2a) and (4.2b), it yields that




Under (4.14) we have

$$
\left|\begin{array}{cc}
1 & -1 \\
e^{\mathbf{i} \alpha \cdot \pi} & e^{-\mathbf{i} \alpha \cdot \pi}
\end{array}\right|=2 \cos (\alpha \cdot \pi) \neq 0,
$$

which implies that $a_{1}^{ \pm 1}=b_{1}^{ \pm 1}=0$ from 4.15. Since $a_{1}^{0}=b_{1}^{0}=0$, from Lemma 2.8, we prove $\operatorname{Vani}(\mathbf{E} ; \mathbf{0}) \geq 1$ under the assumption (4.14).

Assume that

$$
\alpha \neq \frac{1}{2}, \quad \alpha \neq \frac{1}{4}, \quad \alpha \neq \frac{3}{4}, \quad \alpha \neq \frac{5}{4}, \quad \alpha \neq \frac{3}{2} \text { and } \alpha \neq \frac{7}{4},
$$

which implies that $\operatorname{Vani}(\mathbf{E} ; \mathbf{0}) \geq 1$. Hence we have

$$
a_{1}^{ \pm 1}=b_{1}^{ \pm 1}=a_{1}^{0}=b_{1}^{0}=0
$$

from Lemma 2.8. Since the generalized impedance condition (1.3) associated with $\boldsymbol{\eta}_{1}$ is imposed on $\Pi_{1}$ where $\boldsymbol{\eta}_{1} \equiv \infty$, from Lemma 4.1 we have

$$
b_{2}^{0}=0, \quad b_{2}^{1}+b_{2}^{-1}=0, \quad b_{2}^{2}+b_{2}^{-2}=0
$$

by 4.4 a) and

$$
b_{3}^{m}+b_{3}^{-m}=0, \quad m=1,2,3, \text { and } b_{3}^{0}=0
$$

by 4.6a). Substituting 4.19 into the first equation of (4.5a), it yields that

$$
a_{2}^{1}-a_{2}^{-1}=0, \quad a_{2}^{2}-a_{2}^{-2}=0
$$

by noting $c_{3}^{m}=c_{3}^{-m} \neq 0$ for $m=1,2,3$, where $c_{3}^{m}$ and $c_{3}^{-m}$ are defined in $(2.8)$.

Similarly, in view of 4.17), using Lemma 4.1, we obtain that

$$
a_{2}^{0}=0, \quad a_{2}^{1} e^{\mathbf{i} \alpha \cdot \pi}+a_{2}^{-1} e^{-\mathbf{i} \alpha \cdot \pi}=0, \quad a_{2}^{2} e^{\mathbf{i} 2 \alpha \cdot \pi}+a_{2}^{-2} e^{-\mathbf{i} 2 \alpha \cdot \pi}=0
$$

by $4.4 \mathrm{~b}$ and

$$
a_{3}^{m} e^{\mathbf{i} m \alpha \pi}+a_{3}^{-m} e^{-\mathbf{i} m \alpha \pi}=0, \quad m=1,2,3, \text { and } a_{3}^{0}=0
$$

by $4.6 \mathrm{~b}$. Substituting 4.22 into the second equation of $4.5 \mathrm{~b}$, it yields that

$$
b_{2}^{1} e^{\mathbf{i} \alpha \cdot \pi}-b_{2}^{-1} e^{-\mathbf{i} \alpha \cdot \pi}=0, \quad b_{2}^{2} e^{\mathbf{i} 2 \alpha \cdot \pi}-b_{2}^{-2} e^{-\mathbf{i} 2 \alpha \cdot \pi}=0
$$

by using the fact that $c_{3}^{m}=c_{3}^{-m} \neq 0$ for $m=1,2$ and the definition of $Y_{3}^{m}(\theta, \phi)$, where $c_{3}^{m}$ and $c_{3}^{-m}$ are defined in 2.8). Combing (4.18), 4.20) and (4.21) with (4.23), we obtain that

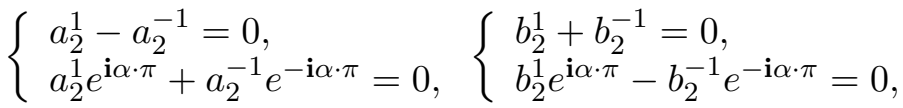

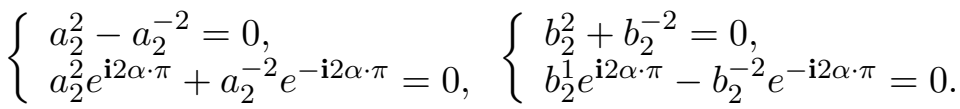

Under the assumption 4.16 it is easy to see that

$$
\left|\begin{array}{cc}
1 & -1 \\
e^{\mathbf{i} \alpha \cdot \pi} & e^{-\mathbf{i} \alpha \cdot \pi}
\end{array}\right|=2 \cos (\alpha \cdot \pi) \neq 0, \quad\left|\begin{array}{cc}
1 & -1 \\
e^{2 \mathbf{i} \alpha \cdot \pi} & e^{-2 \mathbf{i} \alpha \cdot \pi}
\end{array}\right|=2 \cos (2 \alpha \cdot \pi) \neq 0
$$

which imply that $a_{2}^{ \pm 1}=b_{2}^{ \pm 1}=a_{2}^{ \pm 2}=b_{2}^{ \pm 2}=0$ in view of (4.24). Due to (4.18) and (4.21), we have $a_{2}^{0}=b_{2}^{0}=0$, hence from Lemma 2.8 we prove $\operatorname{Vani}(\mathbf{E} ; \mathbf{0}) \geq 2$ under the assumption (4.16).

By the induction, we assume that

$$
\alpha \neq \frac{2 q+1}{2 p}, p=1, \ldots, n, \text { for a fixed } p, q=0,1, \ldots, 2 p-1 .
$$

Therefore, we know that $\operatorname{Vani}(\mathbf{E} ; \mathbf{0}) \geq n-1$ from the induction under the assumption (4.25), which implies that

$$
a_{l}^{m}=0 \text { for } l=1, \ldots, n-1 \text { and } m \in[l]_{0} .
$$


from Lemma 2.8.

Due to $(4.26)$ and the fact that the generalized impedance condition $(1.3)$ associated with $\boldsymbol{\eta}_{1}$ is imposed on $\widetilde{\Pi}_{1}$ where $\boldsymbol{\eta}_{1} \equiv \infty$, from Lemma 4.1, we have

$$
b_{n}^{m}+b_{n}^{-m}=0, \quad m=1, \ldots, n, \text { and } b_{n}^{0}=0
$$

by $4.4 a$ and

$$
b_{n+1}^{m}+b_{n+1}^{-m}=0, \quad m=1, \ldots, n+1, \text { and } b_{n+1}^{0}=0
$$

by 4.6a). Substituting 4.28 into the first equation of 4.5a), it yields that

$$
a_{2}^{m}-a_{2}^{-m}=0, \quad m=1, \ldots, n,
$$

by noting $c_{n+1}^{m}=c_{n+1}^{-m} \neq 0$ for $m=1, \ldots, n$, where $c_{n+1}^{m}$ and $c_{n+1}^{-m}$ are defined in (2.8).

Similarly, due to (4.26) and the fact that the generalized impedance condition (1.3) associated with $\boldsymbol{\eta}_{2}$ is imposed on $\widetilde{\Pi}_{2}$ where $\boldsymbol{\eta}_{2} \equiv 0$, using Lemma 4.1 , we get that

$$
a_{n}^{m} e^{\mathbf{i} m \alpha \pi}+a_{n}^{-m} e^{-\mathbf{i} m \alpha \pi}=0, \quad m=1, \ldots, n, \text { and } a_{n}^{0}=0
$$

by $4.4 \mathrm{~b}$ ) and

$$
a_{n+1}^{m} e^{\mathbf{i} m \alpha \pi}+a_{n+1}^{-m} e^{-\mathbf{i} m \alpha \pi}=0, \quad m=1, \ldots, n+1, \text { and } a_{n+1}^{0}=0
$$

by (4.6b). Substituting (4.31) into the second equation of 4.5b), it yields that

$$
b_{n}^{m} e^{\mathbf{i} m \alpha \cdot \pi}-b_{n}^{-m} e^{-\mathbf{i} m \alpha \cdot \pi}=0, \quad m=1, \ldots, n
$$

by using the fact that $c_{n+1}^{m}=c_{n+1}^{-m} \neq 0$ for $m=1, \ldots, n$ and the definition of $Y_{n+1}^{m}(\theta, \phi)$, where $c_{n+1}^{m}$ and $c_{n+1}^{-m}$ are defined in 2.8 .

Combing 4.27, 4.29) and 4.30 with 4.32, we obtain that

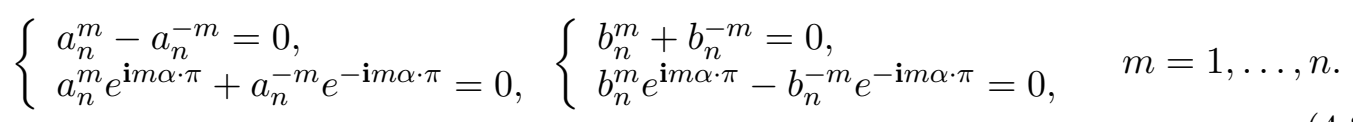

Under the assumption 4.25 it is not difficult to see that

$$
\left|\begin{array}{cc}
1 & -1 \\
e^{\mathbf{i} m \alpha \cdot \pi} & e^{-\mathbf{i} m \alpha \cdot \pi}
\end{array}\right|=2 \cos (m \alpha \cdot \pi) \neq 0,
$$

which imply that $a_{n}^{ \pm m}=b_{n}^{ \pm m}=0$ in view of (4.33). Due to (4.27) and (4.30), we have $a_{n}^{0}=b_{n}^{0}=0$, hence from Lemma 2.8 we prove $\operatorname{Vani}(\mathbf{E} ; \mathbf{0}) \geq n$ under the assumption (4.16).

The proof is complete.

In the following two theorems, we consider the generalized impedance edge-corner $\mathcal{E}\left(\widetilde{\Pi}_{1}, \widetilde{\Pi}_{2}, \boldsymbol{l}\right)$ where the generalize impedance parameter $\boldsymbol{\eta}_{2}$ on $\widetilde{\Pi}_{2}$ satisfies (iii) in 1.2 and has the expansion $(3.1 \mathrm{~b})$, whereas the generalize impedance parameter $\boldsymbol{\eta}_{1}$ on $\Pi_{1}$ satisfies either (i) or (ii) in (1.2). In the sequel, we shall make use of the reflection principles for the Maxwell equations from 13, 14.

For any two-dimensional plane $\Pi \in \mathbb{R}^{3}$, let $\nu_{\Pi}$ and $\mathcal{R}_{\Pi}$ be respectively the unit normal to $\Pi$ and the reflection with respect to $\Pi$ in $\mathbb{R}^{3}$.

Lemma 4.3. [14, Theorems 2.1 and 2.2] Consider a generalized impedance edge-corner $\mathcal{E}\left(\widetilde{\Pi}_{1}, \widetilde{\Pi}_{2}, \boldsymbol{l}\right) \Subset \Omega$ with $\angle\left(\Pi_{1}, \Pi_{2}\right)=\phi_{0}=\alpha \pi$, where $\alpha \in(0,1)$. Assume that the generalize impedance parameter $\boldsymbol{\eta}_{2}$ on $\widetilde{\Pi}_{2}$ satisfies (iii) in 1.2 and has the expansion $3.1 \mathrm{~b}$ while the generalize impedance parameter $\boldsymbol{\eta}_{1}$ on $\widetilde{\Pi}_{1}$ satisfies (ii) in 1.2 (i.e., $\boldsymbol{\eta}_{1} \equiv \infty$ ). Recall that $\Pi_{1}$ be a plane containing $\widetilde{\Pi}_{1}$. Let $\widetilde{\Pi}_{2}^{\prime}=\mathcal{R}_{\Pi_{1}}\left(\widetilde{\Pi}_{2}\right)$. Then

$$
\nu_{\widetilde{\Pi}_{2}^{\prime}} \wedge(\nabla \wedge \mathbf{E})+\widetilde{\boldsymbol{\eta}}_{2}\left(\nu_{\widetilde{\Pi}_{2}^{\prime}} \wedge \mathbf{E}\right) \wedge \nu_{\widetilde{\Pi}_{2}^{\prime}}=\mathbf{0} \text { on } \widetilde{\Pi}_{2}^{\prime},
$$


where $\nu_{\Pi_{2}^{\prime}}$ is the unit normal to $\Pi_{2}^{\prime}$ directed to the interior of $\mathcal{E}\left(\widetilde{\Pi}_{1}, \widetilde{\Pi}_{2}^{\prime}, \boldsymbol{l}\right)$ and $\widetilde{\boldsymbol{\eta}}_{2}(\mathbf{x})=$ $\boldsymbol{\eta}_{2}\left(\mathcal{R}_{\Pi_{1}}(\mathbf{x})\right)$ for $\mathbf{x} \in \widetilde{\Pi}_{2}^{\prime}$.

Similarly, consider a generalized impedance edge-corner $\mathcal{E}\left(\widetilde{\Pi}_{1}, \widetilde{\Pi}_{2}, \boldsymbol{l}\right) \Subset \Omega$ with $\angle\left(\Pi_{1}, \Pi_{2}\right)=$ $\phi_{0}=\alpha \pi$, where $\alpha \in(0,1)$. Assume that the generalize impedance parameter $\boldsymbol{\eta}_{2}$ on $\widetilde{\Pi}_{2}$ satisfies (iii) in (1.2) and has the expansion (3.1b) while the generalize impedance parameter $\boldsymbol{\eta}_{1}$ on $\widetilde{\Pi}_{1}$ satisfies (i) in 1.2 (i.e., $\boldsymbol{\eta}_{1} \equiv 0$ ). Recall that $\Pi_{1}$ be a plane containing $\widetilde{\Pi}_{1}$. Let $\widetilde{\Pi}_{2}^{\prime}=\mathcal{R}_{\Pi_{1}}\left(\widetilde{\Pi}_{2}\right)$. Then

$$
\nu_{\widetilde{\Pi}_{2}^{\prime}} \wedge(\nabla \wedge \mathbf{E})+\widetilde{\boldsymbol{\eta}}_{2}\left(\nu_{\widetilde{\Pi}_{2}^{\prime}} \wedge \mathbf{E}\right) \wedge \nu_{\widetilde{\Pi}_{2}^{\prime}}=\mathbf{0} \text { on } \widetilde{\Pi}_{2}^{\prime},
$$

where $\nu_{\Pi_{2}^{\prime}}$ is the unit normal to $\Pi_{2}^{\prime}$ directed to the interior of $\mathcal{E}\left(\widetilde{\Pi}_{1}, \widetilde{\Pi}_{2}^{\prime}, \boldsymbol{l}\right)$ and $\widetilde{\boldsymbol{\eta}}_{2}(\mathbf{x})=$ $\boldsymbol{\eta}_{2}\left(\mathcal{R}_{\Pi_{1}}(\mathbf{x})\right)$ for $\mathbf{x} \in \widetilde{\Pi}_{2}^{\prime}$.

Theorem 4.4. Let $\mathbf{E}$ be a solution to (1.1). Consider a generalized impedance edgecorner $\mathcal{E}\left(\widetilde{\Pi}_{1}, \widetilde{\Pi}_{2}, \boldsymbol{l}\right) \Subset \Omega$ with $\angle\left(\Pi_{1}, \Pi_{2}\right)=\phi_{0}=\alpha \pi$, where $\alpha \in(0,2)$ and $\alpha \neq 1$. Assume that the generalize impedance parameter $\boldsymbol{\eta}_{2}$ on $\widetilde{\Pi}_{2}$ satisfies (iii) in 1.2 and has the expansion (3.1b) while the generalize impedance parameter $\boldsymbol{\eta}_{1}$ on $\widetilde{\Pi}_{1}$ satisfies (ii) in (1.2) (i.e., $\left.\boldsymbol{\eta}_{1} \equiv \infty\right)$. Then

$$
\operatorname{Vani}(\mathbf{E} ; \mathbf{0}) \geq N, \quad \text { if } \alpha \neq \frac{q}{2 p}, p=1, \ldots, N,
$$

where $N \in \mathbb{N}$ and for a fixed $p, q=1,2, \ldots, 4 p-1$.

Proof. Let $\widetilde{\Pi}_{2}^{\prime}=\mathcal{R}_{\Pi_{1}}\left(\widetilde{\Pi}_{2}\right)$, where $\Pi_{1}$ is a plane containing $\Pi_{1}$. With the help of Lemma 4.3 , we know that $\mathbf{E}$ satisfies the generalized impedance boundary condition (4.34) on $\widetilde{\Pi}_{2}^{\prime}$. Since $\mathbf{x} \in \widetilde{\Pi}_{2}$, we have the spherical coordinate of $\mathbf{x}=\left(r, \theta, \phi_{0}\right)$, where $0 \leq r \leq h$, $\theta \in[-\pi, \pi]$ and $\phi_{0}=\alpha \pi$. It is clear that the spherical coordinate of $\left.\mathcal{R}_{\Pi_{1}}(\mathbf{x})\right)$, where $\mathbf{x} \in \widetilde{\Pi}_{2}$, is given by

$$
\left(r, \theta, \phi_{1}\right) \text {, where } \phi_{1}=2-\alpha \in(0,2) .
$$

Recall that $\boldsymbol{\eta}_{2}$ has the expansion (3.1b). Although $\mathbf{x} \in \widetilde{\Pi}_{2}$ and $\left.\mathcal{R}_{\Pi_{1}}(\mathbf{x})\right) \in \widetilde{\Pi}_{2}^{\prime}$ have different azimuthal angles but they have the same polar angle $\theta$, hence from Definition 2.2 , we know that $\widetilde{\boldsymbol{\eta}}_{2}$ has the same expansion $(3.1 \mathrm{~b})$ as $\boldsymbol{\eta}_{2}$.

Furthermore, the dihedral angle between $\widetilde{\Pi}_{2}$ and $\widetilde{\Pi}_{2}^{\prime}$ satisfies

$$
\angle\left(\widetilde{\Pi}_{2}, \widetilde{\Pi}_{2}^{\prime}\right)= \begin{cases}2 \alpha \pi \in(0, \pi], & \alpha \in(0,1 / 2), \\ 2(1-\alpha) \pi \in(0, \pi], & \alpha \in[1 / 2,1), \\ 2(\alpha-1) \pi \in(0, \pi], & \alpha \in(1,3 / 2), \\ 2(2-\alpha) \pi \in(0, \pi], & \alpha \in[3 / 2,2),\end{cases}
$$

We divide our remaining proof into four separate cases. Recall that that the Maxwell system (1.1) is invariant under rigid motions. Without loss of generality, we assume that the generalized impedance edge-corner $\mathcal{E}\left(\widetilde{\Pi}_{2}, \widetilde{\Pi}_{2}^{\prime}, \boldsymbol{l}\right) \Subset \Omega$ are placed as shown in Figure 1 .

Case 1. If $\alpha \in(0,1 / 2)$, then $2 \alpha \in(0,1)$. By virtue of Theorem 3.6, if

$$
2 \alpha \neq \frac{q}{p}, \quad p=1, \ldots, N, \text { for a fixed } p, q=1, \ldots, p-1,
$$

we have $\operatorname{Vani}(\mathbf{E} ; \mathbf{0}) \geq N$. It is easy to see that 4.36$)$ is equivalent to

$$
\alpha \neq \frac{q}{2 p}, \quad p=1, \ldots, N, \text { for a fixed } p, q=1, \ldots, p-1 .
$$


Case 2. If $\alpha \in[1 / 2,1)$, then $2(1-\alpha) \in(0,1]$. By virtue of Theorem 3.6, if

$$
2(1-\alpha) \neq \frac{q}{p}, \quad p=1, \ldots, N, \text { for a fixed } p, q=1, \ldots, p,
$$

we have $\operatorname{Vani}(\mathbf{E} ; \mathbf{0}) \geq N$. It is easy to see that 4.38 is equivalent to

$$
\alpha \neq \frac{q}{2 p}, \quad p=1, \ldots, N \text {, for a fixed } p, q=p, \ldots, 2 p-1 .
$$

Case 3. If $\alpha \in(1,3 / 2)$, then $2(\alpha-1) \in(0,1)$. By virtue of Theorem 3.6, if

$$
2(\alpha-1) \neq \frac{q}{p}, \quad p=1, \ldots, N, \text { for a fixed } p, q=1, \ldots, p-1,
$$

we have $\operatorname{Vani}(\mathbf{E} ; \mathbf{0}) \geq N$. It is easy to see that 4.40 is equivalent to

$$
\alpha \neq \frac{q}{2 p}, \quad p=1, \ldots, N, \text { for a fixed } p, q=2 p+1, \ldots, 3 p-1 .
$$

Case 4. If $\alpha \in[3 / 2,2)$, then $2(2-\alpha) \in(0,1]$. By virtue of Theorem 3.6, if

$$
2(2-\alpha) \neq \frac{q}{p}, \quad p=1, \ldots, N, \text { for a fixed } p, q=1, \ldots, p-1,
$$

we have $\operatorname{Vani}(\mathbf{E} ; \mathbf{0}) \geq N$. It is easy to see that 4.42 is equivalent to

$$
\alpha \neq \frac{q}{2 p}, \quad p=1, \ldots, N, \text { for a fixed } p, q=3 p, \ldots, 4 p-1 .
$$

In view of (4.37), (4.39), 4.41) and (4.43), we finish the proof of this theorem.

With the help of Lemma 4.3, using the similar argument for proving Theorem 4.4, we can prove the following theorem, where the detailed proof is omitted.

Theorem 4.5. Let $\mathbf{E}$ be a solution to (1.1). Consider a generalized impedance edgecorner $\mathcal{E}\left(\widetilde{\Pi}_{1}, \widetilde{\Pi}_{2}, \boldsymbol{l}\right) \Subset \Omega$ with $\angle\left(\Pi_{1}, \Pi_{2}\right)=\phi_{0}=\alpha \pi$, where $\alpha \in(0,1)$. Assume that the generalize impedance parameter $\boldsymbol{\eta}_{2}$ on $\widetilde{\Pi}_{2}$ satisfies (iii) in 1.2 and has the expansion (3.1b) while the generalize impedance parameter $\boldsymbol{\eta}_{1}$ on $\widetilde{\Pi}_{1}$ satisfies (i) in 1.2 (i.e., $\left.\boldsymbol{\eta}_{1} \equiv 0\right)$. Then

$$
\operatorname{Vani}(\mathbf{E} ; \mathbf{0}) \geq N, \quad \text { if } \alpha \neq \frac{q}{2 p}, p=1, \ldots, N,
$$

where $N \in \mathbb{N}$ and for a fixed $p, q=1,2, \ldots, 4 p-1$.

\section{IRRATIONAL INTERSECTIONS AND INFINITE VANISHING ORDERS}

From the results derived in Sections 3 to 4 , one can identify that the vanishing order of the eigenfunction $\mathbf{E}$ at a generalized impedance edge-corner relies on the degree of the dihedral angle of the underlying corner. Next, we introduce the irrational and rational edge-corners, and then, based on the results in Sections 3 to 4 , we show that the vanishing order of the eigenfunction at an irrational edge-corner is generically infinity and hence it vanishes identically in $\Omega$, namely strong uniqueness continuation principle holds in such a case.

Definition 5.1. Let $\mathcal{E}\left(\widetilde{\Pi}_{1}, \widetilde{\Pi}_{2}, \boldsymbol{l}\right)$ be an edge-corner defined in Section 1 and the corresponding dihedral angle of $\widetilde{\Pi}_{1}$ and $\widetilde{\Pi}_{2}$ is denoted by $\phi_{0}=\alpha \pi, \alpha \in(0,2)$ and $\alpha \neq 1$. If $\alpha$ is an irrational number, then the edge-corner is called irrational. If $\alpha$ is a rational number of the form $q / p$ with $p, q \in \mathbb{N}$ being irreducible, the edge-corner is called rational and $p$ is referred to as its rational degree.

We readily have the following theorem from Theorems $3.6,4.2,4.4$ and 4.5 . 
Theorem 5.2. Let $\mathbf{E}$ be a solution to (1.1). Consider an irrational generalized impedance edge-corner $\mathcal{E}\left(\widetilde{\Pi}_{1}, \widetilde{\Pi}_{2}, \boldsymbol{l}\right) \Subset \Omega$ with $\angle\left(\Pi_{1}, \Pi_{2}\right)=\phi_{0}=\alpha \pi$, where $\alpha \in(0,2)$ and $\alpha \neq 1$. Under the same requirement on $\boldsymbol{\eta}_{j}, j=1,2$, to either one from Theorems 3.6. 4.2. 4.4 and 4.5 , it holds that

$$
\operatorname{Vani}(\mathbf{E} ; \mathbf{0})=+\infty, \quad \mathbf{0} \in \boldsymbol{l} .
$$

\section{Applications to inverse electromagnetic SCattering problems}

In this section, we consider two applications of the UCP results established in the previous sections to the inverse electromagnetic scattering problems. In what follows, we first present the mathematical formulation of the inverse problem of determining an impenetrable obstacle from its associated electromagnetic far-field measurement. It is a prototypical model problem for many real applications including radar/sonar, nondestructive testing and medical imaging.

6.1. Unique identifiability results for inverse obstacle scattering problems. Let $\Omega \subset \mathbb{R}^{3}$ be a bounded Lipschitz domain such that $\mathbb{R}^{3} \backslash \bar{\Omega}$ is connected, and the incident electric and magnetic fields be of the form

$$
\mathbf{E}^{i}(\mathbf{x}):=\mathbf{p} e^{\mathbf{i} k \mathbf{x} \cdot \mathbf{d}}, \quad \mathbf{H}^{i}(\mathbf{x}) \quad:=\frac{1}{\mathbf{i} k} \nabla \wedge \mathbf{p} e^{\mathbf{i} k \mathbf{x} \cdot \mathbf{d}}=\mathbf{d} \wedge \mathbf{p} e^{\mathbf{i} k \mathbf{x} \cdot \mathbf{d}},
$$

which are known as the time-harmonic electromagnetic plane waves, with $\mathbf{p} \in \mathbb{R}^{3} \backslash\{\mathbf{0}\}, k \in$ $\mathbb{R}_{+}$and $\mathbf{d} \in \mathbb{S}^{2}:=\left\{\mathbf{x} \in \mathbb{R}^{3} ;|\mathbf{x}|=1\right\}$ representing respectively the polarization, wave number and direction of propagation, and it holds that $\mathbf{p} \perp \mathbf{d}$. The associated forward scattering problem can be described by the following the time-harmonic Maxwell equations (cf. [6]):

$$
\left\{\begin{array}{l}
\nabla \wedge \mathbf{E}-\mathbf{i} k \mathbf{H}=0 \quad \text { in } \quad \mathbb{R}^{3} \backslash \bar{\Omega}, \\
\nabla \wedge \mathbf{H}+\mathbf{i} k \mathbf{E}=0 \quad \text { in } \quad \mathbb{R}^{3} \backslash \bar{\Omega}, \\
\mathbf{E}(\mathbf{x})=\mathbf{E}^{i}(\mathbf{x})+\mathbf{E}^{s}(\mathbf{x}), \\
\mathbf{H}(\mathbf{x})=\mathbf{H}^{i}(\mathbf{x})+\mathbf{H}^{s}(\mathbf{x}), \\
\mathscr{B}(\mathbf{E})=\mathbf{0} \quad \text { on } \partial \Omega, \\
\lim _{|\mathbf{x}| \rightarrow \infty}\left(\mathbf{H}^{s} \wedge \mathbf{x}-|\mathbf{x}| \mathbf{E}^{s}\right)=\mathbf{0},
\end{array}\right.
$$

where $\mathbf{E}=\left(E_{1}, E_{2}, E_{3}\right)$ and $\mathbf{H}=\left(H_{1}, H_{2}, H_{3}\right)$ are respectively the total electric and magnetic fields formed by the incident fields $\mathbf{E}^{i}(\mathbf{x})$ and $\mathbf{H}^{i}(\mathbf{x})$ and scattered fields $\mathbf{E}^{s}(\mathbf{x})$ and $\mathbf{H}^{s}(\mathbf{x})$. The last equation of 6.2 is the Silver-Müller radiation condition. The boundary condition $\mathscr{B}(\mathbf{E})$ on $\partial \Omega$ could be either of the following three conditions:

(1) the Dirichlet condition (corresponding to that $\Omega$ is a perfectly electric conducting (PEC) obstacle):

$$
\mathscr{B}(\mathbf{E})=\nu \wedge \mathbf{E}
$$

(2) the Neumann condition (corresponding to that $\Omega$ is a perfectly magnetic conducting (PMC) obstacle):

$$
\mathscr{B}(\mathbf{E})=\nu \wedge(\nabla \wedge \mathbf{E})
$$

(3) the impedance condition (corresponding to that $\Omega$ is an impedance obstacle):

$$
\mathscr{B}(\mathbf{E})=\nu \wedge(\nabla \wedge \mathbf{E})+\boldsymbol{\eta}(\nu \wedge \mathbf{E}) \wedge \nu, \Re(\boldsymbol{\eta}) \geq 0 \text { and } \Im(\boldsymbol{\eta})<0,
$$

where $\nu$ denotes the exterior unit normal vector to $\partial \Omega$ and $\boldsymbol{\eta} \in L^{\infty}(\Omega)$. We would also like to point out that the conditions $\Re(\boldsymbol{\eta}) \geq 0$ and $\Im(\boldsymbol{\eta})<0$ are the physical requirement.

In what follows, in order to ease the exposition and similar to our notation in 1.2 (1.2), we unify the three types of boundary conditions as

$$
\mathscr{B}(\mathbf{E})=\nu \wedge(\nabla \wedge \mathbf{E})+\boldsymbol{\eta}(\nu \wedge \mathbf{E}) \wedge \nu \quad \text { on } \partial \Omega,
$$


where the cases that $\boldsymbol{\eta}=\infty$ and $\boldsymbol{\eta}=0$ stand for the Dirichlet and Neumann boundary conditions respectively.

For the forward scattering problem $(6.2)$, it is known that there exists a unique pair of solutions $(\mathbf{E}, \mathbf{H}) \in H_{\text {loc }}\left(\operatorname{curl}, \mathbb{R}^{3} \backslash \bar{\Omega}\right) \times H_{\text {loc }}\left(\operatorname{curl}, \mathbb{R}^{3} \backslash \bar{\Omega}\right)$ (cf. [17]). Furthermore, the radiating fields $\mathbf{E}^{s}$ and $\mathbf{H}^{s}$ to 6.2 possess the following asymptotic expansions

$$
\begin{aligned}
& \mathbf{E}^{s}(\mathbf{x} ; \Omega, k, \mathbf{d}, \mathbf{p})=\frac{e^{\mathbf{i} k \mathbf{x} \cdot \mathbf{d}}}{|\mathbf{x}|}\left\{\mathbf{E}_{\infty}(\hat{\mathbf{x}} ; \Omega, k, \mathbf{d}, \mathbf{p})+\mathcal{O}\left(\frac{1}{|\mathbf{x}|}\right)\right\} \quad \text { as } \quad|\mathbf{x}| \rightarrow \infty \\
& \mathbf{H}^{s}(\mathbf{x} ; \Omega, k, \mathbf{d}, \mathbf{p})=\frac{e^{\mathbf{i} k \mathbf{x} \cdot \mathbf{d}}}{|\mathbf{x}|}\left\{\mathbf{H}_{\infty}(\hat{\mathbf{x}} ; \Omega, k, \mathbf{d}, \mathbf{p})+\mathcal{O}\left(\frac{1}{|\mathbf{x}|}\right)\right\} \quad \text { as } \quad|\mathbf{x}| \rightarrow \infty
\end{aligned}
$$

which hold uniformly in the angular variable $\hat{\mathbf{x}}=\mathbf{x} /|\mathbf{x}| \in \mathbb{S}^{2}$. The functions $\mathbf{E}_{\infty}(\hat{\mathbf{x}})$ and $\mathbf{H}_{\infty}(\hat{\mathbf{x}})$ in (6.7) are called, respectively, the electric and magnetic far field patterns, and both are analytic on the entire unit sphere $\mathbb{S}^{2}$. As above and also in what follows, the notation $\mathbf{U}(\mathbf{x} ; \Omega, \mathbf{p}, k, \mathbf{d})$ will be frequently used to specify the dependence of a given function $\mathbf{U}$ on the scatterer $\Omega$, the polarization $\mathbf{p}$, the wave number $k$ and the incident direction $\mathbf{d}$.

The inverse electromagnetic obstacle scattering problem corresponding to $(6.2)$ is to recover $\Omega$ (and $\boldsymbol{\eta}$ as well in the impedance case) by the knowledge of the far-field pattern $\mathbf{E}_{\infty}(\hat{\mathbf{x}} ; \Omega, \mathbf{p}, k, \mathbf{d})$ (or equivalently $\mathbf{H}_{\infty}(\hat{\mathbf{x}} ; \Omega, \mathbf{p}, k, \mathbf{d})$ ). By introducing an operator $\mathcal{F}$ which sends the obstacle to the corresponding far-field pattern, defined by the forward scattering system (6.2), the aforementioned inverse problem can be formulated as

$$
\mathcal{F}(\Omega, \boldsymbol{\eta})=\mathbf{E}_{\infty}(\hat{\mathbf{x}} ; \Omega, k, \mathbf{d}, \mathbf{p}) .
$$

It can be directly verified that the inverse problem $(6.8)$ is nonlinear and moreover it is ill-conditioned (cf. [6]). It is a longstanding problem that one can establish the one-toone correspondence for (6.8) by a single far-field pattern or a finite number of far-field patterns (namely with a fixed triplet of $k, \mathbf{d}$ and $\mathbf{p}$ or a finite number of triplets of $k, \mathbf{d}$ and $\mathbf{p}$ ); see the recent survey paper [7] by Colton and Kress for more discussions about the historical developments of this fundamental problem.

Under the assumption that $\Omega$ is a polyhedral obstacle associated with $\boldsymbol{\eta} \equiv 0$ or $\boldsymbol{\eta} \equiv \infty$, the unique correspondence, a.k.a unique identifiability, for the inverse problem 6.8 by a single far-field measurement was established in the literature; see [11 14. However, it is still unclear whether one can establish the unique identifiability for an impedance obstacle of the polyhedral shape, even for the case that $\boldsymbol{\eta}$ is a nonzero constant, and a fortiori in our present paper $\boldsymbol{\eta}$ is a generalised impedance parameter which can be $0, \infty$ or a variable function. To be more specific about the generalised impedance obstacle, we introduce the following definition.

Definition 6.1. Let $\Omega$ be an open and bounded polyhedron in $\mathbb{R}^{3}$. Hence, $\partial \Omega$ possesses finitely many edge-corners that are formed by the intersections of any two adjacent faces of $\partial \Omega . \Omega$ is said to be irrational if all of its edge-corners are irrational, otherwise it is called rational, and the smallest degree among the rational degrees of all of its rational corners is referred to the degree of the polyhedron.

Definition 6.2. $(\Omega, \boldsymbol{\eta})$ is said to be an admissible polyhedral obstacle if $\Omega$ is an open bounded polyhedron and $\boldsymbol{\eta}$ fulfils the following requirements.

(1) For each face of $\partial \Omega$, say $\widetilde{\Pi}$, and each edge of $\widetilde{\Pi}$, say $\boldsymbol{l}$, there exists a neighbourhood $\Sigma_{\boldsymbol{l}}:=B_{\rho}(\boldsymbol{l}) \cap \widetilde{\Pi}$ with $\rho \in \mathbb{R}_{+}$and $B_{\rho}(\boldsymbol{l}):=\left\{\mathbf{x} \in \mathbb{R}^{3} ;\left|\mathbf{x}-\mathbf{x}^{\prime}\right|<\rho, \forall \mathbf{x}^{\prime} \in \boldsymbol{l}\right\}$, such that either $\left.\boldsymbol{\eta}\right|_{\Sigma_{l}}=0$, or $\left.\boldsymbol{\eta}\right|_{\Sigma_{l}}=\infty$, or $\left.\eta\right|_{\Sigma_{l}} \in \mathcal{A}(\boldsymbol{l})$.

(2) On any open subset of the other part of $\partial \Omega$ other than the neighbourhood of each edge of $\partial \Omega$ introduced in (1), $\boldsymbol{\eta}$ can be 0 , or $\infty$ or $\eta \in L^{\infty}$.

(3) In the case $\boldsymbol{\eta} \in L^{\infty}$, one has that $\Re(\boldsymbol{\eta}) \geq 0$ and $\Im(\boldsymbol{\eta})<0$. 
Definition 6.3. $\Omega$ is said to be an admissible complex polyhedral obstacle if it consists of finitely many admissible polyhedral obstacles. That is,

$$
(\Omega, \boldsymbol{\eta})=\bigcup_{j=1}^{l}\left(\Omega_{j}, \boldsymbol{\eta}_{j}\right),
$$

where $l \in \mathbb{N}$ and each $\left(\Omega_{j}, \boldsymbol{\eta}_{j}\right)$ is an admissible polyhedral obstacle. Here, we define

$$
\boldsymbol{\eta}=\sum_{j=1}^{l} \boldsymbol{\eta}_{j} \chi_{\partial \Omega_{j}} .
$$

Moreover, $\Omega$ is said to be irrational if all of its component polyhedral obstacles are irrational, otherwise it is said to be rational. For the latter case, the smallest degree among all the degrees of its rational components is defined to be the degree of the complex obstacle $\Omega$.

Next, we first derive a local unique identifiability result in determining an admissible complex irrational polyhedral obstacle by a single far-field pattern.

Theorem 6.4. Consider a fixed triplet of $k \in \mathbb{R}_{+}, \mathbf{d} \in \mathbb{S}^{2}$ and $\mathbf{p} \in \mathbb{R}^{3} \backslash\{\mathbf{0}\}$. Let $(\Omega, \eta)$ and $(\widetilde{\Omega}, \widetilde{\eta})$ be two admissible complex irrational obstacles, with $\mathbf{E}_{\infty}$ and $\widetilde{\mathbf{E}}_{\infty}$ being their corresponding far-field patterns and $\mathbf{G}$ being the unbounded connected component of $\mathbb{R}^{3} \backslash \overline{(\Omega \cup \widetilde{\Omega})}$. If $\mathbf{E}_{\infty}$ and $\widetilde{\mathbf{E}}_{\infty}$ are the same in the sense that

$$
\mathbf{E}_{\infty}(\hat{\mathbf{x}} ; \Omega, k, \mathbf{d}, \mathbf{p})=\widetilde{\mathbf{E}}_{\infty}(\hat{\mathbf{x}} ; \widetilde{\Omega}, k, \mathbf{d}, \mathbf{p}), \text { for all } \hat{\mathbf{x}} \in \mathbb{S}^{2},
$$

then $(\partial \Omega \backslash \partial \overline{\widetilde{\Omega}}) \bigcup(\partial \widetilde{\Omega} \backslash \partial \bar{\Omega})$ cannot possess an edge-corner on $\partial \mathbf{G}$. Moreover,

$$
\boldsymbol{\eta}=\widetilde{\boldsymbol{\eta}} \quad \text { on } \quad \partial \Omega \cap \partial \widetilde{\Omega} \cap \partial \mathbf{G} .
$$

Proof. We prove the theorem by contradiction. Assume that $(\partial \Omega \backslash \partial \widetilde{\widetilde{\Omega}}) \bigcup(\partial \widetilde{\Omega} \backslash \partial \bar{\Omega})$ has an edge corner $\mathbf{x}_{c}$ on $\partial \mathbf{G}$. Then, $\mathbf{x}_{c}$ is either located at $\partial \Omega$ or $\partial \widetilde{\Omega}$. Without loss of generality, we assume that $\mathbf{x}_{c}$ is an edge corner of $\partial \widetilde{\Omega}$, which also indicates that $\mathbf{x}_{c}$ lies outside $\Omega$. Let $h \in \mathbb{R}_{+}$be sufficiently small such that $B_{h}\left(\mathbf{x}_{c}\right) \Subset \mathbb{R}^{2} \backslash \bar{\Omega}$, then we have

$$
B_{h}\left(\mathbf{x}_{c}\right) \cap \partial \widetilde{\Omega}=\widetilde{\Pi}_{\ell}, \quad \ell=1,2,
$$

where $\widetilde{\Pi}_{\ell}$ are two flat subsets lying on the faces of $\widetilde{\Omega}$ that intersect at $\mathbf{x}_{c}$. Moreover, for the subsequent use, we let $h$ be smaller than $\rho$, where $\rho$ is the parameter in Definition 6.2. Hence we have an edge-corner $\mathcal{E}\left(\widetilde{\Pi}_{1}, \widetilde{\Pi}_{2}, \boldsymbol{l}\right) \in \partial \mathbf{G}$ with $\mathbf{x}_{c} \in \boldsymbol{l}$, where $\mathbf{G}$ is the unbounded connected component of $\mathbb{R}^{3} \backslash \overline{(\Omega \cup \widetilde{\Omega})}$. By (6.9) and the Rellich theorem (cf. [6]), we know that

$$
\mathbf{E}(\mathbf{x} ; k, \mathbf{d}, \mathbf{p})=\widetilde{\mathbf{E}}(\mathbf{x} ; k, \mathbf{d}, \mathbf{p}), \quad \mathbf{x} \in \mathbf{G} .
$$

Since $\widetilde{\Pi}_{\ell} \subset \partial \mathbf{G}, \ell=1,2$, combining 6.11 with the generalized boundary condition 6.6 on $\partial \widetilde{\Omega}$, it is easy to obtain that

$$
\nu_{\ell} \wedge(\nabla \wedge \mathbf{E})+\widetilde{\boldsymbol{\eta}}\left(\nu_{\ell} \wedge \mathbf{E}\right) \wedge \nu_{\ell}=\nu_{\ell} \wedge(\nabla \wedge \widetilde{\mathbf{E}})+\widetilde{\boldsymbol{\eta}}\left(\nu_{\ell} \wedge \widetilde{\mathbf{E}}\right) \wedge \nu_{\ell}=\mathbf{0} \text { on } \widetilde{\Pi}_{\ell}
$$

We consider the following two separate cases, depending on the values of $\widetilde{\boldsymbol{\eta}}$ on $\widetilde{\Pi}_{\ell}$ associated with the edge-corner $\mathcal{E}\left(\widetilde{\Pi}_{1}, \widetilde{\Pi}_{2}, \boldsymbol{l}\right)$

Case 1. $\left.\widetilde{\boldsymbol{\eta}}\right|_{\widetilde{\Pi}_{\ell}}=0$ or $\left.\widetilde{\boldsymbol{\eta}}\right|_{\widetilde{\Pi}_{\ell}}=\infty, \ell=1,2$. We only consider the case $\left.\widetilde{\boldsymbol{\eta}}\right|_{\widetilde{\Pi}_{\ell}}=\infty$ and the other case can be treated in a similar manner. First, we note that one has from (6.12),

$$
\left(\nu_{\ell} \wedge \mathbf{E}\right) \wedge \nu_{\ell}=0 \quad \text { on } \widetilde{\Pi}_{\ell}, \ell=1,2 .
$$


Let $\widehat{\Pi}_{\ell}$ denote the full flat extension of $\widetilde{\Pi}_{\ell}$ within $\mathbb{R}^{3} \backslash \bar{\Omega}$. We claim that at least one of $\widehat{\Pi}_{\ell}$ is bounded. In fact, if on the contrary that both $\widehat{\Pi}_{1}$ and $\widehat{\Pi}_{2}$ are unbounded, then one has from analytic continuation (noting that $\mathbf{E}$ is real analytic in $\mathbb{R}^{3} \backslash \bar{\Omega}$ ) and $(6.13$ ) that

$$
\lim _{|\mathbf{x}| \rightarrow \infty, \mathbf{x} \in \widehat{\Pi}_{\ell}}\left|\left(\nu_{\ell} \wedge \mathbf{E}\right) \wedge \nu_{\ell}\right|=0, \ell=1,2 .
$$

Using (6.7), we note that $\mathbf{E}^{s}(\mathbf{x}) \rightarrow \mathbf{0}$ as $|\mathbf{x}| \rightarrow \infty$, and hence we further have from (6.14) that

$$
\lim _{|\mathbf{x}| \rightarrow \infty, \mathbf{x} \in \widehat{\Pi}_{\ell}}\left|\left(\nu_{\ell} \wedge \mathbf{E}^{i}\right) \wedge \nu_{\ell}\right|=0, \ell=1,2,
$$

which together with (6.1) readily implies that $\left|\left(\nu_{\ell} \wedge \mathbf{E}\right) \wedge \nu_{\ell}\right|=0, \ell=1,2$. But this is impossible since $\nu_{1}$ and $\nu_{2}$ are linearly independent. Without loss of generality, we can assume that $\widehat{\Pi}_{1}$ is bounded. Clearly, $\widehat{\Pi}_{1}$ and part of $\partial \Omega$ form a bounded domain in $\mathbb{R}^{3} \backslash \bar{\Omega}$, and we denote it as $\Omega_{1}$. It is noted from (6.12) that one has

$$
\nu \wedge(\nabla \wedge \mathbf{E})+\widetilde{\boldsymbol{\eta}}(\nu \wedge \mathbf{E}) \wedge \nu=\mathbf{0} \text { on } \partial \Omega_{1} \backslash \widehat{\Pi}_{1} \text { and } \nu \wedge(\nabla \wedge \mathbf{E})=0 \text { on } \widehat{\Pi}_{1} .
$$

We next show that $\widetilde{\boldsymbol{\eta}}$ can only take 0 or $\infty$ on $\partial \Omega_{1} \backslash \widehat{\Pi}_{1}$. Indeed, we assume on the contrary that there exists a nonempty open subset $\Lambda_{1} \subset \partial \Omega_{1} \backslash \widehat{\Pi}_{1}$ such that $\widetilde{\boldsymbol{\eta}} \in L^{\infty}\left(\Lambda_{1}\right)$ with $\Re(\widetilde{\boldsymbol{\eta}}) \geq 0$ and $\Im(\widetilde{\boldsymbol{\eta}})<0$, and on $\left(\partial \Omega_{1} \backslash \widehat{\Pi}_{1}\right) \backslash \overline{\Lambda_{1}}, \widetilde{\boldsymbol{\eta}}$ takes either 0 or $\infty$. Noting that the Maxwell equations, namely the first two equations in (6.2) are satisfied in $\Omega_{1}$, we have from Green's formula that

$$
\begin{aligned}
\mathbf{i} k \int_{\Omega_{1}}|\mathbf{H}|^{2} & =\int_{\Omega_{1}}(\nabla \wedge \mathbf{E}) \cdot \overline{\mathbf{H}}=\int_{\Omega_{1}} \mathbf{E} \cdot(\nabla \wedge \overline{\mathbf{H}})+\int_{\Omega_{1}}(\overline{\mathbf{H}} \wedge \nu) \cdot \mathbf{E} \\
& =\mathbf{i} k \int_{\Omega_{1}}|\mathbf{E}|^{2}+\int_{\partial \Omega_{1}}(\overline{\mathbf{H}} \wedge \nu) \cdot \mathbf{E}=\mathbf{i} k \int_{\Omega_{1}}|\mathbf{E}|^{2}+\int_{\Lambda_{1}}(\overline{\mathbf{H}} \wedge \nu) \cdot \mathbf{E},
\end{aligned}
$$

where in deriving the last equality, we make use of the fact that $(\overline{\mathbf{H}} \wedge \nu) \cdot \mathbf{E}=0$ on $\partial \Omega_{1} \backslash \overline{\Lambda_{1}}$. Using the fact that $\Im(\widetilde{\boldsymbol{\eta}})<0$ on $\Lambda_{1}$, one can readily infer from 6.17$)$ that $\left.\nu \wedge \mathbf{E}\right|_{\Lambda_{1}}=\mathbf{0}$, which together with (6.16) further implies that $\left.\nu \wedge \mathbf{H}\right|_{\Lambda_{1}}=0$. Hence, by the Holmgren's uniqueness principle (cf. 6]), one has that

$$
\mathbf{E}(\mathbf{x} ; k, \mathbf{d}, \mathbf{p})=\mathbf{0} \text { in } \mathbb{R}^{3} \backslash \bar{\Omega},
$$

which in particular yields that

$$
\lim _{|\mathbf{x}| \rightarrow \infty}|\mathbf{E}(\mathbf{x} ; k, \mathbf{d}, \mathbf{p})|=\mathbf{0} .
$$

But this contradicts to the fact that follows from 6.7):

$$
\lim _{|\mathbf{x}| \rightarrow \infty}|\mathbf{E}(\mathbf{x} ; k, \mathbf{d}, \mathbf{p})|=\lim _{|\mathbf{x}| \rightarrow \infty}\left|\mathbf{p} e^{\mathbf{i} k \mathbf{x} \cdot \mathbf{d}}+\mathbf{E}^{s}(\mathbf{x} ; k, \mathbf{d}, \mathbf{p})\right|=|\mathbf{p}| \neq 0 .
$$

Hence, we actually can find a polyhedral domain $\Omega_{1} \subset \mathbb{R}^{3} \backslash \Omega$ such that one has on $\partial \Omega_{1}$, either $\nu \wedge \mathbf{E}=0$ or $\nu \wedge \mathbf{H}=0$. The situation is reduced to that was considered in [11] and [14]. It is noted that in [14], two far-field patterns are used to handle the above situation. However, the pair of incident fields $\left(\mathbf{E}^{i}, \mathbf{H}^{i}\right)$ in (6.1) in our current case is chosen slightly different from that in [14, which enables one to apply the path-argument from [11 to arrive at a contradiction by starting from $\Omega_{1}$.

Case 2. $\left.\widetilde{\boldsymbol{\eta}}\right|_{\widetilde{\Pi}_{\ell}} \in \mathcal{A}(\boldsymbol{l}), \ell=1,2$; or one of $\left.\widetilde{\boldsymbol{\eta}}\right|_{\widetilde{\Pi}_{\ell}}$ belongs to $\mathcal{A}(\boldsymbol{l})$, and the other one takes 0 or $\infty$; or one of $\left.\widetilde{\boldsymbol{\eta}}\right|_{\widetilde{\Pi}_{\ell}}$ is 0 and the other one is $\infty$. This falls exactly to the situation considered in Theorem 5.2. By the irrationality of the edge-corner as well as the strong uniqueness continuation principle in Theorem 5.2, we readily (6.18), which again leads to the contradiction 6.20 . 
It remains to prove $(6.10)$, and we establish it by contradiction. Let $\Gamma \subset \partial \Omega \cap \partial \widetilde{\Omega} \cap \partial \mathbf{G}$ be an open subset such that $\boldsymbol{\eta} \neq \widetilde{\boldsymbol{\eta}}$ on $\Gamma$. By taking a smaller subset of $\Gamma$ if necessary, we can assume that $\eta$ (respectively $\widetilde{\eta}$ ) is either $L^{\infty}$ or 0 or $\infty$ on $\Gamma$. Clearly, one has $\mathbf{E}=\widetilde{\mathbf{E}}$ in $\mathbf{G}$. Hence it holds that

$$
(\nu \wedge \mathbf{E}) \wedge \nu=(\nu \wedge \widetilde{\mathbf{E}}) \wedge \nu \text { and } \nu \wedge(\nabla \wedge \mathbf{E})=\nu \wedge(\nabla \wedge \widetilde{\mathbf{E}}) \text { on } \Gamma .
$$

and

$$
\nu \wedge(\nabla \wedge \mathbf{E})+\boldsymbol{\eta}(\nu \wedge \mathbf{E}) \wedge \nu=\mathbf{0}, \quad \nu \wedge(\nabla \wedge \widetilde{\mathbf{E}})+\widetilde{\boldsymbol{\eta}}(\nu \wedge \widetilde{\mathbf{E}}) \wedge \nu=\mathbf{0} \quad \text { on } \Gamma .
$$

Combining with the assumption that $\boldsymbol{\eta} \neq \widetilde{\boldsymbol{\eta}}$ on $\mathcal{E}$, we can directly deduce that

$$
\nu \wedge \mathbf{E}=\nu \wedge \mathbf{H}=0 \quad \text { on } \Gamma
$$

which in turn yields by the Holmgren's uniqueness principle (cf. [6]) that $\mathbf{E}=\mathbf{0}$ in $\mathbb{R}^{3} \backslash \Omega$. Therefore, we arrive at the same contradiction as that in 6.19$)$ and $(6.20)$, which readily proves 6.10 ).

The proof is complete.

It is recalled that the convex hull of $\Omega$, denoted by $\mathcal{C H}(\Omega)$, is the smallest convex set that contains $\Omega$. As a direct consequence of Theorem 6.4, we next show that the convex hull of a complex irrational obstacle can be uniquely determined by one farfield measurement. Furthermore, the boundary impedance parameter $\eta$ can be partially identified as well. In fact we have

Corollary 6.5. Consider a fixed triplet of $k \in \mathbb{R}_{+}, \mathbf{d} \in \mathbb{S}^{2}$ and $\mathbf{p} \in \mathbb{R}^{3} \backslash\{\mathbf{0}\}$. Let $(\Omega, \eta)$ and $(\widetilde{\Omega}, \widetilde{\eta})$ be two admissible complex irrational obstacles, with $\mathbf{E}_{\infty}$ and $\widetilde{\mathbf{E}}_{\infty}$ being their corresponding far-field patterns. If $\mathbf{E}_{\infty}$ and $\widetilde{\mathbf{E}}_{\infty}$ satisfy $(6.9)$, then one has that

$$
\mathcal{C H}(\Omega)=\mathcal{C H}(\widetilde{\Omega}):=\Sigma,
$$

and

$$
\boldsymbol{\eta}=\widetilde{\boldsymbol{\eta}} \text { on } \partial \Omega \cap \partial \widetilde{\Omega} \cap \partial \Sigma .
$$

Corollary 6.5 implies that if the underlying polyhedral obstacle is convex, then one can uniquely determine the obstacle as well as its boundary impedance by a single far-field pattern. As a further application of the UCP results established in this work, we consider the unique determination of a rather general class of non-convex obstacles. To that end, we first introduce the aforesaid class of non-convex obstacles.

In the sequel, we denote by $\boldsymbol{P}_{S}(\mathbf{x})$ the projection of a point $\mathbf{x} \in \mathbb{R}^{3}$ onto a set $S$. Let $\partial(\mathcal{C H}(\Omega))=\left\{\Sigma_{\ell} \mid \ell=1, \ldots, N\right\}$, where $\Sigma_{\ell}, \ell=1, \ldots, N$ are the finitely many faces of $\mathcal{C H}(\Omega)$. Let $\mathcal{V}(\Omega)$ and $\mathcal{V}(\mathcal{C H}(\Omega))$ denote, respectively, the sets of vertices of $\Omega$ and $\mathcal{C H}(\Omega)$. It is known that $\mathcal{V}(\mathcal{C H}(\Omega)) \subset \mathcal{V}(\Omega)$. For any vertex $\mathbf{v} \in \mathcal{V}(\Omega) \backslash \mathcal{V}(\mathcal{C H}(\Omega)$ ), we consider the projection, $\boldsymbol{P}_{\Sigma_{j}}(\mathbf{v})$, where $\Sigma_{j} \subset \partial(\mathcal{C H}(\Omega))$ is a face. It is assumed that there exists at least one $\Sigma_{j}$ such that $\mathbf{v}-\boldsymbol{P}_{\Sigma_{j}}(\mathbf{v}) \subset \mathbb{R}^{3} \backslash \Omega$. Then for a face $\Sigma_{l} \subset \partial(\mathcal{C H}(\Omega))$ we say that $\mathbf{v} \vdash \Sigma_{l}$ if

$$
\mathbf{v}-\boldsymbol{P}_{\Sigma_{\ell}}(\mathbf{v})=\underset{\mathbf{v}-\boldsymbol{P}_{\Sigma_{j}}(\mathbf{v}) \in \mathbb{R}^{3} \backslash \Omega, \forall \Sigma_{j} \subset \partial(\mathcal{C H}(\Omega))}{\arg \min }\left|\mathbf{v}-\boldsymbol{P}_{\Sigma_{j}}(\mathbf{v})\right| .
$$

Definition 6.6. Let $\Omega$ be an admissible polyhedral obstacle, and let $\Sigma_{l}$ be a given face of $\partial(\mathcal{C H}(\Omega))$, and $\mathcal{V}_{\mathcal{C}}$ be a given set of finitely many, discrete and distinct points on $\Sigma_{l} . \Omega$ is said to be uniformly concave with respect to $\mathcal{V}_{\mathcal{C}}$ if $\forall \mathbf{v} \in \mathcal{V}(\Omega) \backslash \mathcal{V}(\mathcal{C H}(\Omega)), \mathbf{v} \vdash \Sigma_{l}$ and

$$
\left\{\boldsymbol{P}_{\Sigma_{l}}(\mathbf{v}) \mid \mathbf{v} \in \mathcal{V}(\Omega) \backslash \mathcal{V}(\mathcal{C H}(\Omega))\right\}=\mathcal{V}_{\mathcal{C}} .
$$




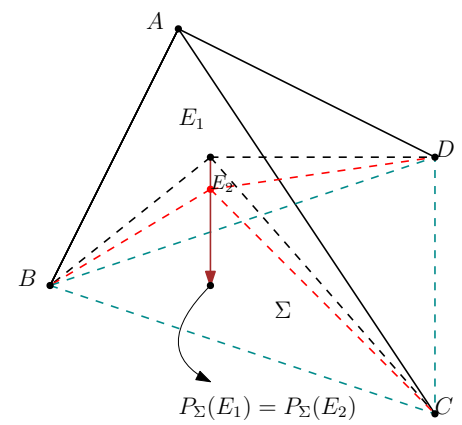

FiguRE 2. Schematic illustration of two different uniformly concave hexahedrons $A B C D E_{1}$ and $A B C D E_{2}$ with $\mathcal{C H}\left(A B C D E_{1}\right)=\mathcal{C H}\left(A B C D E_{2}\right)=$ $A B C D$.

As a simple illustrating example of Definition 6.6, we consider two different uniformly concave hexahedrons $\Omega_{1}:=A B C D E_{1}$ and $\Omega_{2}:=A B C D E_{2}$ that are shown in Figure 2. It is easy to see that $\Omega_{1}$ and $\Omega_{2}$ has the same convex hull, which is the tetrahedron $A B C D$. The vertexes $E_{1}$ and $E_{2}$ corresponding to $\Omega_{1}$ and $\Omega_{2}$ have the same projecting point on the face $\Sigma:=B C D$ of the convex hull $A B C D$. It is pointed out that the vertex corner $\mathcal{V}\left(B E_{2} C, C E_{2} D, B E_{2} D, E_{2}\right) \in \partial \mathbf{G}$, where $B E_{2} C, C E_{2} D, B E_{2} D$ are faces of $\Omega_{2}$ and $\mathbf{G}=\mathbb{R}^{3} \backslash\left(\Omega_{1} \cup \Omega_{2}\right)$.

Theorem 6.7. Consider a fixed triplet of $k \in \mathbb{R}_{+}, \mathbf{d} \in \mathbb{S}^{2}$ and $\mathbf{p} \in \mathbb{R}^{3} \backslash\{\mathbf{0}\}$. Let $(\Omega, \eta)$ and $(\widetilde{\Omega}, \widetilde{\eta})$ be two uniformly concave irrational admissible polyhedral obstacles with respect to the set $\mathcal{V}_{\mathcal{C}}$, with $\mathbf{E}_{\infty}$ and $\widetilde{\mathbf{E}}_{\infty}$ being their corresponding far-field patterns. If $\mathbf{E}_{\infty}$ and $\widetilde{\mathbf{E}}_{\infty}$ satisfy 6.9 , then

$$
\Omega=\widetilde{\Omega} \text { and } \boldsymbol{\eta}=\widetilde{\boldsymbol{\eta}} .
$$

Proof. We prove this theorem by contradiction. Assume that $\Omega \neq \widetilde{\Omega}$ but $(6.9)$ is still fulfilled. From Corollary 6.5 , we have $\mathcal{C H}(\Omega)=\mathcal{C H}(\widetilde{\Omega})$, which implies that the vertices of $\Omega$ contributing to $\mathcal{C H}(\Omega)$ are the same as the corresponding vertices of $\widetilde{\Omega}$ contributing to $\mathcal{C H}(\widetilde{\Omega})$. We shall prove that there must exit an edge-corner $\mathcal{E}\left(\Pi_{1}, \Pi_{2}, \mathbf{x}_{c}\right) \in \partial \mathbf{G}$, where $\mathbf{G}$ is the unbounded connected component of $\mathbb{R}^{3} \backslash \overline{(\Omega \cup \widetilde{\Omega})}$. Since $\Omega \neq \widetilde{\Omega}$, there exits an edge $\boldsymbol{l} \subset \partial \Omega \backslash \partial \widetilde{\Omega}$ or $\boldsymbol{l} \subset \partial \widetilde{\Omega} \backslash \partial \Omega$. Without loss of generality, we assume that $\boldsymbol{l} \subset \partial \widetilde{\Omega} \backslash \partial \Omega$. In the sequel, we let $\mathbf{a}_{\boldsymbol{l}}$ and $\mathbf{b}_{\boldsymbol{l}}$ denote the two vertices of the line segment $\boldsymbol{l}$. We divide our remaining proof into two separate cases.

Case 1. Suppose that $\mathbf{a}_{\boldsymbol{l}} \in \mathcal{V}(\mathcal{C H}(\widetilde{\Omega}))$ and $\mathbf{b}_{\boldsymbol{l}} \in \mathcal{V}(\mathcal{C H}(\widetilde{\Omega}))$. Therefore, $\boldsymbol{l} \subset \partial \mathbf{G} \cap \partial \widetilde{\Omega}$. There exits a point $\mathbf{x}_{c} \in \boldsymbol{l}$ and a sufficient small $h \in \mathbb{R}_{+}$such that

$$
B_{h}\left(\mathbf{x}_{c}\right) \cap \partial \widetilde{\Omega}=\widetilde{\Pi}_{\ell}, \quad \ell=1,2,
$$

where $\widetilde{\Pi}_{\ell}$ are two flat subsets lying on the faces of $\widetilde{\Omega}$ that intersect at $\mathbf{x}_{c}$. Clearly, $\mathbf{x}_{c} \in \boldsymbol{l}$ is an edge-corner point.

Case 2. Suppose that there exits at least one of $\mathbf{a}_{\boldsymbol{l}}$ and $\mathbf{b}_{\boldsymbol{l}}$ belonging to $\mathcal{V}(\widetilde{\Omega}) \backslash \mathcal{V}(\mathcal{C H}(\widetilde{\Omega}))$; namely, $\mathbf{x}_{c} \in \mathcal{V}(\widetilde{\Omega}) \backslash \mathcal{V}(\mathcal{C H}(\widetilde{\Omega}))$, where $\mathbf{x}_{c}$ could be either $\mathbf{a}_{\boldsymbol{l}}$ or $\mathbf{b}_{\boldsymbol{l}}$. Since $\Omega$ and $\widetilde{\Omega}$ are uniformly concave admissible polyhedral obstacles with respect to the set $\mathcal{V}_{\mathcal{C}}$, there exits a face $\Sigma_{\ell} \Subset \partial(\mathcal{C H}(\Omega))$ such that $\mathbf{x}_{c} \vdash \Sigma_{\ell}$ and $\mathcal{V}_{\mathcal{C}} \Subset \Sigma_{\ell}$. Furthermore, we know that there exits a vertex $\mathbf{x}_{c, \Omega} \in \mathcal{V}(\Omega) \backslash \mathcal{V}(\mathcal{C H}(\Omega)$ such that

$$
\mathbf{x}_{c, \Omega} \vdash \Sigma_{\ell}, \quad \boldsymbol{P}_{\Sigma_{\ell}}\left(\mathbf{x}_{c, \Omega}\right)=\boldsymbol{P}_{\Sigma_{\ell}}\left(\mathbf{x}_{c}\right) \in \mathcal{V}_{\mathcal{C}} .
$$


Since $\mathbf{x}_{c, \Omega}$ and $\mathbf{x}_{c}$ are distinct, it holds that

$$
\mathrm{d}\left(\mathbf{x}_{c}, \Sigma_{\ell}\right) \neq \mathrm{d}\left(\mathbf{x}_{c, \Omega}, \Sigma_{\ell}\right),
$$

where $\mathrm{d}\left(\mathbf{x}_{c}, \Sigma_{\ell}\right)$ is the distance between $\mathbf{x}_{c}$ and $\Sigma_{\ell}$. Without loss of generality, we may assume that $\mathrm{d}\left(\mathbf{x}_{c}, \Sigma_{\ell}\right)<\mathrm{d}\left(\mathbf{x}_{c, \Omega}, \Sigma_{\ell}\right)$. Hence, one can conclude that

$$
\mathbf{x}_{c} \in \partial \mathbf{G},
$$

which also indicates that $\mathbf{x}_{c}$ lies outside $\Omega$. Let $h \in \mathbb{R}_{+}$be sufficiently small such that $B_{h}\left(\mathbf{x}_{c}\right) \Subset \mathbb{R}^{2} \backslash \bar{\Omega}$, then due to the fact that $\mathcal{V}_{\mathcal{C}}$ is discrete and distinct we can conclude that

$$
B_{h}\left(\mathbf{x}_{c}\right) \cap \partial \widetilde{\Omega}=\widetilde{\Pi}_{\ell}, \quad \ell=1,2,
$$

where $\widetilde{\Pi}_{\ell}$ are two plane cells lying on the faces of $\widetilde{\Omega}$ that intersect at $\mathbf{x}_{c}$.

The remaining proof is similar to the that of Theorem 6.4, which is omitted.

Finally, we remark that in this section, we only consider the case that the underlying obstacle is irrational in order to make use of the strong unique continuation principle in Theorem 5.2. That is, in the contradiction argument in proving Theorems 6.4 and 6.7, one can find an edge-corner that can lead to the vanishing of the total wave field outside the obstacle by the strong unique continuation principle in Theorem 5.2 . However, we would like to emphasize that the same argument would work for the case that the underlying obstacle is of a general polyhedral shape, subject to a some slight modification. In fact, in such a case, it may happen that the edge-corner in the contradiction argument is rational, and hence instead of Theorem 5.2, one would need to make use of the finite vanishing order results in Theorems 3.6, 4.2, 4.4 and 4.5 to obtain that the total wave field is "small" around the edge-corner (compared to the totally vanishing in the irrational case). Hence, a contradiction can be obtained if one requires that the total wave field outside the obstacle is everywhere "big", which can be fulfilled in certain scenarios of practical interest, see e.g. [4]. Nevertheless, we shall not explore this direction any further in this paper.

6.2. Information-encoding for inverse problems and generalised Holmgren's uniqueness principle. We recall the classical Holmgren's theorem for an elliptic PDO $\mathcal{P}$ with real-analytic coefficients (cf. [18]). If $\mathcal{P} \mathbf{u}$ is real analytic in a connected open neighbourhood of $\Omega$, then $\mathbf{u}$ is also real-analytic. The Holmgren's theorem applied to $\mathbf{u}=(\mathbf{E}, \mathbf{H})$ in $(1.1)$, we immediately see that $(\mathbf{E}, \mathbf{H})$ is real-analytic in $\Omega$. Let $\Gamma$ be an analytic surface in $\Omega$. Suppose that

$$
\nu \wedge \mathbf{E}=\mathbf{0} \quad \text { and } \quad \nu \wedge \mathbf{H}=0 \quad \text { on } \Gamma
$$

then by the Cauchy-Kowalevski theorem, one readily has that $\mathbf{E}=\mathbf{H} \equiv 0$ in $\Omega$. This is known as the Holmgren's uniqueness principle. In fact, in the proofs of Theorems 6.4 and 6.7. we have made use of the Holmgren's principle in the case that $\Gamma$ is an open subset of a plane. In the sequel, to ease the exposition and with a bit abuse of notations, we simply refer to $\Gamma$ as a plane in such a case, though it may actually be an open subset of a plane. Our results established in Theorems 3.6, 4.2, 4.4, 4.5 and 5.2 can be regarded as generalizing the Holmgren's uniqueness principle as discussed in what follows.

Suppose that there are two planes $\widetilde{\Pi}_{1}$ and $\widetilde{\Pi}_{2}$ which intersect at a line segment $\boldsymbol{l}$ within $\Omega$ (see Fig. 1), and

$$
\nu \wedge \mathbf{E}=\mathbf{0} \text { on } \widetilde{\Pi}_{1} \quad \text { and } \quad \nu \wedge \mathbf{H}=\mathbf{0} \text { on } \widetilde{\Pi}_{2} .
$$

Let $\angle\left(\widetilde{\Pi}_{1}, \widetilde{\Pi}_{2}\right)=\alpha \pi$. Suppose that $\alpha=1 / N$ with $N \in \mathbb{N}$. Then according to Theorem 4.2 , we know that the vanishing order of $\mathbf{E}$ around $\boldsymbol{l}$ is at least $N$. Letting $N \rightarrow \infty$, we see that in the limiting case, one has 6.24 with $\widetilde{\Pi}_{1}=\widetilde{\Pi}_{2}=\Gamma$ as well as that the vanishing order becomes infinity. That is, the classical Holmgren's uniqueness principle associated a plane $\Gamma$ for the Maxwell system (1.1) is the limiting case of our result in 
Theorem 4.2. It is surprisingly interesting that we have generalised such an observation in three aspect. First, the angle between the two intersecting planes is not infinitesimal and hence the vanishing order may be finite. Second, if the angle is irrational, not necessarily infinitesimal, the vanishing order is still infinity. Third, the homogeneous condition on the plan can be the much more general impedance condition.

The application to inverse problem of the above observation can be described as follows. In inverse problems with electromagnetic probing, one usually sends a pair of incident fields and then collects the corresponding scattered wave data away from the inhomogeneous object; see (6.8) associated with 6.2. In the following, we first take (6.8) as a specific exam elucidate the basic idea. Usually, the collection of the data is made on an analytic surface, say $\Gamma$, in the form $\left(\left.\nu \wedge \mathbf{E}\right|_{\Gamma},\left.\nu \wedge \mathbf{H}\right|_{\Gamma}\right)$. Then by the Holmgren's principle, we know that the information encoded into $\left(\left.\nu \wedge \mathbf{E}\right|_{\Gamma},\left.\nu \wedge \mathbf{H}\right|_{\Gamma}\right)$ is equivalent to knowing the electromagnetic fields outside the scattering obstacle, namely $\mathbb{R}^{3} \backslash \bar{\Omega}$, and hence is equivalent to the far-field pattern $\mathbf{E}_{\infty} / \mathbf{H}_{\infty}$. According to Theorem 5.2 , the measurement data can also be collected as $\left(\nu \wedge \mathbf{H}+\left.\boldsymbol{\eta}_{1} \nu \wedge \mathbf{E}\right|_{\widetilde{\Pi}_{1}}, \nu \wedge \mathbf{H}+\left.\boldsymbol{\eta}_{2} \nu \wedge \mathbf{H}\right|_{\widetilde{\Pi}_{2}}\right)$ as long as $\widetilde{\Pi}_{1}$ and $\widetilde{\Pi}_{2}$ can intersect within $\mathbb{R}^{3} \backslash \bar{\Omega}$ with an irrational angle. Clearly, due to the analytic extension, it is not necessary for $\widetilde{\Pi}_{1}$ and $\widetilde{\Pi}_{2}$ to really intersect each other. The irrational intersection seems to be too restrictive and one can relax it to be a rational intersection with a large degree. Clearly, this conceptual information encoding technique also work for the other inverse electromagnetic scattering problem where the underlying object is not necessary an impenetrable obstacle as that considered in (6.8). We hope that it might find practical applications in some special situations.

\section{REFERENCES}

[1] M. Abramowitz and I. A. Stegun, Handbook of mathematical functions: with formulas, graphs, and mathematical tables, vol. 55, Courier Corporation, 1964.

[2] L. Alessandra and V. Felli, Unique continuation from the edge of a crack, arXiv:2004.11177

[3] G. Bao, H. Liu and J. Zou, Nearly cloaking the full Maxwell equations: cloaking active contents with general conducting layers, J. Math. Pures Appl. (9), 101 (2014), 716-733.

[4] X. Cao, H. Diao and H. Liu, Determining a piecewise conductive medium body by a single far-field measurement, arXiv:2005.04420

[5] X. Cao, H. Diao, H. Liu and J. Zou, On nodal and generalized singular structures of Laplacian eigenfunctions and applications to inverse scattering problems, arXiv:1902.05798, 2019.

[6] D. Colton and R. Kress, Inverse Acoustic and Electromagnetic Scattering Theory, 3rd edition, Springer-Verlag, Berlin, 2013.

[7] D. Colton and R. Kress, Looking back on inverse scattering theory, SIAM Review, 60 (2018), no. 40, 779-807.

[8] M. Costabel, M. Dauge and R. Duduchava, Asymptotics without logarithmic terms for crack problems, Comm. PDEs, 28 (2003), 869-926.

[9] G. Dal Maso, G. Orlando and R. Toader, Laplace equation in a domain with a rectilinear crack: higher order derivatives of the energy with respect to the crack length, Nonlinear Differential Equations Appl., 22 (2015), 449-476.

[10] S. G. Krantz and H. R. Parks, A primer of real analytic functions, 2nd edition, Birkhäuser Boston, Inc., Boston, MA, 2002.

[11] H. Liu, A global uniqueness for formally determined inverse electromagnetic obstacle scattering, Inverse Problems, 24 (2008), 035018 (13pp).

[12] H. Liu, L. Rondi and J. Xiao, Mosco convergence for $H($ curl $)$ spaces, higher integrability for Maxwell's equations, and stability in direct and inverse EM scattering problems, J. Eur. Math. Soc. (JEMS), 21 (2019), 2945-2993.

[13] H. Liu, M. Yamamoto and J. Zou, Reflection principle for the Maxwell equations and its application to inverse electromagnetic scattering, Inverse Problems, 23 (2007), 2357-2366.

[14] H. Liu, M. Yamamoto and J. Zou, New reflection principles for Maxwell's equations and their applications, Numer. Math. Theor. Meth. Appl., 2 (2009), No. 1, 1-17.

[15] H. Liu and T. Zhou, On approximate electromagnetic cloaking by transformation media, SIAM J. Appl. Math., 71 (2011), 218-241. 
[16] H. Liu and J. Zou, Uniqueness in an inverse acoustic obstacle scattering problem for both sound-hard and sound-soft polyhedral scatterers, Inverse Problems, 22 (2006), 515-524.

[17] J.-C. Nédélec, Acoustic and Electromagnetic Equations, Springer-Verlag, New York, 2001.

[18] F. Treves, Introduction to Pseudodifferential and Fourier Integral Operators, Vol. 1, Plenum Press, New York, 1980.

School of Mathematics and Statistics, Northeast Normal University, Changchun, Jilin 130024, CHINA.

E-mail address: hadiao@nenu.edu.cn

Department of Mathematics, City University of Hong Kong, Kowloon, Hong Kong, CHINA.

E-mail address: hongyu.liuip@gmail.com, hongyliu@cityu.edu.hk

School of Mathematics and Statistics, Northeast Normal University, Changchun, Jilin 130024, CHINA.

E-mail address: $907278586 @ q q . c o m$

Department of Mathematics, The Chinese University of Hong Kong, Hong Kong, China

E-mail address: zou@math.cuhk.edu.hk 